University of Redlands

\title{
Aerodrome Obstruction Identification Surfaces
}

\author{
A Major Individual Project submitted in partial satisfaction of the requirements \\ for the degree of Master of Science in Geographic Information Systems \\ by \\ Teran Chun Fung Wong \\ Douglas Flewelling, Ph.D., Committee Chair \\ Mark P. Kumler, Ph.D.
}

December 2009 
Aerodrome Obstruction Identification Surfaces

Copyright (C) 2009

by

Teran Chun Fung Wong 
The report of Teran Chun Fung Wong is approved.

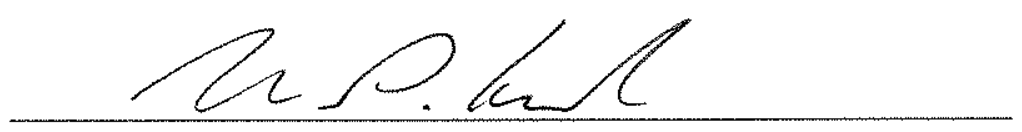

Mark Kumler, Ph.D.

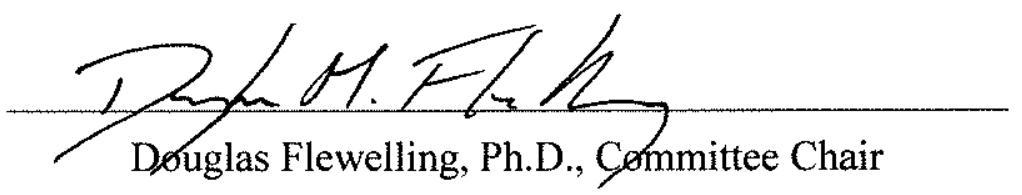

December 2009 



\section{Acknowledgements}

I would like to take this opportunity to thank all the people who helped me through this major individual project. The successful completion of this project would not have been possible without the support and encouragement of these individuals.

Special thanks to Shane Barrett who gave me inspiration in choosing the project idea and supporting me with all the ESRI resources.

Sincere thanks to my program advisor Douglas Flewelling for his calm and constant guidance.

I would like to thank my lovely wife for her unconditional support and encouragement. Without her support, I would not have finished this project. 



\author{
Abstract \\ Aerodrome Obstruction Identification Surfaces \\ by \\ Teran Chun Fung Wong
}

This project presents a custom-built GIS solution to assist in the production of obstruction identification surfaces (OIS) for the world's airports. OIS is one of the key elements in providing a safe environment for air travel as many airports process a huge number of flight operations every day. The Federal Aviation Administration (FAA) and the International Civil Aviation Organization (ICAO) have put great effort into improving world air safety. FAA published Federal Aviation Regulations (FAR) and ICAO published Annexes. These regulations and annexes govern all aviation activities in order to improve the safety of air travel. In particular, a complete OIS specification is described in both regulations and annexes.

The project client, ESRI Aeronautical Solution, sought a custom-built GIS solution for users to generate the OIS in an effective way and to plan for further obstacle analysis. A file geodatabase was developed to both manage the aeronautical data and store the generated OIS feature data. A geoprocessing tool was developed to generate the OIS combined with the required aeronautical data and OIS specifications from FAA and ICAO. With the help of this innovative GIS solution, users can easily generate the OIS in seconds and maintain them in a more globalized and generalized format, thereby supporting their preliminary efforts towards creating a safer air travel environment. 


\section{Table of Contents}

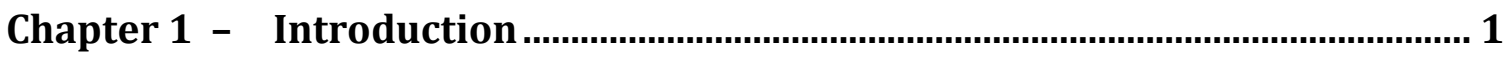

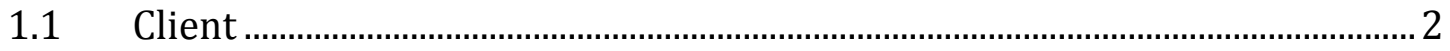

$1.2 \quad$ Problem Statement ..............................................................................................

$1.3 \quad$ Proposed Solution .................................................................................................. 4

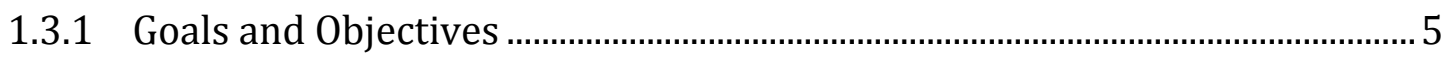

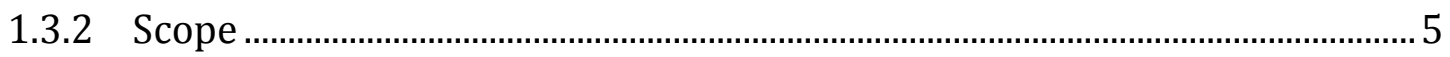

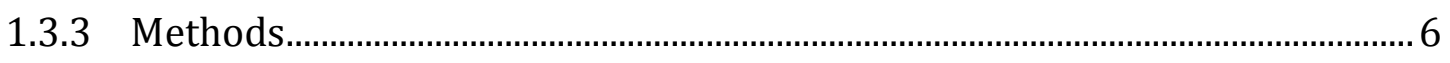

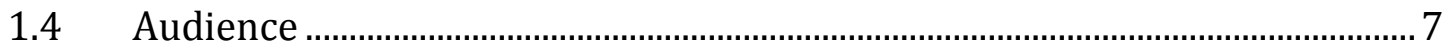

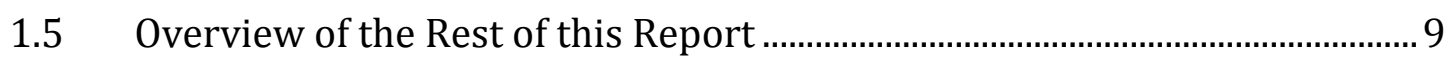

Chapter 2 - Background and Literature Review...............................................11

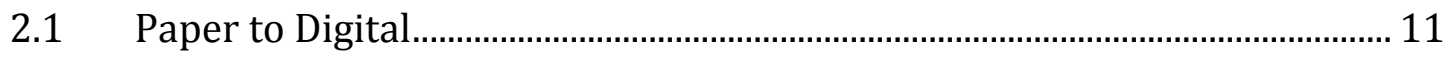

2.2 Obstruction Identification Surfaces ................................................................. 12

2.2.1 Obstruction Identification Surfaces Specification from the FAA ................. 13

2.2.2 Obstruction Identification Surfaces Specification from ICAO ...................... 15

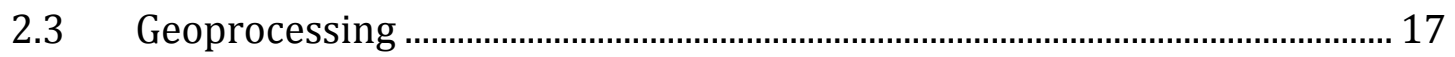

2.4 Past Research.................................................................................................... 18

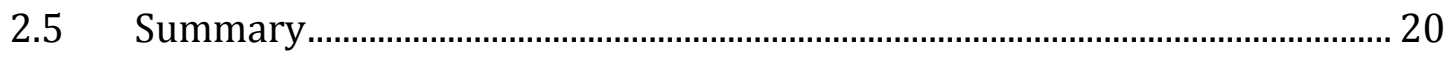

Chapter 3 - Systems Analysis and Design …...................................................21

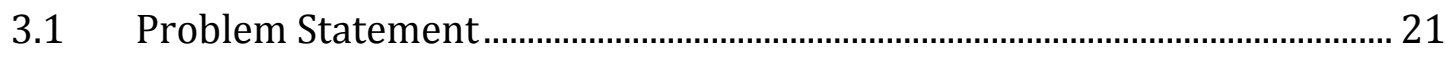

3.2 Requirements Analysis ................................................................................ 22

3.2.1 Functional Requirements.............................................................................. 22 


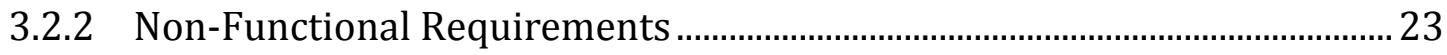

3.3 System Design ............................................................................................ 26

3.4 Project Plan .................................................................................................... 28

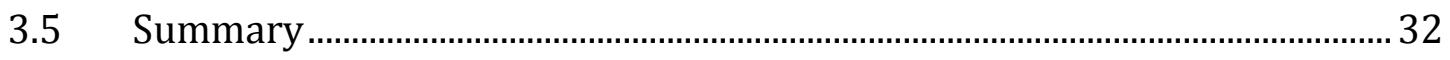

Chapter 4 - Database Design ..........................................................................33

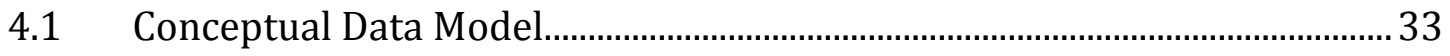

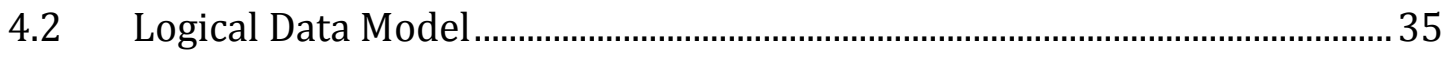

4.3 Physical Data Model.................................................................................. 37

$4.4 \quad$ Data Sources .................................................................................................... 41

$4.5 \quad$ Data Scrubbing and Loading ……………………......................................... 42

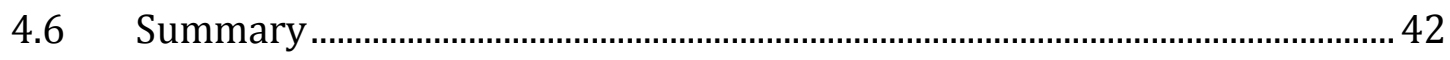

Chapter 5 - Geoprocessing Tool Design ..............................................................44

5.1 Geoprocessing Components ...................................................................... 44

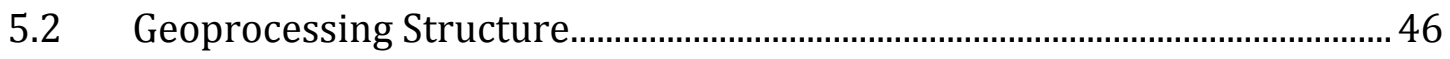

5.3 OIS Parameters and Decision Structure …………………………………….. 48

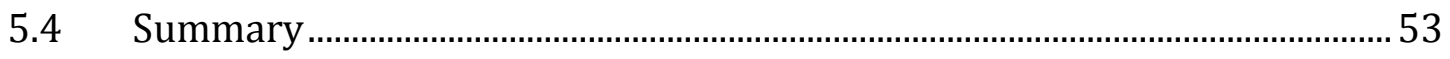

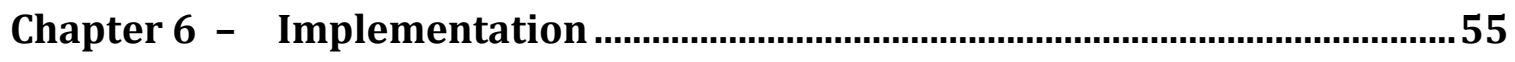

6.1 OIS Geoprocessing Function Factory ……………………………………... 55

6.2 OIS Geoprocessing Function.......................................................................... 56

6.3 OIS Parameters and OIS Creation modules ……………………………........ 61

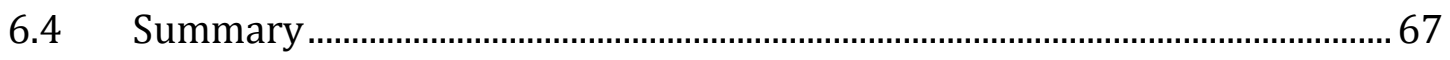

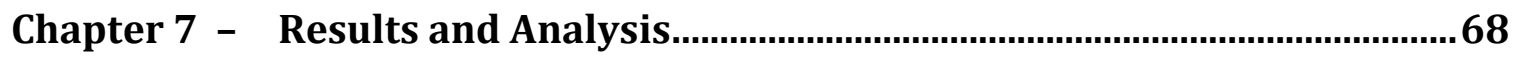


7.1 OIS in Two-Dimensional View....................................................................... 68

7.2 OIS in Three-Dimensional View........................................................................... 73

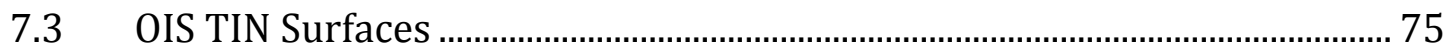

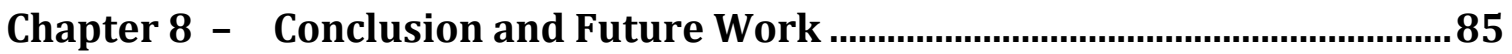

8.1 Conclusion ................................................................................................. 85

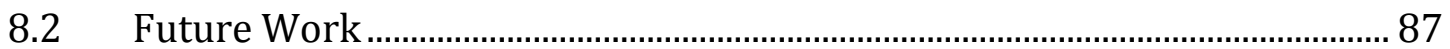

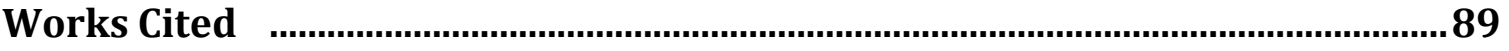

Appendix A. OIS Geoprocessing Tool Source Code ….............................................93 



\section{Table of Figures}

Figure 3: System Functionalities Diagram .........................................................2

Figure 4: Gantt Chart for OIS Project ............................................................28

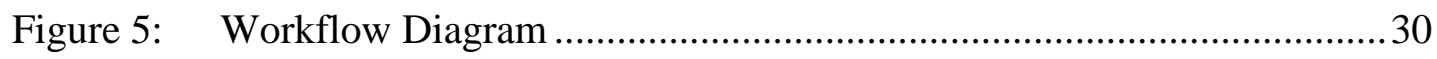

Figure 6: Conceptual Data Model ..........................................................................

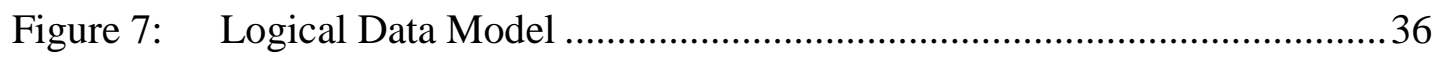

Figure 8: Simplified Aeronautical Database Model For eTOD ..............................41

Figure 9: Geoprocessing Structure …………………………………………..... 46

Figure 10: OIS Geoprocessing Tool Decision Structure …………………….....52

Figure 11: Function Factory Property General Structure ………...........................55

Figure 12: Function Factory Method General Structure …………………….......56

Figure 13: General Structure of OIS Parameter Module ........................................61

Figure 14: $\quad$ Execute Method Function Header ……………………………….....62

Figure 15: Primary Surface Method Function Header ...........................................62

Figure 16: Approach Surface Method Function Header ..........................................63

Figure 17: Horizontal and Conical Surface Method Function Header..................64

Figure 18: Transitional Surface Method Function Header .....................................64

Figure 19: Get Intersection Point Method Function Header .................................65

Figure 20: Construct Surface Arc Method Function Header..................................66

Figure 21: $\quad$ Make Z Aware Method Function Header ............................................66

Figure 22: Unit Conversion Method Function Header..........................................66

Figure 23: $\quad$ FAA OIS in Two-Dimensional View ………......................................69

Figure 24: $\quad$ eTOD OIS in Two-Dimensional View …………………................ 
Figure 25: $\quad$ Number of Surfaces for FAA and eTOD ................................... 71

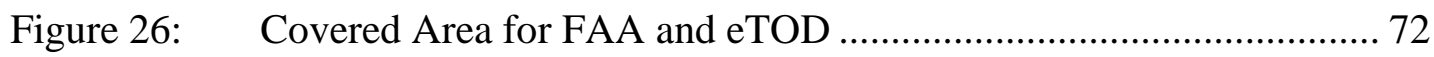

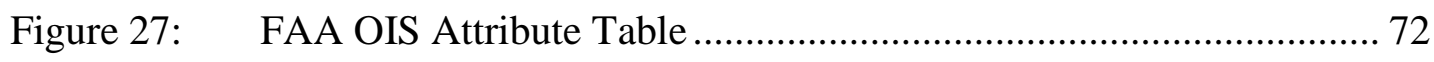

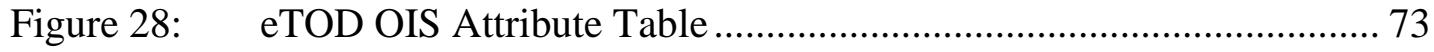

Figure 29: $\quad$ FAA OIS in Three-Dimensional View....................................... 74

Figure 30: $\quad$ eTOD OIS in Three-Dimensional View ..................................... 74

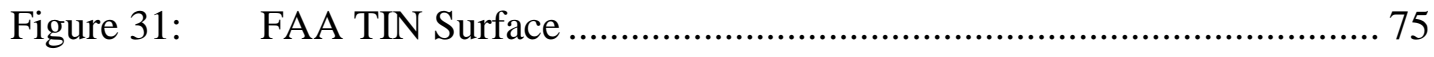

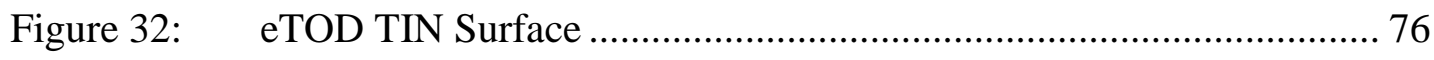

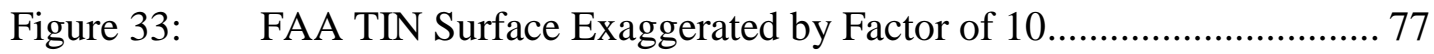

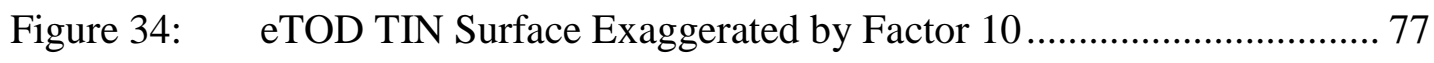

Figure 35: $\quad$ FAA TIN Surface with Node and Edges Symbols ........................... 79

Figure 36: Zoomed FAA TIN Surface with Node and Edge Symbols .............. 79

Figure 37: $\quad$ eTOD TIN Surface with Node and Edge Symbols .......................... 80

Figure 38: Zoomed eTON TIN Surface with Node and Edge Symbols ............. 80

Figure 39: $\quad$ FAA OIS TIN Surface with Aspect Symbol ................................. 81

Figure 40: $\quad$ eTOD OIS TIN Surface with Aspect Symbol ................................ 82

Figure 41: $\quad$ FAA TIN Surface with Elevation Symbol .................................... 83

Figure 42: $\quad$ eTOD TIN Surface with Elevation Symbol ................................... 83 


\section{List of Tables}

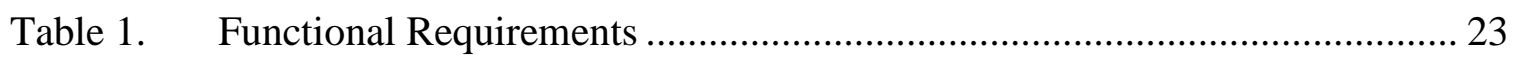

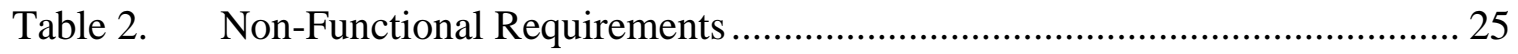

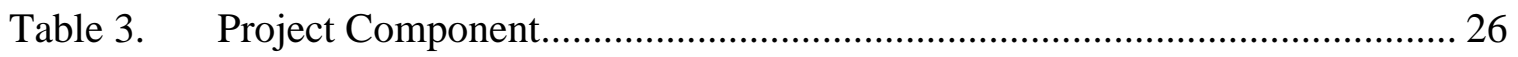

Table 4. Extreme Programming rules and practice ............................................ 31

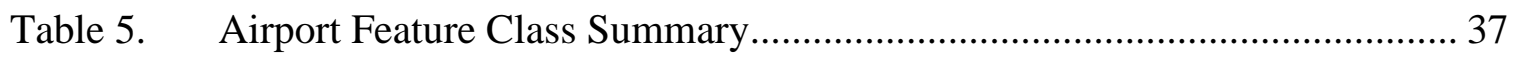

Table 6. Runway Feature Class Summary ........................................................ 38

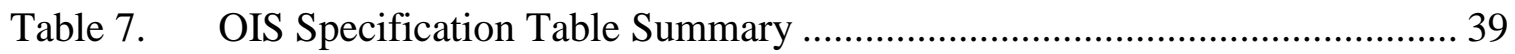

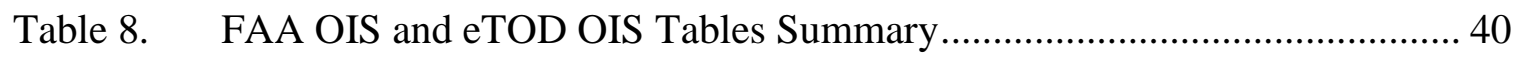

Table 9. GPFunction Properties and Methods .................................................... 47

Table 10. Geoprocessing Function Factory Properties and Methods ......................... 48

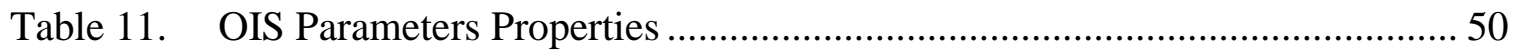

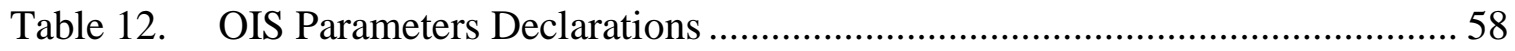

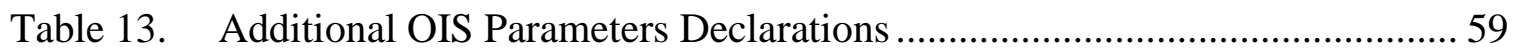





\section{List of Acronyms and Definitions}

\begin{tabular}{ll} 
OIS & Obstruction Identification Surfaces \\
GP & Geoprocessing \\
PLTS & Production Lint Tool Set \\
FAA & Federal Aviation Administration \\
ICAO & International Civil Aviation Organization \\
eTOD & Electronic Terrain and Obstacle Database \\
NGA & National Geospatial-Intelligence Agency \\
XP & Extreme Programming \\
AIS & Aeronautical Information Service \\
RTCA & Radio Technical Committee for Aeronautics \\
EUROCAE & European Organization for Civil Aviation Equipment \\
NOAA & National Oceanic and Atmospheric Administration \\
AOC & Aeronautical Obstruction Chart \\
FATO & Final Approach and Takeoff Area \\
GFID & Global Format Identifier \\
\hline
\end{tabular}





\section{Chapter 1 - Introduction}

The purpose of aerodrome obstruction identification surface project is to develop a prototype application to simplify and improve the obstruction identification surfaces generating process in and around airfields.

Air safety has always been an important issue in air travel. It relies on the efficient use of the airspace in the vicinity of the airport and along the way to the airport. Many accidents or near misses involving aircraft occur near the landing site due to the aeronautical charts being in different formats, the use of outdated aeronautical information, or poor communication due to differing aeronautical languages.

On April $3^{\text {rd }}$, 1996, Air Force CT-43 crashed into a mountainside in Croatia while attempting an instrument approach to Dubrovnik Airport, killing everyone onboard. An Air Force investigation attributed the crash to a poorly designed landing approach (Hughes, 1996).

On November $24^{\text {th }}, 2001$, Crossair Flight LX3597 crashed during its approach to Zurich International Airport. The investigation concluded that the accident was a controlled flight into terrain caused by the captain deliberately descending below the minimum descent altitude without having achieved the required visual contact with either the approach lights or the runway. The range of hills the plane crashed into was also not marked in the Jeppesen approach chart used by the crew (BFU Switzerland, 2004).

On February, 2008, Santa Barbara Airlines Flight 518 from Merida, Venezuela to Caracas crashed, killing 46 people. The investigation report indicated the crew departed Merida with inoperative navigation equipment and subsequently became disoriented in 
the mountainous terrain surrounding the airport, crashing into the side of a mountain while trying to determine their location (Aviation Safety Network, 2008).

One major factor was incomplete or incorrect terrain and obstacle information. The pilots were unaware of the danger until it was too late. Therefore, complete terrain and obstacle data is vital to flight planning, adapting to the unpredictable air travel environment, and emergency landing. In short, better information would improve air safety margins.

In January, 2009, Los Angeles International Airport processed over 40,000 flight operations (Los Angeles International Airport, 2009) and there are many international airports around the world which process even more. The busy air traffic situation has made air safety even more critical and wide ranging due to the ever increasing number of fights. This paper presents an innovative system by utilizing a geographic information system to enhance the aeronautical chart generation and to manage them in an effective way.

\subsection{Client}

The client for this project is ESRI Aeronautical Solution team, which has been engaged in creating and updating aeronautical charts, organizing and manipulating aeronautical data, and providing customized solution to clients in aeronautical domain. The primary contact person of this project is Shane Barrett, Aeronautical Solution production manager.

Mr. Barrett joined the ESRI Aeronautical Solution team in 2007. His role involves the planning and coordination of aeronautical projects. In 2008, the Aeronautical Solution team reacted to their client's needs to develop a custom-built eTOD solution. Building 
upon the success of the eTOD dataset production, ESRI's Aeronautical Solution team will develop solution which symbolizes and represents the data from the eTOD dataset. As an accompaniment to the team's efforts, this project presents a custom-built geoprocessing tool to generate the OIS surface.

In support of the OIS project, ESRI will provide the following,

- ESRI GIS product in version 9.3.1 environment

- Aeronautical database schema and file structure

- Production Line Tool Set (PLTS) file structure

- PLTS coding standard documents

- Federal Aviation Administration (FAA) specification for Obstruction Identification Surface (OIS)

- Electronic Terrain and Obstruction Database (eTOD) specification for OIS

- Project area data with all layers.

\subsection{Problem Statement}

Federal Aviation Administration (FAA) International Civil Aviation Authority (ICAO), a lead aviation agency of the United Nation, has established goals towards modernizing global air traffic control. The first step in ICAO's plan is obtaining and managing digital geospatial data. ESRI's Aeronautical Solution team recognized a great opportunity to assist their current and potential airport clients to prepare the eTOD dataset in the short time allotted by ICAO. Now the Aeronautical Solution team faces the challenge of finding a method to simplify and improve the OIS generating process. In this case, to simplify means having fewer buttons to click for the chart producer when creating the OIS chart and to improve means to decrease the processing time for the OIS generation. 
Currently there are many sources providing obstacle data. However, there is virtually no one with the requisite expertise to advise or help them in the best use of technology for OIS and the relationship between OIS and terrain and obstacle data. OIS determines which objects are vertical obstructions. An object is considered as a vertical obstruction if it penetrates the OIS. With these data, pilots can decide how to best approach or depart from an airfield. In addition, the maximum allowable takeoff weight for a particular runway can also be determined. Currently there is not much computer software available to provide a complete tool to generate the OIS. Without an accurate OIS, information about the obstruction areas will be missing or distorted in and around an airfield. Due to the reasons cited above, an accurate OIS and an effective means of generating up to date OIS will provide the best way to avoid many accidents and near misses involving aircraft near the landing sites.

\subsection{Proposed Solution}

The proposed solution to the problem mentioned in section 1.2 is to use Geoprocessing to simplify and improve the OIS generating process.

The purpose of Geoprocessing is to automate the GIS operations. Geoprocessing has the ability to allow the user to combine a series of Geoprocessing tools, using the output of one Geoprocessing tool as the input for another Geoprocessing tool, in a sequence of operations using models and scripts.

OIS charting involves several components and receives input from many sources, including technology, design, and human. Due to its complexity, this project will concentrate on the development of a Geoprocessing tool to generate the OIS. 
The OIS Geoprocessing tool generates the OIS by providing the airport runway features in point, line or polygon format and the specification of the OIS from eTOD and FAA. The output is a polygon type feature class stored in a Geodatabase.

\subsubsection{Goals and Objectives}

The OIS project is divided into two main parts. The first part of this project is to analyze the OIS specifications from eTOD and FAA. These two sets of specifications have their similarities and differences. The second part of this project will be to develop a custombuilt Geoprocessing tool to generate the OIS.

Accomplishment of this project will produce the safest possible terminal procedures. In addition, it will provide the best use of technology for OIS.

Successful completion of this project may establish a new upfront services contract in FAA, National Geospatial-Intelligence Agency (NGA), and any other governing agencies or organizations that will benefit from the technology and workflows designed under this project.

Currently there is little competition to create database-driven OIS charts with a commercial, off-the-shelf software package. As a result, there is a significant market for OIS capability at airports across the country. Successful completion of this project would be an excellent case study which ESRI could market to potential PLTS aeronautical customers.

\subsubsection{Scope}

ESRI added Electronic Terrain and Obstacle Database (eTOD) capabilities to PLTS for Aeronautical Solution in 2008 (ESRI, 2009). The OIS project is not only a continuation of 
the success of the eTOD; but also represents a new prototype tool on OIS with another set of specifications from FAA.

Different governing agencies have different OIS specifications in terms of zones and surfaces. The OIS project will focus on the interpreting of specifications from eTOD and FAA, analyzing data of the study area, batch processing of aeronautical processing tools that are the Geoprocessing tools for Aeronautical solution, and preparing for the future development.

Salinas Municipal Airport is used for OIS project to generate OIS. A geodatabase will store the generated OIS polygon features. The input data will be loaded into the ArcCatelog and ArcMap and be examined for consolidation.

The OIS project will involve an ESRI-issued laptop and an ESRI-issued desktop for the development of the Geoprocessing tools. Those Geoprocessing tools will be written in $\mathrm{C}++$ or $\mathrm{C} \#$ language and employ an object-oriented programming paradigm.

\subsubsection{Methods}

The methodology for the OIS project will mostly follow the Extreme Programming (XP)method . Extreme Programming leads to the development of a process that is more responsive to customer needs than traditional methods, creating a wholly different and better quality of software.

The following rules and practices of Extreme Programming can be applied and will function well in this project:

\section{- Planning}

O User stories: Meet with ESRI employee at Redlands 
$\bigcirc \quad$ Make frequent small releases: Deploy the prototype OIS Geoprocessing tool to Redlands in order to get valuable feedback in time to have an impact on the system's development, while the OIS specification is still in drafting status.

○ Fix XP when it breaks: Due to the complexity, the project may require adjustment and modification during the production cycle period.

\section{- Designing}

○ Simplicity: Keep the Geoprocessing tool as simple as possible.

- Choose a system metaphor: Inherit this from the ESRI PLTS framework and ESRI Aeronautical Solution

No Functionality is added early: Concentrate on currently scheduled requirements instead of potential future needs or extra flexibility.

- Coding

○ Code must be written to agreed standards: Code will follow the ESRI PLTS framework coding standard

- Integrate Often: Compile the code every day and test it in the system to ensure that the codes are compatible with the Aeronautical solution.

\section{- Testing}

- Unit Test: Create unit testing for the Geoprocessing tools

\subsection{Audience}

Target audience includes people with aeronautical knowledge such as aeronautical charting team members, aeronautical chart analysts, aeronautical project managers, aeronautical project developers, pilots and terminal control tower crew members. A 
further, through perhaps secondary target audience includes people who are interested in geoprocessing tool development and software development cycle such as the software developers, software project managers and software testing team members. 


\subsection{Overview of the Rest of this Report}

The rest of the project is divided into six chapters. Chapter two includes the project background and a literature overview. Chapter three describes the system analysis and design which involves the system scope, the system requirement and the project plan. Chapter four designates the database design. Chapter five goes over the Geoprocessing tool background and design. Chapter six details the implementation, which includes the program structure, the function calls and error handling. Chapter six covers the testing, the result and the documentation. The last chapter is on the project conclusion and future work. 



\section{Chapter 2 - Background and Literature Review}

This chapter starts with a discussion of the transformation of information from pilot personal notes to paper aeronautical charts and then on into digital format. During this discussion, some well known aviation agencies and associations will be depicted, as well as their establishment and why they mandated this transformation process. The next section introduces the OIS specifications from the aviation agencies. The following section introduces GIS technology, including its background and how this technology can help to solve the problem this project addresses. With the discussion of the OIS and GIS, the next section goes into a detailed investigation of past research and how that research connects to the Obstruction Identification Surface Aerodrome project. The final section summarizes this literature review.

\subsection{Paper to Digital}

In the early $20^{\text {th }}$ century, there were no formal aeronautical charts even though the skies were already filled with aircraft. Early pilots used their personal notes to help them navigate to and land at increasingly far away airports. They exchanged their personal notes, which made the aeronautical information become more solid and complete. However, even with the best information and notes, air travel was still restricted by visibility. A tremendous change occurred in 1941, when the first instrument approach and landing charts were developed. These enabled pilots to travel and land in low visibility situations. The demand for aeronautical charts soon increased dramatically, and after a couple of decades, it became obvious that standardized products and symbology were needed to sustain international air travel (McGaugh, 2004, p. 3). 
Since then, many aeronautical and aviation agencies and associations have been established. One of the global voices is the Federal Aviation Administration (FAA) in the United States. The mission of the FAA is to provide the safest, most efficient aerospace system in the world. Another leading aviation agency, the International Civil Aviation Organization (ICAO), was established in 1944 by 52 nations whose aim was to ensure a safe, orderly, and economic development of international air transport. Both agencies continuously strove to improve air safety. The FAA published Federal Aviation Regulations; the ICAO published Annexes. These regulations and annexes govern all aviation activities in order to maintain air safety (McGaugh, 2004, p. 4).

Besides the management of aviation activities, ensuring all aircraft are flying on the same data is also very important to air safety. The procedure of producing global aeronautical charts required a large number of aeronautical analysts and quality assurance specialists. Furthermore, chart maintenance requires a momentous effort and tremendous knowledge. There are also many modifications, including monthly annotations and graphics in global aeronautical charts. With so many changes and the possibility of human error at many stages of production, a GIS solution is the obvious choice in helping to solve this critical problem. This project, Aerodrome Obstruction Identification Surface, is a significant step forward in creating the GIS solution (McGaugh, 2004, p. 5).

\subsection{Obstruction Identification Surfaces}

OIS are imaginary surfaces with three-dimensional geometry. They are established in relation to the airport and to each runway. OIS provides two main functions: to identify which objects are obstructions around the airport, based upon whether or not said objects penetrate the OIS; and to control airplane flight altitude. If pilots know the location of 
obstructions around the airfield, they will have more precise data on how to control the flight altitude, hence avoiding an accident or near miss. There are two specifications involved in this project, and both specifications contain several similarities and differences. The similarities are that the measurement for both specifications is horizontal, starting from the runway centerline and the trends of both specifications extend outward and upward. The differences include the different units of measurement in both specifications, the angular units are unlike each other, and some terms used in both specifications are dissimilar.

\subsubsection{Obstruction Identification Surfaces Specification from the FAA}

The latest edition of FAR part 77 published by the FAA in March, 1993, describes the objections affecting air navigation. The purposes of FAR part 77 are to consolidate the obstruction standards for use by the FAA, to make them more efficient for determining the potential hazardous effect of future construction on air navigation, to evaluate the consequence of construction on operating processes, and to realize mitigating measures to improve safe air navigation. FAR part 77 is compiled in six subparts: general, notice of construction or alteration, obstruction standards, aeronautical studies of effect of proposed construction on navigable airspace, rules of practice for hearings under subpart $\mathrm{D}$, and establishment of antenna farm areas.

The subpart C, obstruction standards, is one of the two main sources for this project. It institutes standards for determining obstructions to air navigation and establishes the airport imaginary surfaces with relation to the airport and to a specific runway. The size of imaginary surfaces depends on two criteria: runway type and approach type. The runway type includes utility, visual, non-precision instrument, and 
precision instrument. The approach types are visual, non-precision instrument, and precision instrument. The five types of imagery surface set forth by FAR part 77, subpart C, are primary, horizontal, conical, approach, and transitional.

The primary surface is a rectangular shape around the airport runway centerline. The length of the primary surface is same as the length of the runway plus an additional 200 feet beyond each end of the runway if a clear way exists. The width of the primary surface for utility runways is 250 feet with only visual approaches and 500 feet with nonprecision instrument approaches. For visual runways with only visual approaches, the width of the primary surface is 500 feet. For non-precision instrument runways, the primary surface is 1,000 feet wide with non-precision instrument approach and visibility minimums as low as three-fourths of a statute mile. This width also applies to precision instrument runways. If the visibility is greater than three-fourths of a statute mile, the width for non-precision instrument runways is 500 feet (FAA, 1993, pp. 29, Section 77.25 (C) Primary Surface).

The approach surface is a surface extending outward and upward from each end of the primary surface. The width of the inner edge of the approach surface is the same width as the primary surface. The width of the outer edge of the approach surface is 1,250 feet for utility runways with only visual approaches, 1,500 feet for non-utility runway with only visual approaches, 3,500 feet for non-precision instrument runway with visibility minimums greater than three-fourths of a statue mile, 2,000 feet for utility runways with non-precision instrument approach, 4,000 feet for non-precision instrument runways with non-precision instrument approach and visibility minimums as low as three-fourths statute mile, and 16,000 feet for precision instrument runways. The length 
of the approach surface is 5,000 feet for all utility and visual runways, 10,000 feet for all non-precision instrument runways, and a first section of 10,000 feet plus a second section with an additional 40,000 feet in different slope value for all precision instrument runways. The slope of approach surface is 20 to 1 for all utility and visual runways, 34 to 1 for all non-precision instrument runways, 50 to 1 for the first section, and 40 to 1 for the second section for all precision instrument runways (FAA, 1993, pp. 29 - 30, Section 77.25 (D) Approach Surface).

The horizontal surface is relatively simple. It is a horizontal plane placed 150 feet above the runway centerline. The boundary is formed by swinging arcs in a given radius from the center point of each end of the primary surface and connecting the arcs by lines tangent to those arcs. The designated radius for all utility or visual runways is 5,000 feet, and 10,000 feet for all other runways. Conical surface and transitional surface are the simplest surfaces in FAA OIS. Conical surface is a surface that extends outward and upward from the external boundary of a horizontal surface. The slope conical surface is 20 to 1 for 4,000 feet. Transitional surfaces are the connection surfaces to the primary, horizontal, approach, and conical surfaces. These surfaces extend outward and upward at a slope of 7 to 1 (FAA, 1993, pp. 29, Section 77.25 (A) Horizontal Surface) (FAA, 1993, pp. 29, Section 77.25 (B) Conical Surface) (FAA, 1993, pp. 30, Section 77.25 (E) Transitional Surface).

\subsubsection{Obstruction Identification Surfaces Specification from ICAO}

Annex 15 was first published by the ICAO more than half a century ago. It defines how an aeronautical information service (AIS) shall receive, collate, edit, store, and distribute aeronautical information. Regularity and stability are the goals of Annex 15 in the 
provision of aeronautical information that is mandatory for operational use by international civil aviation. In recent years, the amendments to Annex 15 have mirrored the increased requirement for the prompt provision of quality aeronautical information and terrain and obstacle data as they have become significant components of on-board navigation systems. After much effort putting the available material together from the Radio Technical Committee for Aeronautics (RTCA) and European Organization for Civil Aviation Equipment (EUROCAE), a new chapter entitled "Electronic Terrain and Obstacle Data" (eTOD) was added to Annex 15. This new chapter contains provisions for the eTOD function, coverage, eTOD numerical requirements, content, and structure of eTOD databases, data product specifications for eTOD and availability. To conform to the distinguished user requirements for electronic terrain data while taking into account cost-efficiency, attainment methods and data accessibility, it is proposed that eTOD be provided according to four basic coverage areas. Proposed Area 1 covers the whole territory of a state. Proposed Area 2 has coverage over the established terminal control areas designated by one of two boundaries, either a terminal control area or an area covering a 45 kilometer radius around the airport, whichever is smaller. Area 3 extends from the edge of the runway to 90 meters from the runway centerline and for all other parts of aerodrome or heliport movement area, 50 meters from the edge of the defined area. Area 4 is for category two or three runways only. The area is a rectangular shape with a width of 60 meters on either side of the extended runway centerline and a length of 900 meters from the runway threshold measured along the extended runway centerline (International Civil Aviation Organization, 2004). 
Timing is very important to the eTOD project and is also the main challenge to all contracting states. Annex 15 section 10.6 states that by November 20, 2008, eTOD were to be made available in accordance with Area 1 specifications and terrain data in accordance with Area 4 specifications. In addition, by November 18, 2010, eTOD were to be made available in accordance with Area 2 and Area 3 specifications. Based on the cut-off date for all eTOD areas availability, Area 1 and Area 4 developments were more easily accomplished when compared to Area 2 and Area 3. Between the Area 2 and Area 3 specifications, Area 2 specification is more complicated because the shape of the coverage Area 2 is three dimensional. In spite of the complex geometry of Area 2, development of Area 2 surfaces is the main goal of this project and Area 2 specification is the main source of this project (EUROCONTROL, 2009).

\subsection{Geoprocessing}

Geoprocessing (GP) is a GIS operation used to manipulate GIS data. Some people restrict this definition to the operations in a GIS that perform spatial analysis. Other people argue that this definition is too narrow and all operations that accept input and generate output are GP operations. To determine which operations are considered GP operations, most people agree that a typical GP operation takes a set of inputs, performs an operation on the input, and returns new information generated by that operation. Despite the ambiguity of GP definitions, GP is what people use for many day-to-day tasks. The reason for this is that there are many advantages to GP (ESRI).

User interface consistency is one of the major advantages in GP. A user who has performed a "Clip", "Project" or "Buffer" GP function is already familiar with the interface of every other GP tool. User interface flexibility is another major advantage. 
Any GP function that is written properly gets a dialog and a command-line string automatically, and can be run from outside ArcGIS in a standalone Python script. The user can schedule a huge and complicated GP function and let it run overnight. Furthermore, every tool can easily be run in batch and with no extra work for the developer. User interface validation is vital in terms of development and usage because any properly written GP function will have nearly airtight protection from invalid input. In other words, a user cannot crash the program. Parameter validation is simple and much of it is already done for the developer by many types of input domains and data types available in GP. In addition, there is an existing error framework, with meaningful severity levels, for getting output to the user in the form of GP messages. It is simple for a user to see why something failed, or to warn the user that something unpredictable has happened (ESRI, 2006).

\subsection{Past Research}

Obstruction identification surface has been defined for more than 40 years. Many organizations use it for planning, identifying obstructions, developing runway approach procedures, charting, and surveying.

The National Oceanic and Atmospheric Administration (NOAA) published a study called FAA Airport Surveying - GIS Program. It is about airport surveying and introduces a geospatial digital version of Airport Obstruction Charts (AOC). An AOC, a 1:12,000 scale chart, is a representation of objects that penetrate the OIS described in FAR part 77. It also displays airplane movement, navigational aids, buildings, and roads around an airport, and other planimetric details in an airport's surrounding area. 
In the past, the AOC was produced in a non-georeferenced format which imported the data sources from the NOAA Obstruction Chart Database, and exported to the Computer-Aided Design (CAD). The CAD file was then plotted after cartographically manipulating it onto a template. The new digital AOC is a compilation of geospatial data, including the data relating to imaginary surfaces that define safe altitudes for approaches and landings. NOAA developed a data content standard for all these features. It includes geometry type, a description, attributes, and an abbreviation. Although OIS data such as the geometry type, generation process and collection method were not presented in detail in this study, it is still an excellent example of aeronautical charting transformation from paper to digital with the use of OIS (Vidal, Susan; Graham, Douglas; Steele, Jeffrey;).

While the above study did not thoroughly explain OIS generation, another study published by Ray L. Harris Jr. and Andrea R Johnson presented a new approach to detect airfield vertical obstructions using digital photogrammetry and GIS. (Harris \& Johnson) This study also introduced commercial off-the-shelf software called ClearFlite which generates the OIS model in less than five minutes. While ClearFlite is technically highly developed, it has several drawbacks. The most important drawback is ClearFlite has limited capabilities. The OIS GP tool can chain up other GP tools to perform more complex operations and calculations. This tool also has the ability to run in server environment and becomes a useful internet application. Mark Belrose, in his study, Identification and Characterization of Obstruction to Flight in March Air Reserve Base, also points out two more drawbacks to ClearFlite. The first drawback he mentions is that the software is relatively expensive. The second drawback is that ClearFlite is not 
standalone software, but an extension and must be used in grouping with BAE's SOCET SET software (Belrose, 2005).

\subsection{Summary}

This chapter started with a summary of the transformation of aeronautical data from paper to digital. Next, two OIS specifications published from FAA and ICAO were delineated in section 2.2. Both specifications have similarities and differences. These similarities and differences are the major factors determining the database design, the GP design, and the implementation. The concept and background of the GP outlined in section 2.3 greatly benefited this project by including the key components of the GP and the relationship between them. The previous section discussed the OIS usage and research from past studies. The next chapter sketches out the system design and project plan to meet the requirements of this project. 


\section{Chapter 3 - Systems Analysis and Design}

The previous chapters presented the introduction to this project, the background for the OIS, and the concept of GP. In this chapter, a brief statement of the essential problem to be solved by the OIS project is laid out. It then goes on to discuss the functional and nonfunctional requirements of the project, as well as the system architecture and the system design. Finally, this chapter introduces the project plan and addresses the major differences between the planned and actual time to complete the major task.

\subsection{Problem Statement}

A safe air travel environment is very important to both the economy and to national security. The International Civil Aviation Organization has dictated that member states produce eTOD sets by 2010. ESRI's Aeronautical Solution recognizes that their customers need a system to assist them in collecting, manipulating, and analyzing the data. More importantly, data sets must be generated for each airport corresponding to the specifications of four specific geographic coverage areas. After months of effort, an eTOD data set was clearly defined for each airport corresponding to the outlines of four specific geographic coverage areas delineated by ICAO rules.

With the eTOD data set, the ESRI Aeronautical Solution team now faces the challenge of finding a method to generate the OIS effectively in order to have a clear representation of vertical obstruction. An object is considered a vertical obstruction if it penetrates the OIS. With these obstacle data, pilots can make the best decision as to how to approach or depart from an airfield. In addition, airplane technicians and control tower operators can determine the maximum allowable takeoff weight for a particular runway. 
Traditionally, the creation and maintenance of OIS features have been performed using computer-aided drafting (CAD) systems. This is a time consuming method and it may contain human errors. The ESRI Aeronautical Solution team proposed to develop a GP tool to simplify and improve the OIS generating process. In this instance, to simplify means having fewer buttons click and to improve means to decrease the processing time. The OIS GP tool, combined with the OIS specification from FAA and eTOD, was specifically designed for more efficient creation and management of OIS features.

\subsection{Requirements Analysis}

The goals of the OIS project were ease of use, a user friendly interface, and generation of the OIS effectively while minimizing possible human error. There were several requirements to accomplish the above purposes which can be classified into two categories: functional and non-functional requirements.

\subsubsection{Functional Requirements}

The major components for this project are a GP tool, a testing solution, and a final testing report. During the discussion with the client it was decided that a GP tool would be a good solution for the OIS polygon feature generation because it is easy to use, has a consistent user interface, can be executed from server and command prompt, and has the ability to chain up with other GP tools in geoprocessing model builder to perform more complex tasks.

All the generated OIS features will be stored in a file geodatabase and used for further development by the ESRI Aeronautical Solution team. In addition, two TIN surfaces will also be derived from both FAA and eTOD specification. A testing solution and a final testing report allow for a smooth transition and easy maintenance. The testing 
solution is embedded in the unit testing framework. In addition, the testing procedures and the final testing report follow the ESRI's Aeronautical Solution testing team's current testing procedure and testing report format. The following table lists the functional requirements and their descriptions.

Table 1. Functional Requirements

\begin{tabular}{|l|l|}
\hline \multicolumn{1}{|c|}{ Function } & \multicolumn{1}{c|}{ Description } \\
\hline OIS GP tool & $\begin{array}{l}\text { The GP tool generates the OIS polygon features based upon } \\
\text { user input }\end{array}$ \\
\hline OIS features & A file geodatabase stores the generated OIS features \\
\hline OIS TIN surface & Two TIN formatted surfaces derived from the OIS features \\
\hline Unit testing solution & The system tests the functions and procedures in the GP tool \\
\hline
\end{tabular}

\subsubsection{Non Functional Requirements}

Non-functional requirements are sub-classified into three categories: technical requirements, operational requirements, and transitional requirements.

The technical requirements for this project included appropriate software and extensions. The GP tool was developed under the PLTS and Aeronautical Solution environment. PLTS for the ArcGIS solution includes Foundation, which provides the database editing, quality control, and automated data migration capabilities for each solution. Many base classes are built and developed in PLTS Foundation. The source code of Aeronautical Solution inherited from PLTS source code provides customized tools and functionalities to enable database-driven cartographic production of aeronautical charts. Because of privacy and security issues, the OIS project was 
developed at the client site as the client could provide the best resources for the project. As per ESRI's request, the GP tool ArcInfo version 9.3.1 was used with the Aeronautical solution extension as the backbone of the solution, and was compatible with version 10 .

For the operational requirement, it was assumed that the users would have adequate GIS experience, as well as the aeronautical knowledge for using this GP tool. As such, the tool design and the workflow for using this tool were carefully considered, as the OIS GP tool would not benefit the user if poorly designed. Another operational requirement is the International Organization for Standardization (ISO). ISO9001:2000 specifies requirements for a quality management system where an organization needs to demonstrate its ability to consistently provide products that meet customer and applicable regulatory requirements, and aims to enhance customer satisfaction through the effective application of the system, including processes for continual improvement of the system and the assurance of conformity to customer and applicable regulatory requirements. All the design, implementation, and testing for this project needed to meet the ISO standard as the Aeronautical solution at ESRI is ISO 9001:2000 certified.

Transitional requirements focused on the needs of the client when shifting into using the GP tool. Major requirements were the testing solution, exception handling, and documentation. The testing solution implemented included the inline documentation and the final testing report.

The above nonfunctional requirements are summarized in the following table with the function names and descriptions. 
Table 2. Non-Functional Requirements

\begin{tabular}{|c|c|}
\hline Function & Description \\
\hline Technical & \\
\hline Computing environment & $\begin{array}{l}\text { Desktop or laptop computer. Aeronautical and PLTS } \\
\text { development network system }\end{array}$ \\
\hline Software & $\begin{array}{l}\text { ArcGIS 9.3.1 with Aeronautical Solution extension and } \\
\text { compatible to version } 10\end{array}$ \\
\hline User interface & ArcToolbox \\
\hline Operational & \\
\hline User expertise & Adequate GIS experience and Aeronautical knowledge \\
\hline Development standard & PLTS standard \\
\hline Transitional & \\
\hline Documentation & Inline documentation and tool design documents \\
\hline Exception & Handling the unexpected error \\
\hline Testing the GP tool & Test the functionality of the GP tool \\
\hline
\end{tabular}




\subsection{System Design}

This section focuses on the computer software and hardware configurations selected for the project. The following table lists the components needed for developing the OIS GP tool. All the components were high priority and at the same level.

Table 3. Project Component

\begin{tabular}{|l|l|}
\hline \multicolumn{1}{|c|}{ Component } & \multicolumn{1}{c|}{ Description } \\
\hline OIS Specification & $\begin{array}{l}\text { The specification described the area and slope for different } \\
\text { type of OIS polygon features }\end{array}$ \\
\hline $\begin{array}{l}\text { Production Line Tool Set } \\
\text { (PLTS) Aeronautical } \\
\begin{array}{l}\text { Solution development } \\
\text { guideline }\end{array}\end{array}$ & $\begin{array}{l}\text { The guideline is used for keeping all the developing } \\
\text { procedures to meet the standard }\end{array}$ \\
\hline $\begin{array}{l}\text { ESRI ArcObject class } \\
\text { library }\end{array}$ & $\begin{array}{l}\text { The programming library that was used for developing the } \\
\text { GP tool }\end{array}$ \\
\hline
\end{tabular}

ESRI provided a high end laptop with an Intel Core 2 CPU, 2 GB Ram, NVIDIA Quadra FX2500M graphics card, and 75 GB HHD for the GP tool implementation. Another desktop was also provided for testing and backup purposes with the same configuration and a larger HHD. The above system contained adequate configurations for the following software: ArcGIS 9.3.1, PLTS 9.3.1, Aeronautical Solution 9.3.1, ArcGIS SDK (.NET Framework, C++, VB6), MS Visual Studio 2005, and NUnit. Due to security issues, ESRI set up network access for the implementation of the OIS GP tool.

There was no particular study area as the focus of this project; hence the Geodatabase design mainly focused on the output OIS polygon features. The details are described in Chapter 4. The following diagram describes the workflow of the OIS generation. The detailed workflow and procedures are described in Chapter 5. 

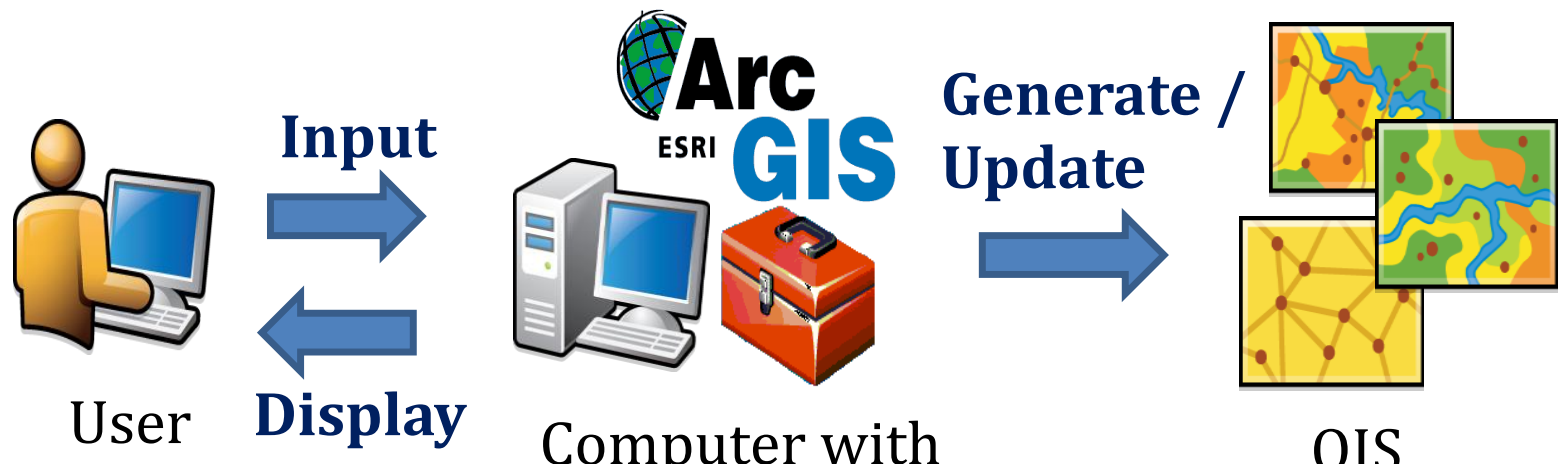

User

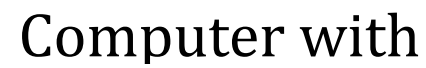

OIS

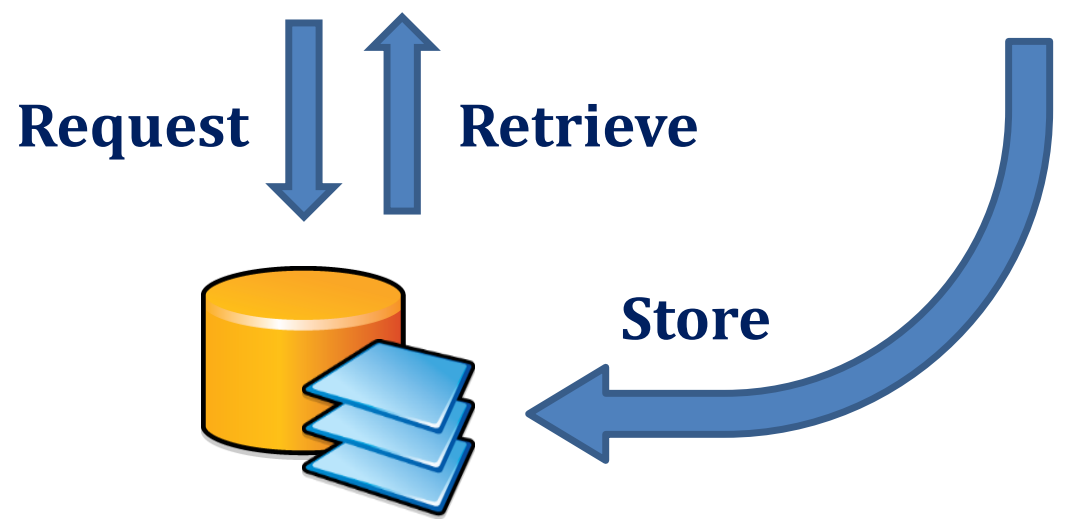

File

Figure 3: System Functionalities Diagram 


\subsection{Project Plan}

This section presents the original plan of this project. The main and subtasks found in the original plan will also be described in detail, and the timeframe associated to the tasks is presented in the following Gantt chart.

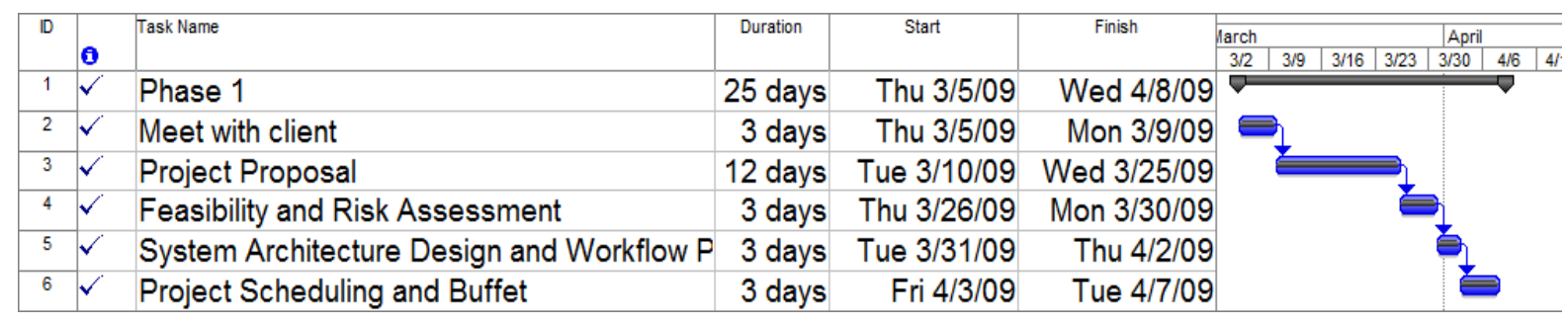

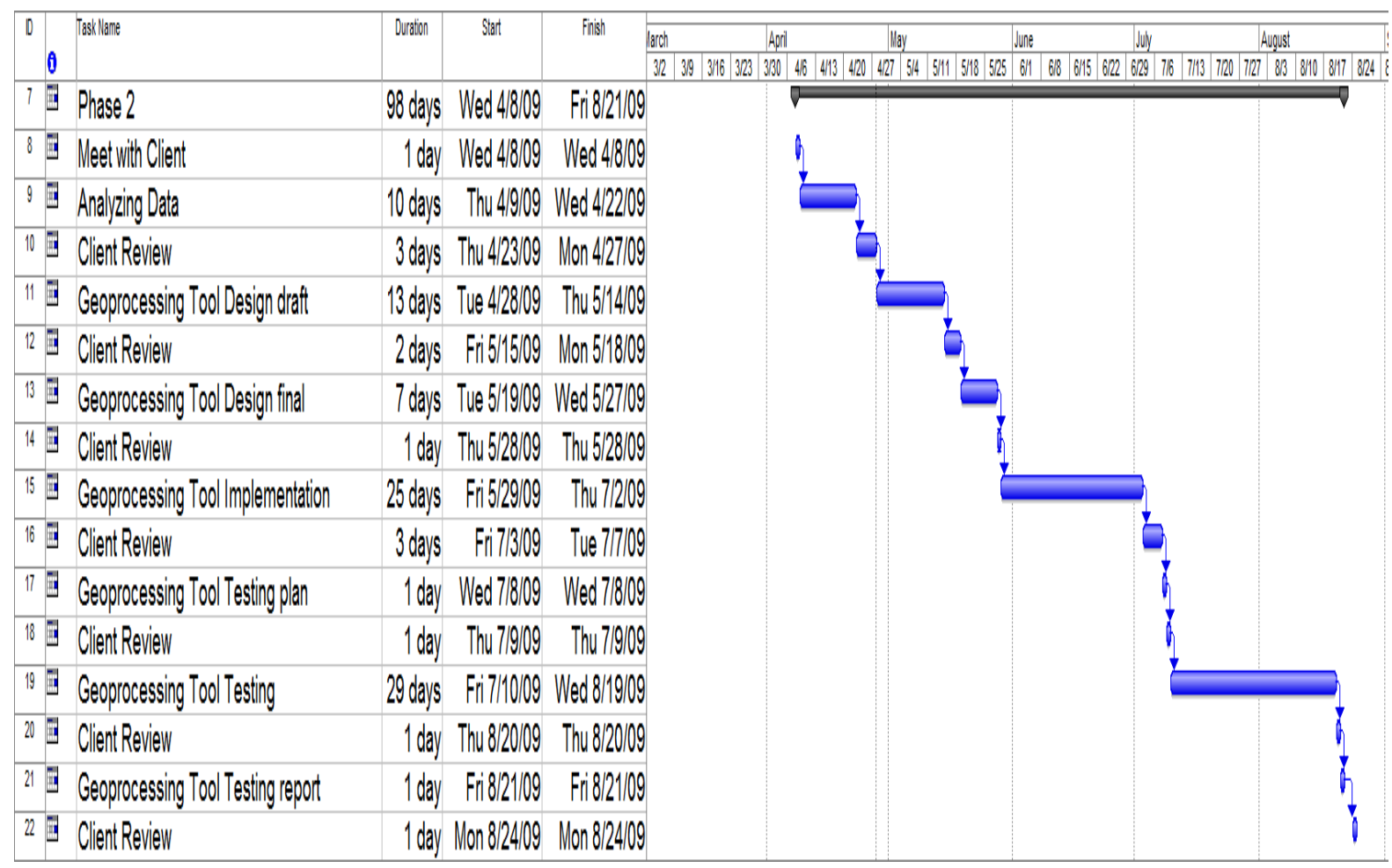

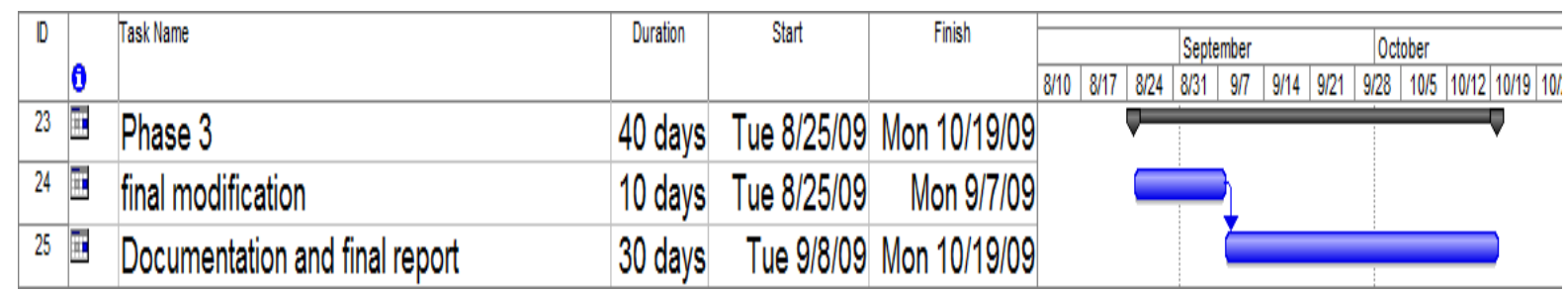

\section{Figure 4: Gantt chart for OIS Project}


The workflow diagram in figure 5 lists out the major tasks required for developing and completing the project.

- Meet with client - Schedule weekly meetings with client to make sure both sides are on the same page.

- Data analysis - Consult with the product engineer and database administrator of the aeronautical team.

- Design the GP tool - Read the geoprocessing tool development documents and fully utilize the resources from the ESRI development web page.

- Implementing the GP tool - Follow the Production Line Tool Set coding standard and submit request for code review from the developers on the aeronautical team.

- Testing the GP tool - Set up the testing plan and build the unit testing for the GP tool. Consult the testing developer in the aeronautical team for more detailed information.

- Documentation - Write a design plan for the GP tool and document all the modules. The documentation will follow the Production Line Tool Set documentation standard.

- Deliverable - The delivery method for the OIS GP tool design documents and the final report will be sent through email. These documents will also be provided in hardcopy if requested. The OIS GP tool will be developed in the aeronautical solution structure and stored on compact disk when it is completed. 


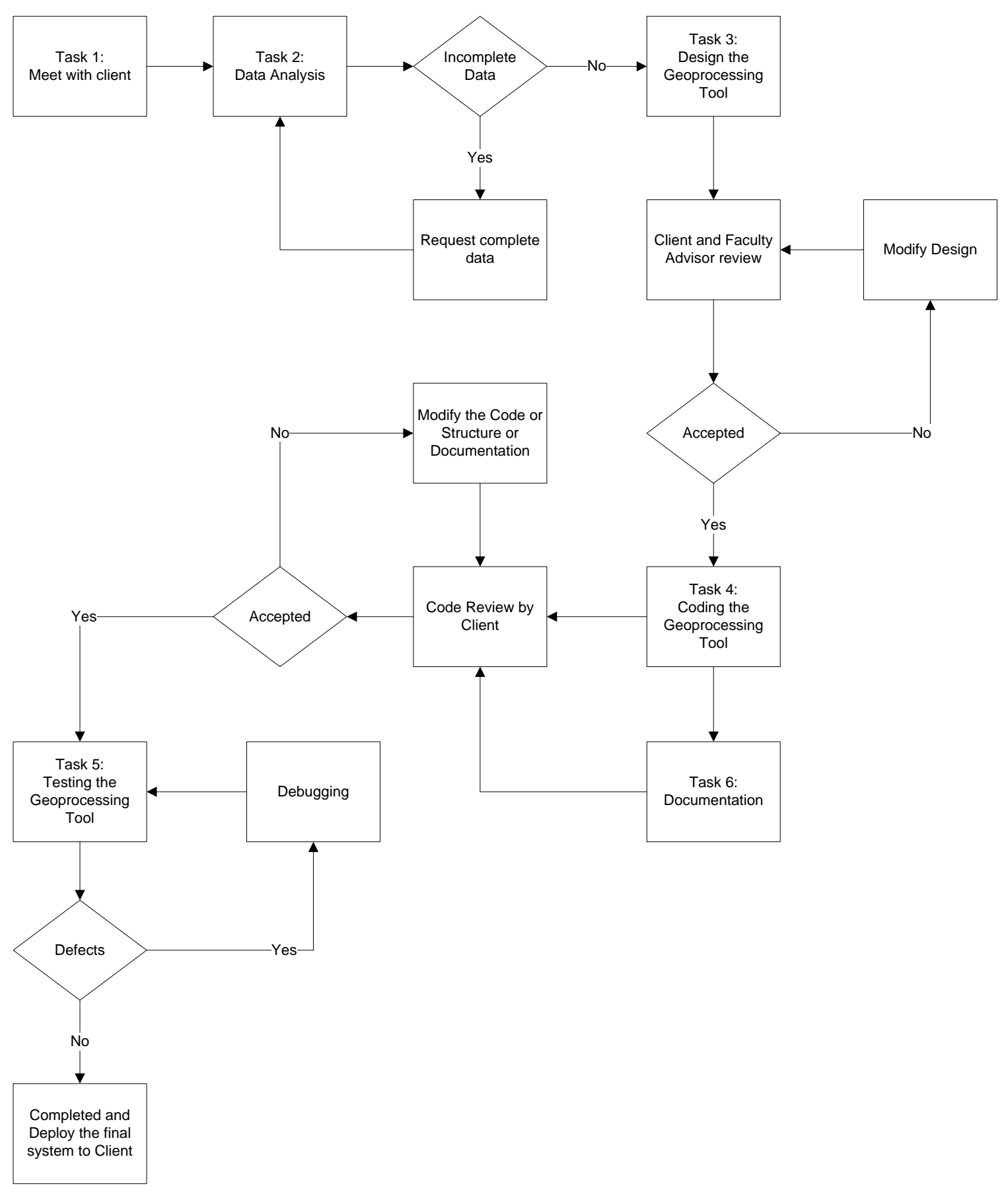

\section{Figure 5: Workflow Diagram}

This project mostly followed the Extreme Programming (XP) methodology because Extreme Programming Practices lead to a development process that is more responsive to customer needs than traditional methods, creating software of better 
quality. The following table depicts the rules and practices of Extreme Programming, these rules and practices can be applied and be executed well in this project:

Table 4. Extreme Programming rules and practice

\begin{tabular}{|c|c|}
\hline Rules and Practices & Description \\
\hline \multicolumn{2}{|l|}{ Planning } \\
\hline User stories & Meet with ESRI employee in Redlands \\
\hline $\begin{array}{l}\text { Make frequent small } \\
\text { releases }\end{array}$ & $\begin{array}{l}\text { Deploy the prototype OIS GP tool to Redlands in order to get } \\
\text { valuable feedback in time to have an impact on the system's } \\
\text { development, while the OIS specification is still in drafting } \\
\text { status }\end{array}$ \\
\hline Fix XP when it breaks & $\begin{array}{l}\text { Due to the complexity of the project and the dependency } \\
\text { upon the ESRI Production Line Tool Set (PLTS) framework, } \\
\text { the project may require adjustment and modification during } \\
\text { the production cycle period }\end{array}$ \\
\hline \multicolumn{2}{|l|}{ Designing } \\
\hline Simplicity & Keep the GP tool as simple as possible \\
\hline $\begin{array}{l}\text { Choose a system } \\
\text { metaphor }\end{array}$ & $\begin{array}{l}\text { Inherit from the ESRI PLTS framework and ESRI } \\
\text { Aeronautical Solution }\end{array}$ \\
\hline $\begin{array}{l}\text { No Functionality is added } \\
\text { early }\end{array}$ & $\begin{array}{l}\text { Concentrate on currently scheduled requirements instead of } \\
\text { potential future needs and/or extra flexibility }\end{array}$ \\
\hline \multicolumn{2}{|l|}{ Coding } \\
\hline $\begin{array}{l}\text { Code must be written to } \\
\text { agreed standard }\end{array}$ & Code will follow the ESRI PLTS framework coding standard \\
\hline Integrate often & $\begin{array}{l}\text { Compile the code every day and test it in the system to } \\
\text { ensure that the codes are compatible with the Aeronautical } \\
\text { solution }\end{array}$ \\
\hline \multicolumn{2}{|l|}{ Testing } \\
\hline Unit Test & Create unit testing for the GP tools \\
\hline
\end{tabular}




\subsection{Summary}

The successful project execution was a direct result of the system analysis and design that were set up during early planning stages. The previous sections outlined the necessary components for the system analysis and design. A requirement analysis was established by developing a detailed understanding of the goals and objectives of this project. A complete system design was created to illustrate the project architecture and workflow. The project plan outlined the steps for effectively implementing the functional requirements and successfully completing the project. This chapter set out general expectations for the project. In the remaining chapters, the discussion will detail the database design and the GP tool design, followed by the implementation and result analysis. 


\section{Chapter 4 - Database Design}

At the start of this project, the database design seemed to be a low priority because a completed GP tool that can easily generate the OIS features was the main goal. Also the existing aeronautical data model was already well developed and complicated, so it was not easy to modify all the classes, tables, and the relationships between them. On the other hand, a well designed and organized database was the key to executing the project successfully. Further, the input data type for the GP tool in this project had to be explicitly defined in the database. And finally, maintaining the OIS features in a global and central format was one of the objectives for this project. With that determined, the database design is still a crucial component in this project.

This chapter introduces the sequential steps used to determine what data was required, how it was organized, where it was obtained, and how it was prepared for use. All levels of database modeling are also explained. Modeling was undertaken on the conceptual, logical, and physical levels. Each level of design is documented in the sections below. In addition to the input data, this chapter also describes the output data, including fields, format, and type.

\subsection{Conceptual Data Model}

The conceptual data model defines entity names that are significant to a project, as well as the relationship between the entities. As previously stated, the aeronautical data model already exists and is complicated. Consequently, this project inherited from another aeronautical data model which is simplified from the existing one. The simplified version

of aeronautical data model was developed by David Dale Robinson. This model was used 
in an eTOD database to facilitate the easy transfer of terrain and obstacle data to a final GIS eTOD solution. It contained minimal data sets and was relatively simple. The integration of the data model from this project to the simplified aeronautical data model is discussed in the physical data model section.

The first step in designing a database was to examine the functional requirements for the GP tool, as well as determining what entity was needed for the data model and how to integrate the entity into the simplified aeronautical data model. The functional requirements for the GP tool were twofold. First, a user needs to input the airport name and the runway number, and then specify the type and specification. Second, the GP tool generates the OIS features based on user input and stores the features in a geodatabase. To accomplish the first requirement, the significant entities to input in this project were airports, runways, and OIS specifications. To achieve the second requirement, there are two entities to separately store: the FAA OIS and eTOD OIS.

In the conceptual data model, another essential process was to find out the relationship between entities. The reason for that is because an entity needs evidence to prove its existence in a database. Since there are only five entities in this project, the relationship between the entities can easily be discovered. First, there is a direct relationship between the airport entity and the runway entities. Second, OIS is generated based on the airport runway; therefore, the OIS entity for both FAA and eTOD is related to the runway. Finally, the OIS specification entity holds one type of OIS and hence, they are related to each other. Figure 6 shows the conceptual data model based on the above entities and relationships. 


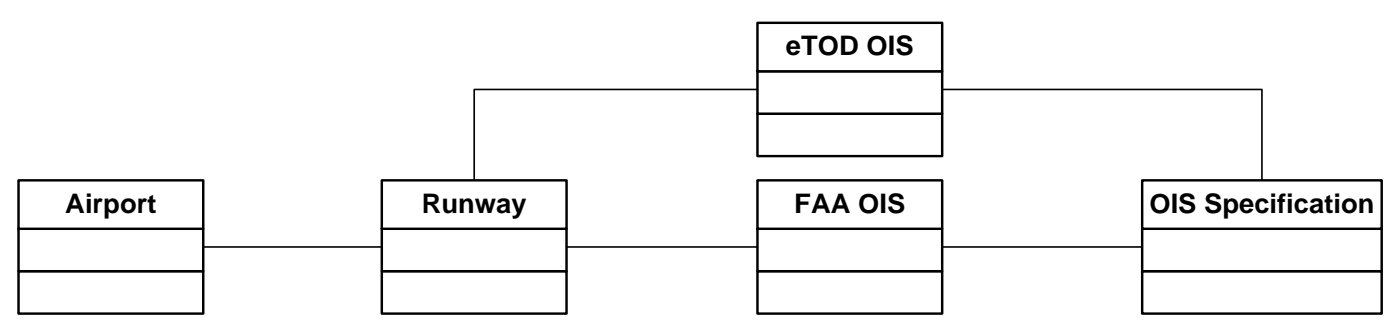

\section{Figure 6: Conceptual Data Model}

After the analysis of the function requirements for the GP tool was completed, there were five entities needed for the database design. When comparing these five entities to the simplified aeronautical data model, there were three additional entities that were needed to form the OIS data model. These three entities are the OIS specification, the FAA OIS, and the eTOD OIS.

The entities and their relationship to one another defined the OIS data model on a more general level. As such, they are considered to be and are used as the conceptual data model. After discussion with the client, the entities and their relationships were finalized and accepted. The logical data model began here, guided by this conceptual data model design.

\subsection{Logical Data Model}

The logical data model was developed from the hypothetical framework of the conceptual data model. It increases the complexity by depicting the data attributes and by describing those entities and the relationships between them.

The airport entity has three attributes that are important to the OIS features: the airport name, the state name, and the city name. The runway entity contains two attributes: the runway number and its length. The attributes of OIS specification entity are 
the type and the measurement of the OIS. FAA OIS and eTOD OIS entities also include the airport name, the runway number, and the type of OIS.

Once the entities and attributes are established, the relationship between the entities can be interpreted. The first consideration is the relationship between the airport entity and the runway entity. An airport may contain one or more runways and therefore, the relationship between the airport entity and the runway entity is a one-to-many relationship. For the runway entity and both OIS entities, each runway has one FAA OIS or one eTOD OIS, depending on the government aviation regulation. Thus, the relationship between them is a one-to-one relationship. A one-to-one relationship also exists between the OIS specification entity and both the OIS entities, as each OIS is guided by one type of OIS specification. Figure 7 illustrates the logical data model for this project.

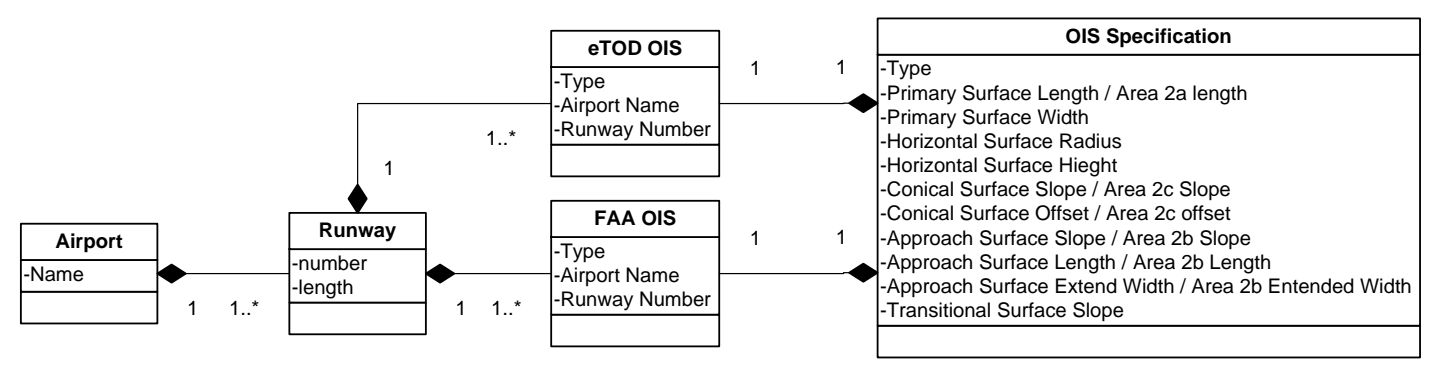

\section{Figure 7: $\quad$ Logical Data Model}

Once the logical data model is in place, a completed database design is one step closer. Processes not yet in the logical data model design are the formalization of table and attribute names, the design of attribute data types, and the initialization method for the new records. These processes are the key to the formation of the physical data model. This is discussed in the next section, as it pertains to finalizing the database design. 


\subsection{Physical Data Model}

Once a logical data model had been completed, physical data modeling was the last step in carrying the database to completion. To begin the physical data model design, table name, attribute name, attribute data type, and the method of initializing are needed.

The table name for an airport entity is called ADHP. ADHP officially stands for Aerodrome or Heliport. It is a point feature class that represents the geographic location of an airport. More specifically, it is the geographic center point of the airport covered by the active runways. The attribute, Name_txt, in ADHP feature class is used to identify the location of the airport. This attribute is vital to this project as it will be populated in the OIS feature table to identify which OIS feature belongs to which ADHP. The data type of Name_txt is string.

Table 5. Airport Feature Class Summary

\begin{tabular}{|c|l|l|l|l|}
\hline Entity & $\begin{array}{c}\text { Table } \\
\text { Name }\end{array}$ & \multicolumn{1}{|c|}{$\begin{array}{c}\text { Significant } \\
\text { Attribute }\end{array}$} & $\begin{array}{c}\text { Attribute Data } \\
\text { Type }\end{array}$ & Attribute Description \\
\hline Airport & ADHP & Name_txt & String & $\begin{array}{l}\text { Indicate the name of the } \\
\text { airport }\end{array}$ \\
\hline
\end{tabular}

A runway entity is fundamental to the OIS generation. Normally the centerline of a runway represents the runway, beginning on one side of a runway threshold and extending to the opposite runway threshold. This centerline is represented by the ADHPSurfaceline in aeronautical data. The runway centerline is one of three surface line subtypes: runway, final approach and takeoff area (FATO), and taxiways. The significant attribute in ADHPSurfaceline for this project is the global format identifier (GFID) which is used to categorize the OIS to the particular runway and is a string with a combination of characters and numbers. When considering the significant attributes in 
ADHPSurfaceline, the elevation is a major factor in this project because it affects all the calculation and the height of surfaces. Furthermore, this attribute is a major issue in ADHPSurfaceline feature class in aeronautical data, at least in the client's aeronautical data. Currently the aeronautical data do not contain the elevation value or the $\mathrm{z}$-value. Therefore, the addition of the elevation attribute or redesigning the feature class is needed for ADHPSurfaceline. This discussion is in Chapter 6.

Table 6. Runway Feature Class Summary

\begin{tabular}{|l|l|l|l|l|}
\hline Entity & Table Name & $\begin{array}{l}\text { Significant } \\
\text { Attribute }\end{array}$ & $\begin{array}{l}\text { Attribute } \\
\text { Data Type }\end{array}$ & Attribute Description \\
\hline Runway & ADHPSurfaceline & GFID & String & $\begin{array}{l}\text { Runway identifier of the } \\
\text { airport }\end{array}$ \\
\hline
\end{tabular}

The OIS specification entity stores the type of OIS specification, either FAA or eTOD. OIS_Spec is the table name for the OIS specification and it contains several attributes: Type, Primary Surface, Horizontal Surface, Conical Surface, Transitional Surface, Approach Surface, Area 2a, Area 2b, and Area 2c. When comparing the surface description from both FAA and ICAO OIS specifications, there are several zones similar to each other. First is the primary surface and area 2a. Second is the approach surface and area $2 b$. Third is the conical surface and area $2 c$. Based on these similarities, those attributes can be combined into one attribute to represent both specifications. The Type attribute is one of two specification subtypes: FAA and eTOD. The data type of the Type attribute is string and other attributes are double. The following table summarizes the OIS specification table. 
Table 7. OIS Specification Table Summary

\begin{tabular}{|c|c|c|c|c|}
\hline Entity & $\begin{array}{l}\text { Table } \\
\text { Name }\end{array}$ & Significant Attribute & $\begin{array}{l}\text { Attribute } \\
\text { Data } \\
\text { Type } \\
\end{array}$ & $\begin{array}{l}\text { Attribute } \\
\text { Description }\end{array}$ \\
\hline \multirow[t]{11}{*}{$\begin{array}{l}\text { OIS } \\
\text { Specification }\end{array}$} & \multirow[t]{11}{*}{ OIS_Spec } & Type & Text & $\begin{array}{l}\text { Indicate the type of } \\
\text { OIS }\end{array}$ \\
\hline & & PS_Length & Double & $\begin{array}{l}\text { The length of } \\
\text { primary surface }\end{array}$ \\
\hline & & PS_2a_Wdith & Double & $\begin{array}{l}\text { The width of } \\
\text { primary surface and } \\
\text { area } 2 \mathrm{a}\end{array}$ \\
\hline & & HS_Radius & Double & $\begin{array}{l}\text { The radius of } \\
\text { horizontal surface }\end{array}$ \\
\hline & & HS_Height & Double & $\begin{array}{l}\text { The height of } \\
\text { horizontal surface }\end{array}$ \\
\hline & & CS_2c_Slope & Double & $\begin{array}{l}\text { The slope of conical } \\
\text { surface and area } 2 \mathrm{c}\end{array}$ \\
\hline & & CS_2c_Offset & Double & $\begin{array}{l}\text { The offset of conical } \\
\text { surface and area } 2 c\end{array}$ \\
\hline & & AP_2b_Length & Double & $\begin{array}{l}\text { The length of } \\
\text { approach surface and } \\
\text { area } 2 b\end{array}$ \\
\hline & & AP_2b_Slope & Double & $\begin{array}{l}\text { The slope of } \\
\text { approach surface and } \\
\text { area } 2 b\end{array}$ \\
\hline & & AP_2b_ExtendedWidth & Double & $\begin{array}{l}\text { The extended width } \\
\text { at the end of } \\
\text { approach surface and } \\
\text { area } 2 b\end{array}$ \\
\hline & & TS_Slope & Double & $\begin{array}{l}\text { The slope of } \\
\text { transitional surface }\end{array}$ \\
\hline
\end{tabular}

The FAA OIS and eTOD OIS store the output of the OIS and the table names for both the FAA OIS and eTOD OIS are FAA_OIS and eTOD_OIS respectively. These two tables also contain the same attributes: ADHP, ADHPSurfaceline, and Surface_Type. The Name_txt attribute in the ADHP feature class indicates the name of the airport. The 
GFID attribute in the ADHPSurfaceline feature class indicates that the OIS belongs to a particular runway. The Surface_Type attribute is one of five OIS subtypes for the FAA: primary, horizontal, conical, approach, and transitional. For eTOD, it is one of three OIS subtypes: $2 \mathrm{a}, 2 \mathrm{~b}$, and $2 \mathrm{c}$.

Table 8. FAA OIS and eTOD OIS Tables Summary

\begin{tabular}{|l|l|l|l|l|}
\hline Entity & Table Name & \multicolumn{1}{|c|}{$\begin{array}{c}\text { Significant } \\
\text { Attribute }\end{array}$} & $\begin{array}{c}\text { Attribute } \\
\text { Data Type }\end{array}$ & \multicolumn{1}{|c|}{ Attribute Description } \\
\hline \multirow{2}{*}{$\begin{array}{l}\text { FAA } \\
\text { OIS }\end{array}$} & \multirow{2}{*}{ FAA_OIS } & ADHP & String & $\begin{array}{l}\text { Indicate the name of the } \\
\text { airport }\end{array}$ \\
\cline { 3 - 5 } & ADHPSurfaceline & String & $\begin{array}{l}\text { The GFID of the airport } \\
\text { runway }\end{array}$ \\
\cline { 3 - 5 } & & Surface_Type & String & Specify the surface of OIS \\
\hline OIS & \multirow{2}{*}{ eTOD_OIS } & ADHP & String & $\begin{array}{l}\text { Indicate the name of the } \\
\text { airport }\end{array}$ \\
\cline { 3 - 5 } & & ADHPSurfaceline & String & $\begin{array}{l}\text { The GFID of the airport } \\
\text { runway }\end{array}$ \\
\cline { 3 - 5 } & & Surface_Type & String & Specify the surface of OIS \\
\hline
\end{tabular}

In this project, the integration of the designed database model to the simplified aeronautical database model is an additional process in the physical data model design. To begin the integration process, it is important to understand the structure of the simplified aeronautical database model shown in Figure 8. It contains three datasets: Context, Coverage_Area, and Obstacles. In the Coverage_Area dataset, it already contains two feature classes that are needed in this project: ADHP and ADHPSurfaceline. Consequently, only the OIS_Spec, FFA_OIS, and eTOD_OIS need to be integrated into the simplified aeronautical database model. 


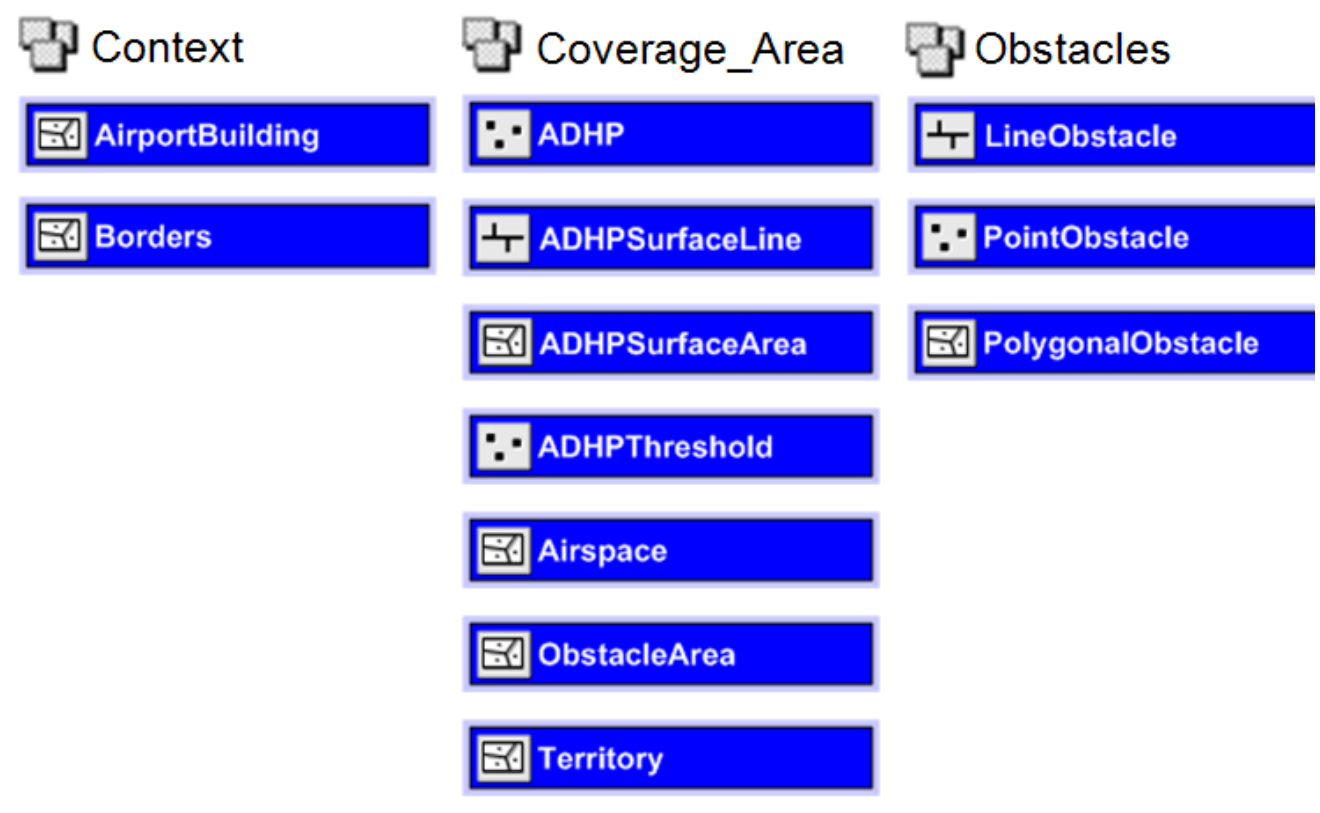

\section{Figure 8: $\quad$ Simplified Aeronautical Database Model For eTOD}

The OIS features are the main product in this project, and are stored in the

ObstacleArea polygon feature class in the simplified aeronautical database model.

However, the FAA OIS and eTOD OIS need to be separated into two feature classes. Therefore, the FAA_OIS and eTOD_OIS feature classes have been added into the Coverage_Area dataset. For the OIS_Spec, it is a reference table and has all the OIS parameters for generating the OIS features. Therefore, it is suitable to be categorized in the Context dataset. Figure 8 provides a visual representation of the updated aeronautical database model.

\subsection{Data Sources}

The data source of the simplified aeronautical database model mentioned in previous sections is from ESRI. Their PLTS - Aeronautical Solution contains extensive models and schemas for digital aeronautical data. The Aeronautical Solution maintains an extensive set of tutorial data and organizes it in a Spatial Data Engine (SDE) database 
called Aeronautical Sample Production (ASPm). This database contains many feature classes, relationship classes, and tables. These data are configured with complicated sets of attributes, domains, and subtypes.

\subsection{Data Scrubbing and Loading}

With the successful creation of the simplified aeronautical database model by David Robinson mentioned in section 4.1, the data for this project can be inherited from this database model. However, due to the three dimensional requirement, there are some feature classes that have to be recreated because of the Z-value being disabled. These feature classes include the ADHPFeatureLine, PointObstacle, LineObstacle, and PolygonObstacle. Three new runway centerlines were drawn between the threshold points to replace the original runway centerlines in order to enable the $\mathrm{Z}$-value in the ADHPFeatureline feature class. For the PointObstacle, LineObstacle, and PolygonObstacle feature classes, they do not contain any features. Therefore, no features needed to be recreated for these feature classes.

\subsection{Summary}

This chapter documented the establishment of the OIS file geodatabase by starting from a conceptual design. It used a non-technical perspective to describe all the necessary components of this project, the characteristics of the components and the relationships between them. The logical design was formulated after the conceptual design. It transformed the concepts into a technical design such as entities, attributes, and types of relationships. This technical design was represented by a UML diagram. The physical design formalized the table names, attribute names, attribute data types, and attribute 
default values. With all the designs settled, adding the discussion of the data source and the data scrubbing is the final step to finish this database design. 


\section{Chapter 5 - Geoprocessing Tool Design}

This chapter describes the details for the OIS GP tool. The first section lists out the essential components in GP. The hierarchy of GP is presented in section 5.2. The foundation of the GP has been set up based upon sections 5.1 and 5.2. In section 5.3, the parameters and decision structure of the OIS GP tool will be described thoroughly.

Finally, this chapter introduces the classes and attributes that will be implemented for the GP tool.

\subsection{Geoprocessing Components}

Geoprocessing has two main components: the GP tool and GP framework. The GP tool can be thought of as an operation that takes an input or set of inputs and generates one or more outputs. Currently there are more than two hundred GP tools in ArcGIS and some of them are most commonly used for GIS analysis, including the buffer tool, the clip tool, the intersect tool, and the split tool. The GP framework is the set of dialog boxes and windows the user employs to manage and execute tools. With the GP framework, users can easily implement their ideas into new software that can be modified and shared with other GIS users. For example, ModelBuilder, a geoprocessing model, allows the user to quickly build a new tool with an easy-to-use visual programming language. Another example is scripting. Script languages, such as Python and Perl, use built-in, high-level functions to create new tools. Unlike other system programming languages such as $\mathrm{C}++$ and Java, scripting languages are easier to learn and use.

In this project, OIS generation is a complex process that includes computing a large amount of arithmetic calculations and rendering new features which involve two 
specifications. Besides model and script, a new GP tool can also be built by using ArcObjects, a set of computer objects specifically designed for programming with the ArcGIS Desktop application. ArcObjects includes feature classes, tables, features, data frames, and so on. ArcObjects was delivered as part of the ArcGIS Software Development Kit (SDK). Application developers use it to build new GIS applications or to extend the functionality for an existing GIS application. In geoprocessing, ArcObjects can be used to build new tools with more complex logic and algorithms. Therefore, ArcObjects is well suited for the OIS GP tool. Because ArcObjects can be used to build more complex tools, it is meant to be used as a system programming language to allow the programmer to access low-level primitive.

C Sharp is a system programming language and is used for implementation in this project. It is disarmingly simple, but highly expressive when it comes to implementing GP. It also includes all the support for structured, component-based, and object-oriented programming. The biggest breakthrough in C Sharp when compared to the traditional object-oriented environment like $\mathrm{C}++$ is the presence of garbage collection. The garbage collector can find objects that are no longer referenced and return their memory to the heap for reuse; there are no memory leaks and hence, this capability reduces the debugging and maintenance time. 


\subsection{Geoprocessing Structure}

This section includes the structure of the GP. The GP structure diagram in Figure 9 shows the tool relationships. It also shows the classes implemented by ESRI and the classes that are provided to extend the GP framework. The GP structure can be divided into four types of tools: GPModelTool, GPFunctionTool, GPCustomTool, and GPScriptTool. (ESRI)

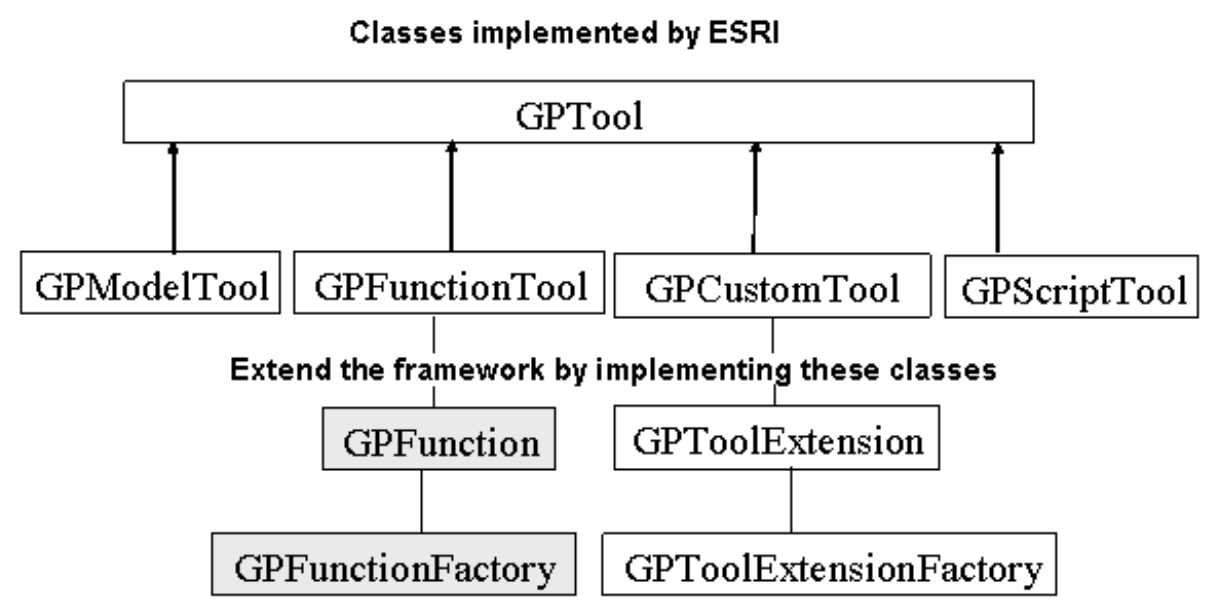

Figure 9: Geoprocessing Structure

The decision to use ArcObjects and C\# for this project makes the OIS GP tool well suited for the GPFunctionTool category. A GP function requires implementation of a minimum of two objects: a GPFunction and a GPFunctionFactory. A GPFunction is a programming-class library that has several properties and methods required for creating a GP function tool. The following table lists each GPFunction property and method, and gives a brief description and explains the usage of those properties and methods in OIS GP tool. (ESRI) (ESRI, Geoprocessing Data Types of Parameters and Environments) 
Table 9. GPFunction Properties and Methods

\begin{tabular}{|c|c|c|}
\hline Name & Description & Usage in geoprocessing tool \\
\hline Name & $\begin{array}{l}\text { A property that returns the } \\
\text { name of the function tool. }\end{array}$ & $\begin{array}{l}\text { The name of OIS GP tool is } \\
\text { "Create OIS." }\end{array}$ \\
\hline DisplayName & $\begin{array}{l}\text { A property that returns the } \\
\text { display name of the function } \\
\text { tool. }\end{array}$ & $\begin{array}{l}\text { The display name of OIS GP tool } \\
\text { is "Create OIS." }\end{array}$ \\
\hline FullName & $\begin{array}{l}\text { A property that returns the } \\
\text { function name object for the } \\
\text { GP function tool. }\end{array}$ & $\begin{array}{l}\text { "ObstructionIdentificationSurface" } \\
\text { is the function name object for the } \\
\text { GP function tool. }\end{array}$ \\
\hline ParameterInfo & $\begin{array}{l}\text { A property that returns the } \\
\text { location where the parameters } \\
\text { of the function tool are } \\
\text { defined. }\end{array}$ & $\begin{array}{l}\text { Initialize the OIS parameter input } \\
\text { information. }\end{array}$ \\
\hline UpdateParameters & $\begin{array}{l}\text { A method to change a } \\
\text { parameter in the tool dialog } \\
\text { box or command line. }\end{array}$ & $\begin{array}{l}\text { Update each surface value after } \\
\text { the user specifies the type of OIS. }\end{array}$ \\
\hline UpdateMessages & $\begin{array}{l}\text { A method for message return } \\
\text { after the internal validation } \\
\text { routine. }\end{array}$ & $\begin{array}{l}\text { No implementation in OIS GP } \\
\text { tool. }\end{array}$ \\
\hline Execute & $\begin{array}{l}\text { A method to launch the GP } \\
\text { tool with the array of } \\
\text { parameter values. }\end{array}$ & $\begin{array}{l}\text { Retrieve the parameter input from } \\
\text { the GP tool form and initialize the } \\
\text { logic modules. }\end{array}$ \\
\hline IsLicensed & $\begin{array}{l}\text { A method to check whether } \\
\text { the GP function tool is } \\
\text { licensed to launch. }\end{array}$ & $\begin{array}{l}\text { Return a true value to indicate that } \\
\text { the tool is licensed. }\end{array}$ \\
\hline HelpFile & $\begin{array}{l}\text { A property that returns the } \\
\text { path to a .chm file, which is } \\
\text { used to describe and explain } \\
\text { the function and its operation. }\end{array}$ & $\begin{array}{l}\text { Return the .chm file to describe } \\
\text { and give details to the OIS } \\
\text { function and its operation. }\end{array}$ \\
\hline HelpContext & $\begin{array}{l}\text { A property that returns a } \\
\text { unique context identifier. }\end{array}$ & $\begin{array}{l}\text { No implementation in OIS GP tool } \\
\text { and return a zero value. }\end{array}$ \\
\hline MetadataFile & $\begin{array}{l}\text { A property that returns the } \\
\text { name of the } . x m l \text { file } \\
\text { containing the default } \\
\text { metadata for this function } \\
\text { object. }\end{array}$ & $\begin{array}{l}\text { No implementation in OIS GP } \\
\text { tool. }\end{array}$ \\
\hline GetRenderer & $\begin{array}{l}\text { A property that is used to set a } \\
\text { custom renderer for the output } \\
\text { of the function tool. }\end{array}$ & $\begin{array}{l}\text { No implementation and return a } \\
\text { null value for OIS GP tool. }\end{array}$ \\
\hline
\end{tabular}


Once the GPFunction tool is formed, a GPFunctionFactory is ready to be designed and implemented in order to provide access to the GPFunction. A function factory is a logical grouping of function tools and is responsible for assigning the function name objects for each function. In most cases, a single Dynamic Link Library (DLL) can contain one function factory with several function tools. The following table lists the properties and methods of GPFunctionFactory. (ESRI) (ESRI, Geoprocessing Data Types of Parameters and Environments)

Table 10. Geoprocessing Function Factory Properties and Methods

\begin{tabular}{|l|l|}
\hline \multicolumn{1}{|c|}{ Name } & \multicolumn{1}{c|}{ Description } \\
\hline CLSID & The class ID of the factory. \\
\hline Name & Name of the function factory. \\
\hline Alias & Alias name of the function factory. \\
\hline GetFunction & $\begin{array}{l}\text { Creates and returns a function object based on the input } \\
\text { name. }\end{array}$ \\
\hline GetFunctionName & $\begin{array}{l}\text { Creates and returns a function name object based on the } \\
\text { input name. }\end{array}$ \\
\hline GetFunctionNames & $\begin{array}{l}\text { Creates and returns an enumeration of function names that } \\
\text { the factory supports. }\end{array}$ \\
\hline GetFunctionEnvironments & $\begin{array}{l}\text { Creates and returns an enumeration of GPEnvironment } \\
\text { object. }\end{array}$ \\
\hline
\end{tabular}

\subsection{OIS Parameters and Decision Structure}

A planned decision structure combined with accurate definitions of parameters can improve the implementation time. In GP, the input parameters are stored in an array; the first parameter being stored in the first position in the array, the second parameter being 
stored in at the second position in the array, and so on. Each parameter has its type, direction, data type, and dependency. Parameter type is required, optional or derived. If the parameter type is declared as required or derived, then the parameter is necessary for the GP tool execution. Parameter direction is either input or output. Derived parameter applies only to output parameter. Parameter data type is the type of input data. Specifying the data type for each parameter is important for the GP internal validation. Common data types in geoproceesing parameter are workspace, feature layer, feature, string, double, list, and table. Parameter dependency is a parameter definition which relies on the value of another parameter. Listing fields in a table is a good example of parameter dependency. When listing fields, the field parameter is always dependent on the input table parameter in order to list the correct fields.

There are a total of fifteen parameters in the OIS GP tool, fourteen input parameters and one output parameter. Input parameters include the Geodatabase, the runway feature class, OIS type, OIS unit, primary surface or area $2 \mathrm{a}$ length, primary surface or area 2a width, horizontal surface height, horizontal surface radius, conical surface or area $2 \mathrm{c}$ offset, conical surface or area $2 \mathrm{c}$ slope, approach surface or area $2 \mathrm{~b}$ slope, approach surface or area $2 b$ length, approach surface extended width or area $2 b$ splayed angle, and transitional surface slope. The only output parameter is the OIS feature class. The following table shows the properties of each parameter. As discussed in Chapter Four, there are several zones that are similar to each other in the OIS specification descriptions from both FAA and ICAO. First is the primary surface and area 2a. Second is the approach surface and area $2 \mathrm{~b}$. Third is the conical surface and area $2 \mathrm{c}$. Based on these similarities, those zones can share one input parameter. 
Table 11. OIS Parameters Properties

\begin{tabular}{|l|l|l|l|l|}
\hline \multicolumn{1}{|c|}{ Name } & \multicolumn{1}{|c|}{ Type } & Direction & Data Type & Dependency \\
\hline Geodatabase & Required & Input & Workspace & No \\
\hline Input Runway & Required & Input & Feature Layer & No \\
\hline OIS Type & Required & Input & String & No \\
\hline OIS Unit & Required & Input & String & No \\
\hline $\begin{array}{l}\text { Primary Surface / Area 2a } \\
\text { Length }\end{array}$ & Required & Input & Double & No \\
\hline $\begin{array}{l}\text { Primary Surface / Area 2a } \\
\text { Width }\end{array}$ & Required & Input & Double & No \\
\hline Horizontal Surface Height & Required & Input & Double & No \\
\hline Horizontal Surface Radius & Required & Input & Double & No \\
\hline $\begin{array}{l}\text { Conical Surface / Area 2c } \\
\text { Offset }\end{array}$ & Required & Input & Double & No \\
\hline $\begin{array}{l}\text { Conical Surface / Area 2c } \\
\text { Slope }\end{array}$ & Required & Input & Double & No \\
\hline $\begin{array}{l}\text { Approach Surface / Area 2b } \\
\text { Length }\end{array}$ & Required & Input & Double & No \\
\hline $\begin{array}{l}\text { Approach Surface Extended } \\
\text { Angle }\end{array}$ & Required $2 \mathrm{~b}$ Splayed & Input & Double & No \\
\hline $\begin{array}{l}\text { Approach Surface / Area } 2 \mathrm{~b} \\
\text { Slope }\end{array}$ & Required & Input & Double & No \\
\hline Transitional Surface Slope & Required & Input & Double & No \\
\hline
\end{tabular}


Although there is no dependency for all the parameters, the sequence of the input parameter is important to the decision structure of the OIS GP tool. For most of the GIS solution, geodatabase input should always be considered as the first parameter because all the output and input data is stored there. Therefore, in this project, the file geodatabase is the first input parameter. The input and output feature classes are the proper logical sequence to the decision structure. After choosing the input and output feature classes, OIS type and OIS unit need to be specified for the input parameter. Finally, the surface parameters are retrieved from the geodatabase and populated automatically based on the previous input. During each input parameter, there is an internal validation to check the parameter properties, such as the type, direction, data type, and dependency. This validation is derived from the beginning of the GP tool parameter design. The following diagram illustrates the decision structure for the OIS GP tool. 


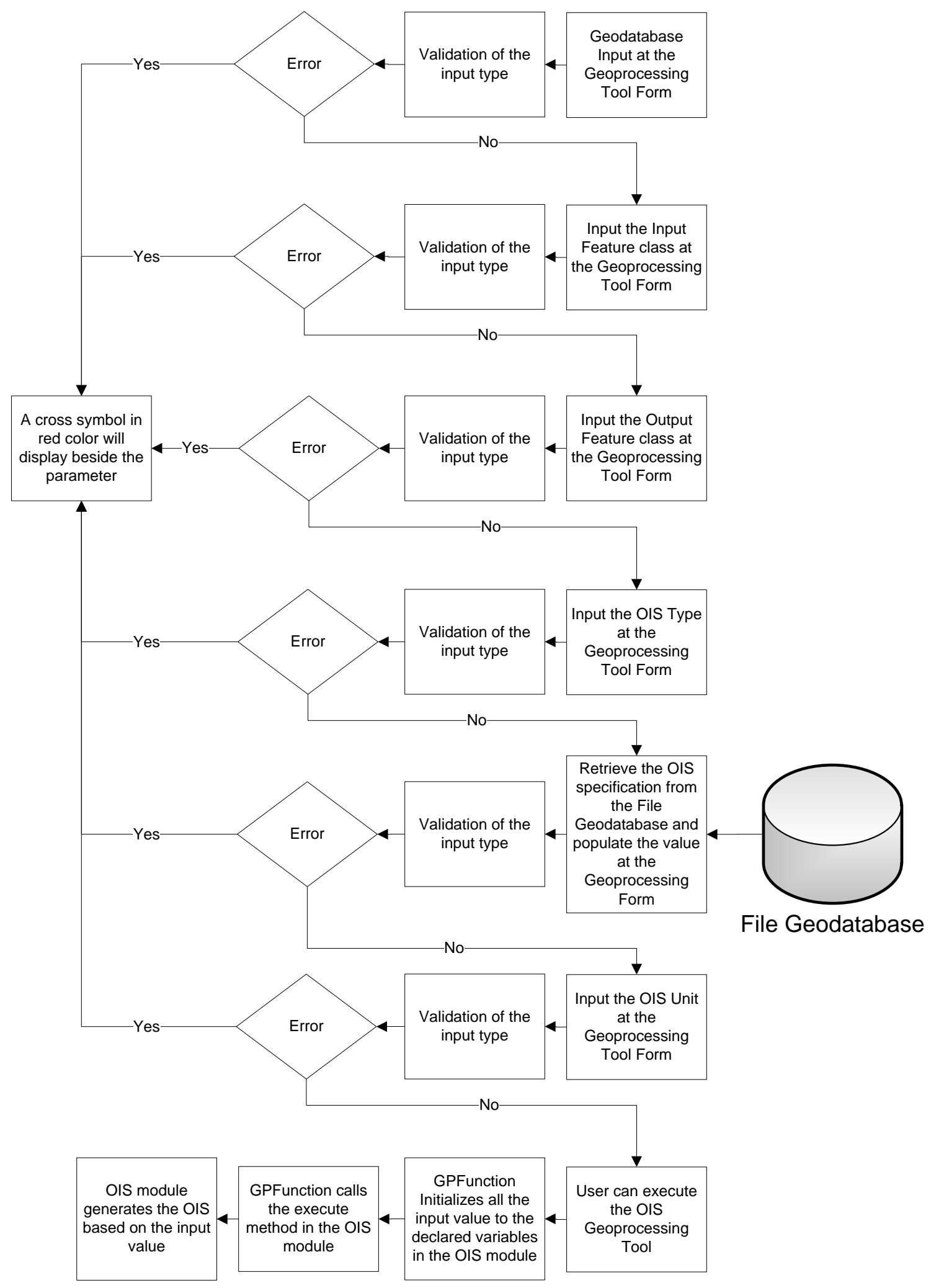

Figure 10: OIS Geoprocessing Tool Decision Structure 


\subsection{Summary}

This chapter first described the key concepts of the GP framework and the fundamental elements of the GP components in order to find the best design of the OIS GP tool and fully utilize the GP function capacity. Second, the GP structure was explored thoroughly to facilitate the GP function factory and GP function tool design. With the above design drafted, the necessary parameters for the OIS GP tools as well as the decision structure for the OIS GP have been formulated. This chapter, combined with the previous chapter, forms a solid design foundation. The next chapter starts implementing the actual GP tool with this concrete foundation. 



\section{Chapter 6 - Implementation}

This section depicts the steps taken to complete this project. After the design was completed and the necessary data were organized, the project moved to the next stage implementation. It was during this phase that a GIS application tool was developed.

This chapter concentrates on the efforts to implement a GP tool. This tool is used to generate the OIS for an airport runway. Section 6.1 discusses the implementation of OIS GP function factory. Section 6.2 explains the OIS GP function along with all its properties and methods. Section 6.3 describes the detail of the logic module. The last section summarizes this chapter.

\subsection{OIS Geoprocessing Function Factory}

The GP function factory is called OISGPFunctionFactory and is written in C Sharp. It contains three properties: Alias, CLSID, and Name. The purpose of these properties is retrieving information rather than storing information. The general structure is as follows:

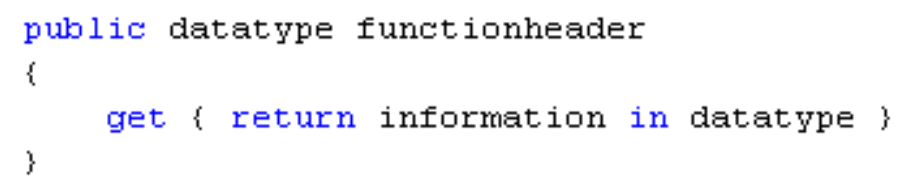

Figure 11: Function Factory Property General Structure

Besides the properties, there are seven methods implemented inside the OISGPFunctionFactory: Reg, Unreg, GetFunctionNames, CreateGPFunctionName, GetFunction, GetFunctionName, and GetFunctionEnvironment. Reg and UnReg methods are used to register the function factory with the ESRI geoprocessor function factory component category. The CreateGPFunctionNames method initializes GP function information such as category, description, name, and display name. GetFunction method 
is used to retrieve the actual GP function pointer. GetFunctionName and

GetFunctionNames methods are to retrieve a GP function name and multiple GP function names respectively. All GetFunction, GetFunctionName, and CreateGPFunctionNames methods use switch case method to return the correct function and its name. The general structure is shown in Figure 11. The GetFunctionEnvironment method creates and returns an enumeration of GP environment objects. In this GP function factory, there is no new environment setting created and consequently, this method returns a null value.

After having explained the implementation of methods and properties inside the GP function factory, the next section will discuss the actual OIS GP function implementation.

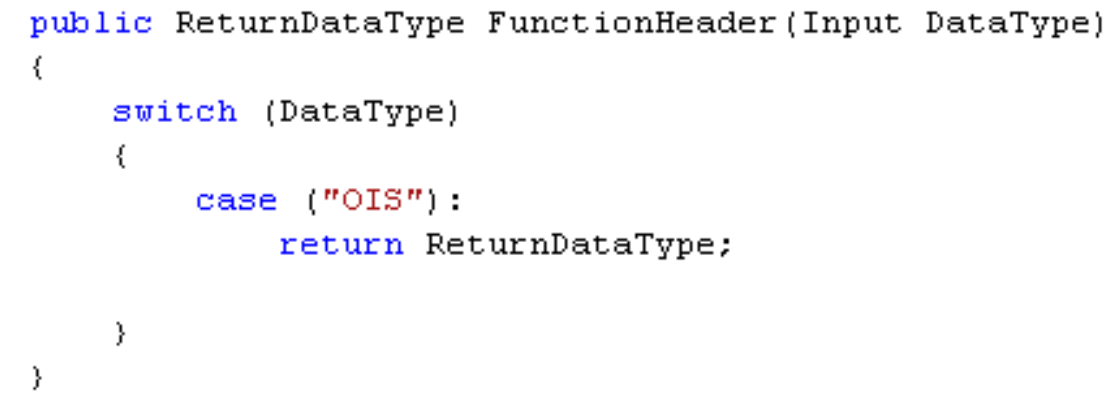

Figure 12: Function Factory Method General Structure

\subsection{OIS Geoprocessing Function}

The OIS GP function module is called OISGP. A GP function module often contains all the properties and methods listed in the previous chapter as well as having all the OISGP module properties and methods functions header declaration. These include the OISGP constructor, isLicensed, UpdateParameters, UpdateMessages, FullName, Name, DisplayName, ParameterInfo, Validate, MetadataFile, GetRender, HelpFile, HelpContext, and Execute. Nevertheless, not all the properties and methods necessarily 
need to be implemented for OIS GP tool. Consequently, this section will discuss the functions that will actually be implemented.

The constructor function is vital for a class. In OISGP module, the constructor initializes an internal class called GPUtilities. GPUtilities, a helper class for geoprocessing, provides functions to unpack the GP parameters value and call the internal validation methods. The ParameterInfo function declares the GP parameters. In the previous chapter, some properties for each parameter were summarized in table 10 . The following tables list additional declarations for each parameter.

The parameter name indicates the actual name used in the program code. Some abbreviations for the parameter name need to be clarified. For example, input_ps_length refers to input primary surface length. Another example is input_as_slope, which refers to the input slope of approach surface. The data type class describes the actual GP data type class name. Similarly, the data value class shows the actual GP data value class name. The domain column specifies the parameter domain and the data value added into its domain. The display string in GP tool form depicts the description of the parameter. 
Table 12. OIS Parameters Declarations

\begin{tabular}{|l|l|l|}
\hline \multicolumn{1}{|c|}{ Parameter Name } & \multicolumn{1}{|c|}{ Data Type Class } & \multicolumn{1}{c|}{ Data Value Class } \\
\hline input_geodatabase & DEWorkspaceTypeClass & DEWorkspaceClass \\
\hline input_features & GPFeatureLayerTypeClass & GPFeatureLayerClass \\
\hline output_features & GPFeatureLayerTypeClass & GPFeatureLayerClass \\
\hline input_units_Type & GPStringTypeClass & GPStringClass \\
\hline input_Specification_Type & GPStringTypeClass & GPStringClass \\
\hline input_ps_length & GPDoubleTypeClass & GPDoubleClass \\
\hline input_ps_width & GPDoubleTypeClass & GPDoubleClass \\
\hline input_hs_radius & GPDoubleTypeClass & GPDoubleClass \\
\hline input_hs_height & GPDoubleTypeClass & GPDoubleClass \\
\hline input_cs_slope & GPDoubleTypeClass & GPDoubleClass \\
\hline input_cs_offset & GPDoubleTypeClass & GPDoubleClass \\
\hline input_as_length & GPDoubleTypeClass & GPDoubleClass \\
\hline input_as_slope & GPDoubleTypeClass & GPDoubleClass \\
\hline input_as_extendedwidth & GPDoubleTypeClass & GPDoubleClass \\
\hline input_ts_slope & GPDoubleClass \\
\hline
\end{tabular}


Table 13. Additional OIS Parameters Declarations

\begin{tabular}{|c|c|c|}
\hline Parameter Name & Domain & $\begin{array}{l}\text { Display String in } \\
\text { Geoprocessing Tool Form }\end{array}$ \\
\hline input_geodatabase & N/A & $\begin{array}{l}\text { "Please Select a } \\
\text { Personal/SDE/Geo Database } \\
\text { Express Database" }\end{array}$ \\
\hline input_features & N/A & "Input Feature Class" \\
\hline output_features & N/A & "Output Feature Class" \\
\hline input_units_Type & $\begin{array}{l}\text { GPCodedValueDomainClass } \\
\text { The strings added to this } \\
\text { domain are "Meters" and } \\
\text { "Feet" }\end{array}$ & "Input Units" \\
\hline input_Specification_Type & $\begin{array}{l}\text { GPCodedValueDomainClass } \\
\text { The strings added to this } \\
\text { domain are "FAA" and } \\
\text { "eTOD" }\end{array}$ & $\begin{array}{l}\text { "Obstruction Identification } \\
\text { Surface Type (eTOD or } \\
\text { FAA)" }\end{array}$ \\
\hline input_ps_length & N/A & "Primary Surface Length" \\
\hline input_ps_width & N/A & $\begin{array}{l}\text { "FAA: Primary Surface } \\
\text { Width eTOD: Area 2a } \\
\text { Width" }\end{array}$ \\
\hline input_hs_radius & N/A & "Horizontal Surface Radius" \\
\hline input_hs_height & N/A & "Horizontal Surface Height" \\
\hline input_cs_slope & N/A & $\begin{array}{l}\text { "Conical Surface Slope } \\
\text { eTOD: Area 2c Slope" }\end{array}$ \\
\hline input_cs_offset & N/A & $\begin{array}{l}\text { "Conical Surface Offset } \\
\text { eTOD: Area 2c Offset" }\end{array}$ \\
\hline input_as_length & N/A & $\begin{array}{l}\text { "FAA: Approach Surface } \\
\text { Length eTOD: Area 2b } \\
\text { Length" }\end{array}$ \\
\hline input_as_slope & N/A & $\begin{array}{l}\text { "FAA: Approach Surface } \\
\text { Slope eTOD: Area 2b } \\
\text { Slope" }\end{array}$ \\
\hline input_as_extendedwidth & N/A & $\begin{array}{l}\text { "FAA: Approach Surface } \\
\text { Extended Width eTOD: } \\
\text { Area 2b Splayed Angle" }\end{array}$ \\
\hline
\end{tabular}




\begin{tabular}{|l|l|l|}
\hline input_ts_slope & N/A & "Transitional Surface Slope" \\
\hline
\end{tabular}

The UpdateParameters function updates all parameter display values and calls the internal validation to check whether the input data type is matched to the parameter declaration in ParameterInfo function. To update a parameter, the GPParameterEdit class is first used to extract the parameter value from the parameter array. Next, it casts the retrieved value to an element in GPParameter class. Then, it sets the desired value to the GPParameter element in order to complete the update process. For instance, all the surface input value will be updated once the user chooses the OIS specification type. The UpdateParameters function will get called every time a parameter value is changed. To avoid redundancy in the process and to improve performance, two properties are implemented to prevent the program cursor from running the code to process the operation redundantly. The first property is called IsEmpty. It indicates whether the value of a parameter is empty or not. If the value of a parameter is empty, then the program cursor will skip the operation for that particular parameter. This also helps the program cursor catch potentially uninitialized pointers which might otherwise crash the application. The second property is called Alter. It returns a Boolean value to specify the change of a parameter value. If the Alter property returns false, then the program cursor will not continue the following scope so that the codes inside that scope will not get processed.

The major task of Execute function is to launch the GP tool. To elaborate this task, it first retrieves and validates the finalized input values from the GP parameter array. Next it creates the new objects for the logic modules and initializes them. Then it passes 
the parameter values to the logic objects. Finally it calls the execution method from the logic objects.

\subsection{OIS Parameters and OIS Creation modules}

Geoprocessing function factory module and GP function module form the user interface and allow users to input the OIS measurement values. After implementing these two modules, two more modules are needed to complete the OIS generation described in this section.

The first module is the OIS parameter module. OIS parameter module acts as a property class to store and retrieve the temporary OIS measurement values, including the length, height, and angle. It is written in C Sharp programming language. The general structure of this module is demonstrated in the following figure.

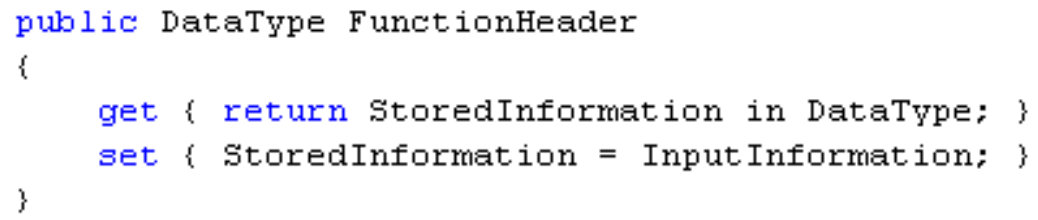

\section{Figure 13: General Structure of OIS Parameter Module}

The second module is the actual logic to construct the OIS feature. It contains ten methods including execute, getPrimarySurface, getApproachSurface, getHorizontalAndConicalSurface, getTransitionalSurface, getIntersectionPoint, constructSurfaceArc, createOISFeature, MakeZAware, and UnitConversion.

The execute method is called from the GP module to indicate that all the input values have been validated and stored in the OIS parameter module. In addition, it checks the existence of the runway feature layer in the table of contents and the selected runway feature. Also it directs which surface is going to be generated based on the specification, 
either FAA or eTOD. The execute method takes the IGPMessage as the input parameter to display the GP message in the GP dialog during the GP function execution and returns a Boolean value to indicate the result of the execution. The function header is presented in Figure 14,

public Boolean execute (IGPMessages gpMessages)

\section{Figure 14: Execute Method Function Header}

getPrimarySurface method generates the primary surface regardless of specification. The primary surface is a rectangular shaped surface and acts as the base of the whole OIS structure because other surfaces are formed around it. getPrimarySurface method takes the input of the runway from point, the runway to point, length of the surface, depth of the surface, and a Boolean value to indicate the primary surface generation and then returns a polyline feature that is rectangular in shape. The function header is shown in the following figure.

\section{private IPolyline getPrimarysurface(IPoint fromPoint, IPoint toPoint, double pslength, double pswidth, bool binvokedForPS)}

\section{Figure 15: Primary Surface Method Function Header}

There are two additional roles for the getPrimarySurface method. The first role is to find the lowest elevation. The OIS is generated based on the lowest elevation because this allows it to catch the most obstacles inside the OIS boundary. The second role is to prepare the reference points for other surfaces, such as approach surface, conical surface, horizontal surface and transitional surface in FAA specification.

getApproachSurface method generates the approach surface for both FAA and eTOD. The approach surface starts from the end of the primary surface and extends 
upward and outward. getApproachSurface method takes the input of the runway from point, runway to point, four approach surface points, primary surface length, primary surface width, approach surface length, approach surface extended width, and approach surface slope. It returns a Boolean parameter to indicate the result of the surface generation. The function header is as follows:

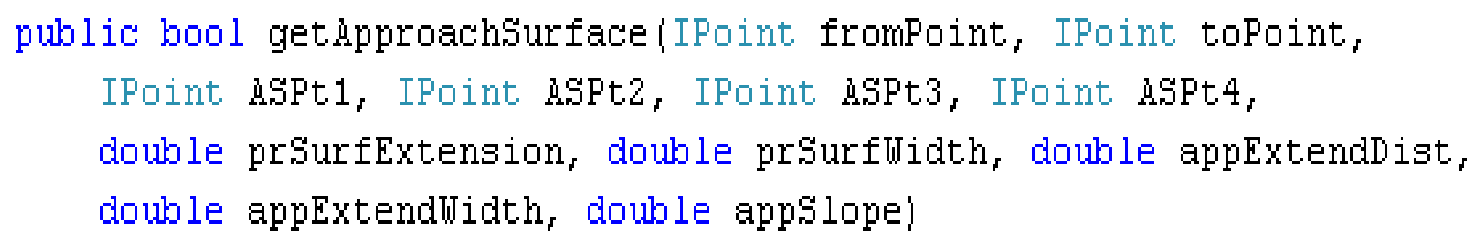

\section{Figure 16: Approach Surface Method Function Header}

The getApproachSurface method calls the getPrimarySurface method with the length that is equal to the primary surface length plus the approach surface length and the width that is equal to the approach surface extended width. Then getPrimarySurface method takes these input values and returns a bigger rectangular polyline feature. Four approach surface points can be obtained from each corner of the bigger rectangular polyline feature. With these four approach surface points, combined with the input approach surface points, ASPt1, ASPt2, ASPt3, and ASPt4, the approach surfaces can be generated. The following diagram illustrates the rectangular shapes and the approach surfaces. The two red rectangular shapes are generated by the getPrimarySurface method and the green polygons are the approach surfaces.

getHorizontalAndConicalSurface method combines the logic for the horizontal surface and conical surface since both surfaces have an outer boundary formed with two arcs and one segment. Also the outer boundary of the horizontal surface is the majority of the conical surface inner boundary. Therefore, combining the logic for both surfaces in 
one method is appropriate and avoids redundant coding. The getHorizontalAndConicalSurface method contains seven input parameters, arcFromPt1, arcToPt1, arcFromPt2, arcToPt2, arcIsCCW, arcCentralAngle, and invokedForSurf. It returns a polyline feature for the output parameter. The function header is as follows:

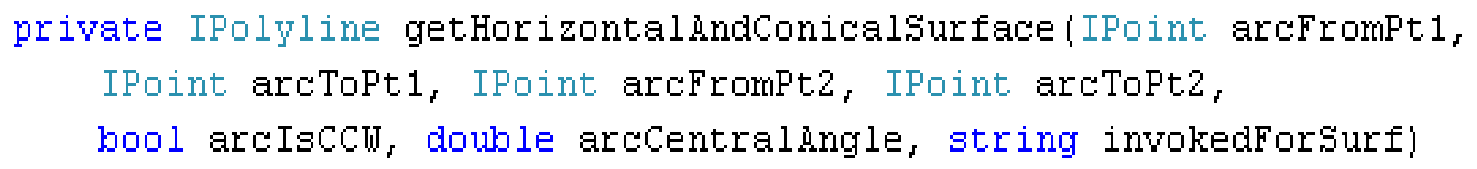

\section{Figure 17: Horizontal and Conical Surface Method Function Header}

The arcFromPt1, arcToPt1, arcFromPt2, arcToPt2 parameters are prepared in the getPrimarySurface method. The getHorizontalAndConicalSurface method uses these input points to generate a football stadium shaped polyline feature for later use in the getIntersectionPoint method. The Boolean value arcIsCCW indicates the arc is constructed counter-clockwise. arcCentralAngle is the angle of the arc and invokedForSurf is a string value to distinguish between the horizontal and the conical surfaces. For eTOD specification, the horizontal surface does not exist and consequently, it only generates the conical surface.

getTransitionalSurface method is the last method for the OIS surface generation. It generates several transitional surfaces to connect all other surfaces in order to form a complete OIS. The getTransitionalSurface method function header can be found in Figure 18. It takes the input of four points that are prepared in the getPrimarySurface method and returns a Boolean value to indicate the result of this method.

public bool getTransitionalsurface(IPoint Tspt1,
IPoint Tspt2, IPoint Tspt3, IPoint TSpt4)

Figure 18: Transitional Surface Method Function Header 
In getTransitionalSurface method, there are four vertical transitional surfaces generated between approach surface, horizontal surface and conical surface for FAA specification. However, these vertical surfaces create a display issue and affect the analysis. The root of this problem lies in the fact that the ArcGIS application cannot identify which side faces outward when a polygon feature is vertical. As a result of this limitation, these vertical surfaces have to be slightly distorted so that the ArcGIS application can distinguish which side of a polygon faces outward.

getIntersectionPoint method is a helper function to find out the point intersected between surfaces. It takes the input of two points, segment index and height. This method first constructs a segment based on two input points. Next it retrieves another segment with the input segment index and then compares it to the newly constructed segment. The method for the comparison is called "Intersect" in ITopologicalOperator class. If the segments intersect each other, the intersect method will return a point geometry. Figure 19 shows the getIntersectionPoint method function header.

$$
\begin{aligned}
& \text { private IGeometry get IntersectionPoint (IPoint pt1, } \\
& \text { IPoint pt2, int isegmentnumber, double height) }
\end{aligned}
$$

\section{Figure 19: Get Intersection Point Method Function Header}

constructSurfaceArc method is another helper function. It constructs the arc for the horizontal surface and conical surface. There are two methods to construct the arc, ConstructEndPointsRadius and ConstructEndPointsAngle. ConstructEndPointsRadius method constructs a circular arc given a start point, an end point, the desired radius, and two Boolean values to indicate the arc is a minor arc constructed in a counter clockwise direction. ConstructEndPointsAngle method constructs a circular arc given a start point, 
an end point, the desired orientation, and the desired central angle. The central angle is measured in radians. After the circular arc is constructed, vertexes are added to the constructed arc by "Densify" method for the analysis purpose. The discussion of "Densify" method can be found in the following chapter. Figure 20 shows the function header of constructSurfaceArc method.

$$
\begin{aligned}
& \text { private Isegment construetsuracedre (IPoint pist, IPoint pTst, } \\
& \text { IPoint puret, int isegmentwuber, bool bFlip, double height) }
\end{aligned}
$$

\section{Figure 20: Construct Surface Arc Method Function Header}

MakeZAware method is to toggle the $\mathrm{Z}$ value to a desired geometry. The input parameters for this method are the geometry object and a Boolean value to toggle the $\mathrm{Z}$ value. The function header of MakeZAware method is shown in the following figure.

$$
\text { public void Makezdnare (IGeometry pGeom, bool binare' }
$$

\section{Figure 21: Make Z Aware Method Function Header}

The last method to discuss is the UnitConversion method. This method converts a value from one unit of measurement to another. There are three input parameters in UnitConversion Method, unitValue, inUnit, and OutUnit. This method returns a value in double data type. The following is the function header of UnitConversion method.

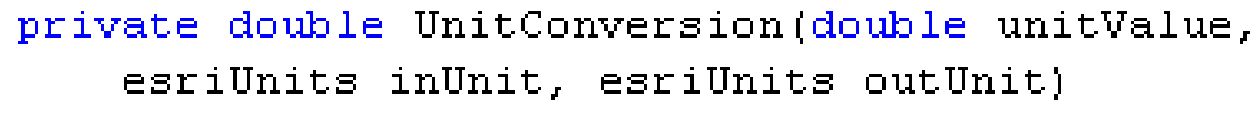

\section{Figure 22: Unit Conversion Method Function Header}




\subsection{Summary}

This chapter discussed the implementation of the OIS GP tool. Also this chapter described the $\mathrm{Z}$ value enable method, polyline usage, intersection point searching, and vertical plane consideration. With this OIS GP tool successfully generated, it is appropriate to move on to the next chapter - Results and Analysis. 


\section{Chapter 7 - Results and Analysis}

The OIS GP tool in the ArcGIS application created for this project provides the aviation industry with the ability to generate the OIS in an effective way and to create a visualization in the ArcGIS application. This chapter reviews the output generated by the OIS GP tool and displays the output in both two-dimensional and three-dimensional views. In addition, the characteristics of both FAA and eTOD OIS and the differences between them will be explained.

\subsection{OIS in Two-Dimensional View}

This section displays the OIS in a two-dimensional view in ArcMap. In order to make the output stand out more, there are three feature layers added in the ArcMap: the runway, airport surface area, and OIS. In Figure 23, the red lines show the runway feature; there are three runways in Salinas Airport. The light orange polygons represent the Salinas Airport surface area. The green lines indicate the OIS for Salinas Airport. The primary surface is a rectangle centered on the runway centerline, beginning on one side of a runway threshold and extending to the opposite runway threshold. The horizontal surfaces are two half-moon shaped planes, the perimeters of which are constructed by swinging arcs of specified radii from the center of each end of the primary surface of each runway. The shapes of approach surfaces are trapezoidal and start from the edge of the primary surface with its width and extend the width to a certain distance with a slope angle. The conical surfaces are two racetrack strip shapes, extending upward and outward from the horizontal surfaces to a certain distance with a slope angle. Transitional surfaces are the connecting surfaces to the above surfaces. There are several transitional surfaces in the FAA OIS, and they can be divided into two types. The first type of transitional 
surface is connected between the primary surface and the horizontal surface. The second type of transitional surface is connected between approach surface, conical surface, and horizontal surface.

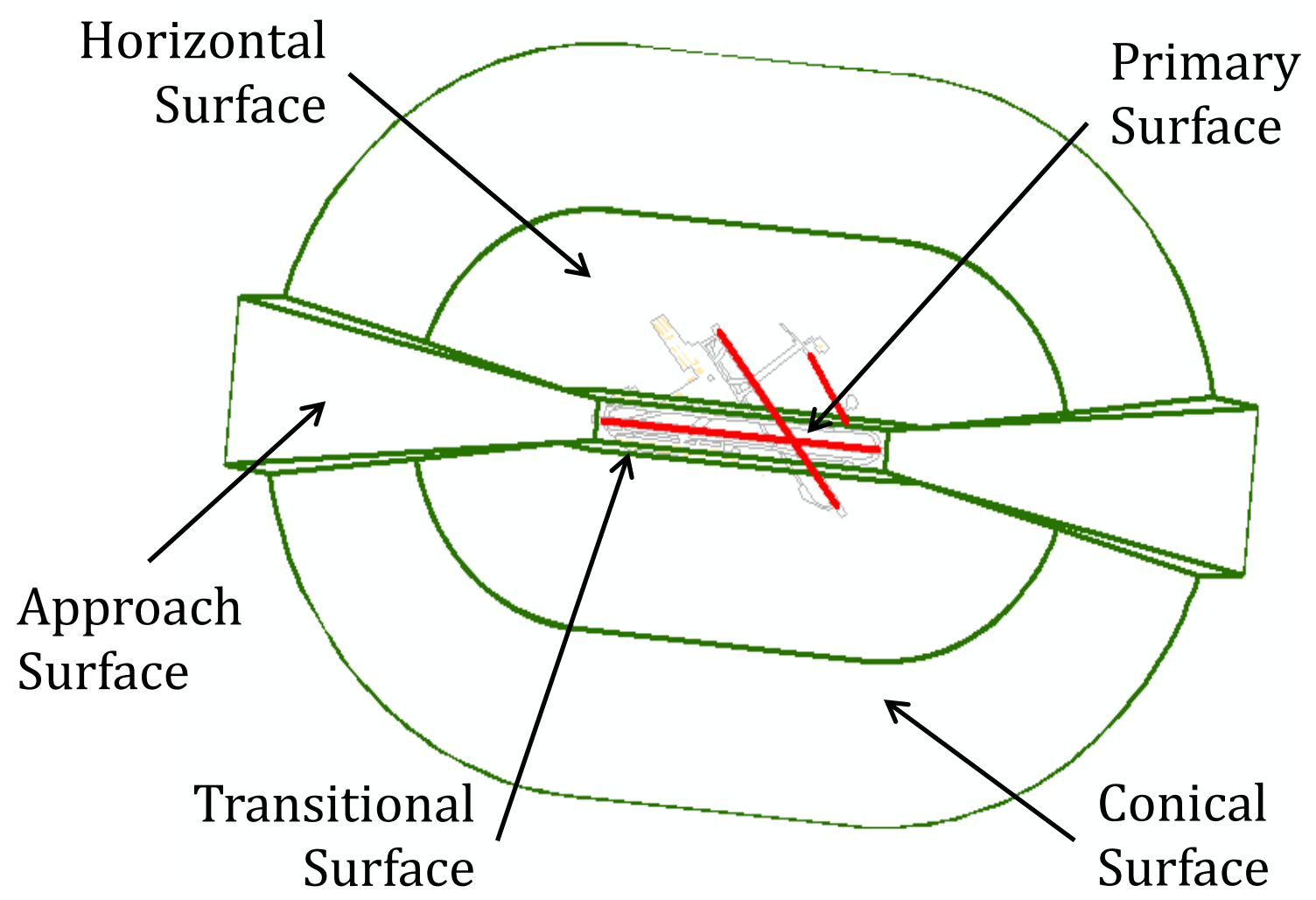

\section{Figure 23: FAA OIS in Two-Dimensional View}

Figure 24 illustrates the eTOD OIS. The red lines show the Salinas Airport runway. The light orange polygons specify the Salinas Airport surface area and the green lines display the eTOD OIS. Area 2a in the blue box has been exaggerated for displaying in a clear rectangular shape. It surrounds the airport runway centerline. Area $2 \mathrm{~b}$, similar to the approach surface in the FAA OIS, has a trapezoidal shape and also starts from the edge of the primary surface with its width and extends the width to a certain distance with a slope angle. Area $2 \mathrm{c}$ is like a hand-held fan, it extends upward and outward from the horizontal surfaces to a certain distance with a slope angle the same as the Area 2b. 


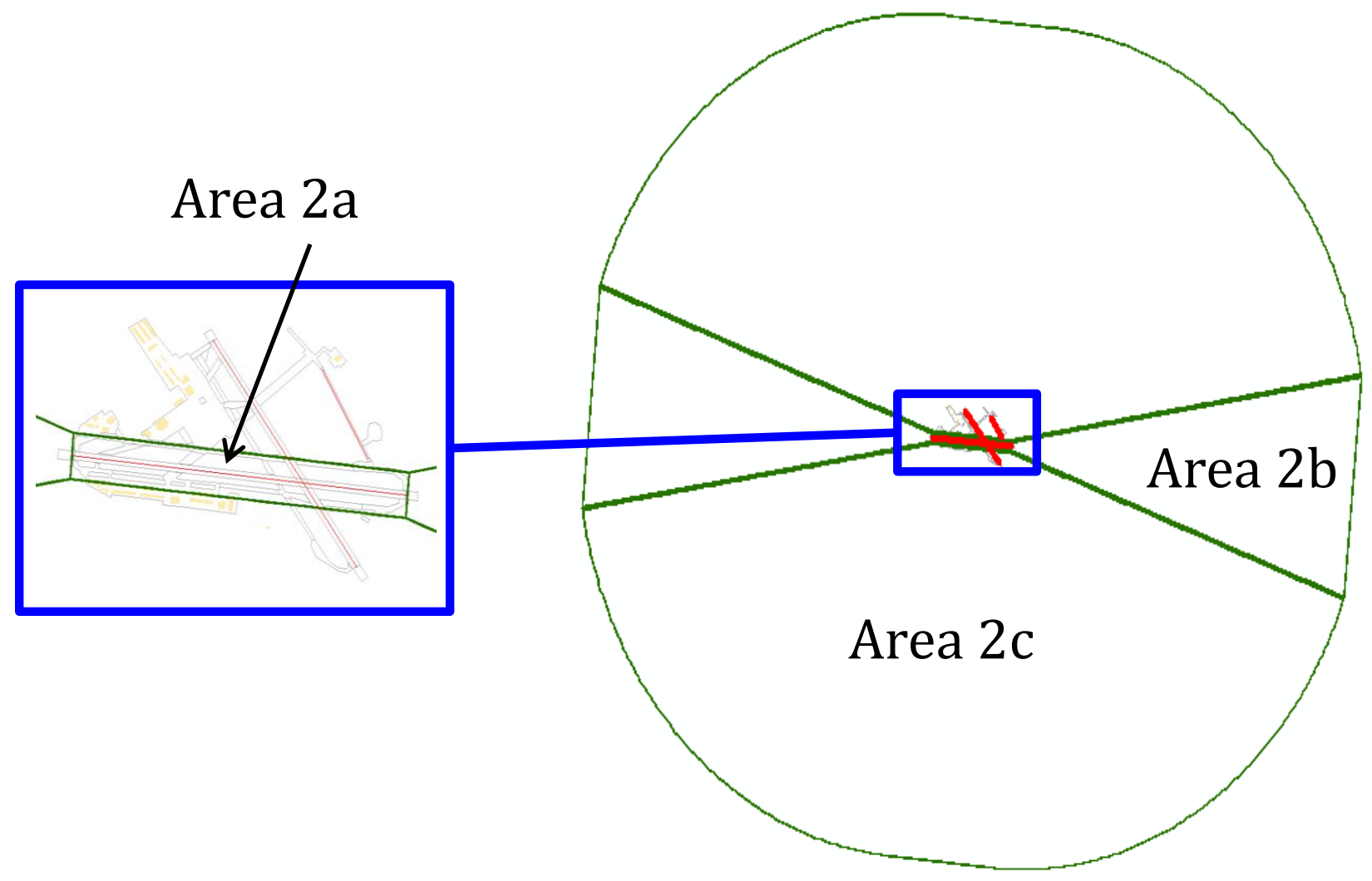

Figure 24: eTOD OIS in Two-Dimensional View

After describing the OIS features generated by the OIS GP tool, putting both OIS side by side is one of the easiest methods to further explore the characteristics for both the FAA and eTOD OIS and the differences between them. In Figure 25, the left diagram indicates that the FAA OIS contains one primary surface, two approach surfaces, two horizontal surfaces, two conical surfaces, and six transitional surfaces. The right diagram shows eTOD OIS has one Area 2a surface, two Area 2b surfaces, and two Area 2c surfaces. Based on these numerical values, the FAA OIS has more surfaces than eTOD OIS. 


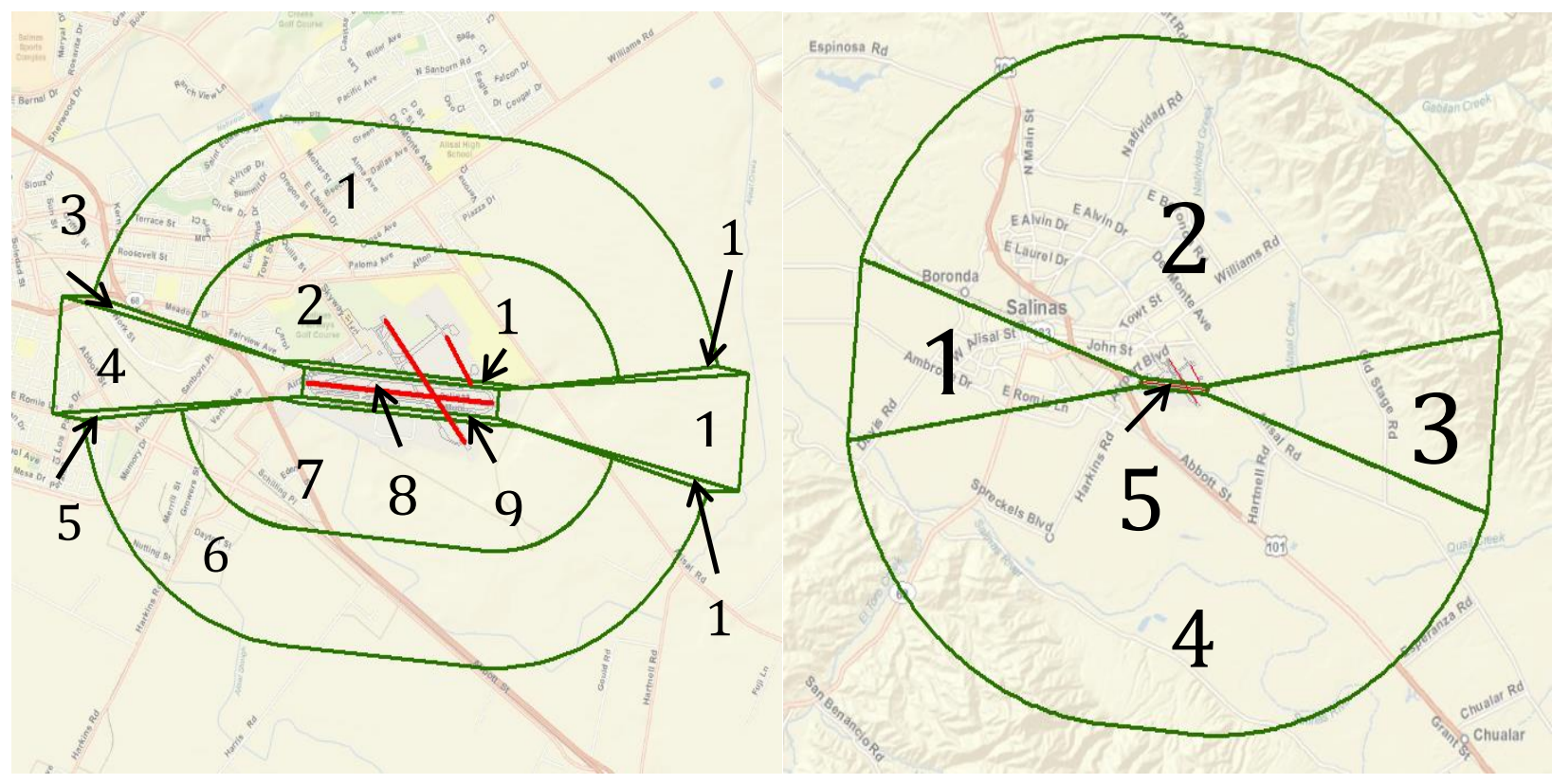

Figure 25: Number of Surfaces for FAA and eTOD

For display purposes, the scale of the left diagram is larger than that of the right diagram in Figure 26. After changing the scale of the left diagram to be the same as that of the right diagram and putting a street layer underneath the runways and OIS features, the size of both the FAA OIS and eTOD OIS can be easily examined. In Figure 26, both OIS are in scale 1:150,000. It clearly shows that the size of eTOD OIS covers a larger area than the FAA OIS. 


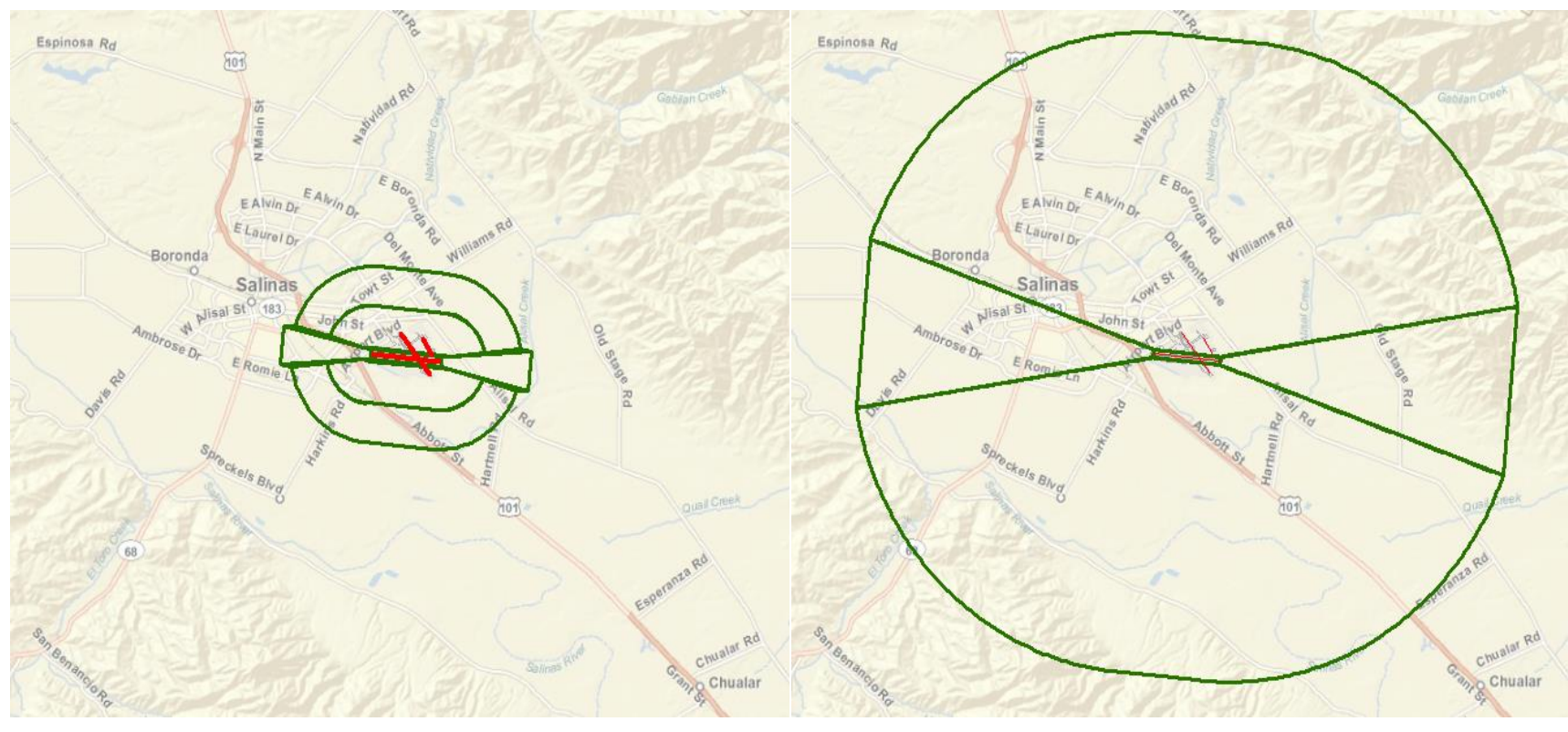

Figure 26: Covered Area for FAA and eTOD

Besides the visual display, the attribute tables for both OIS can also display a considerable amount of information. In Figure 27, the FAA OIS attribute table specifies numerous important pieces of information such as the airport name (ADHP), the runway number (ADHPSurfaceLine), and the type of OIS (OIS_Type).

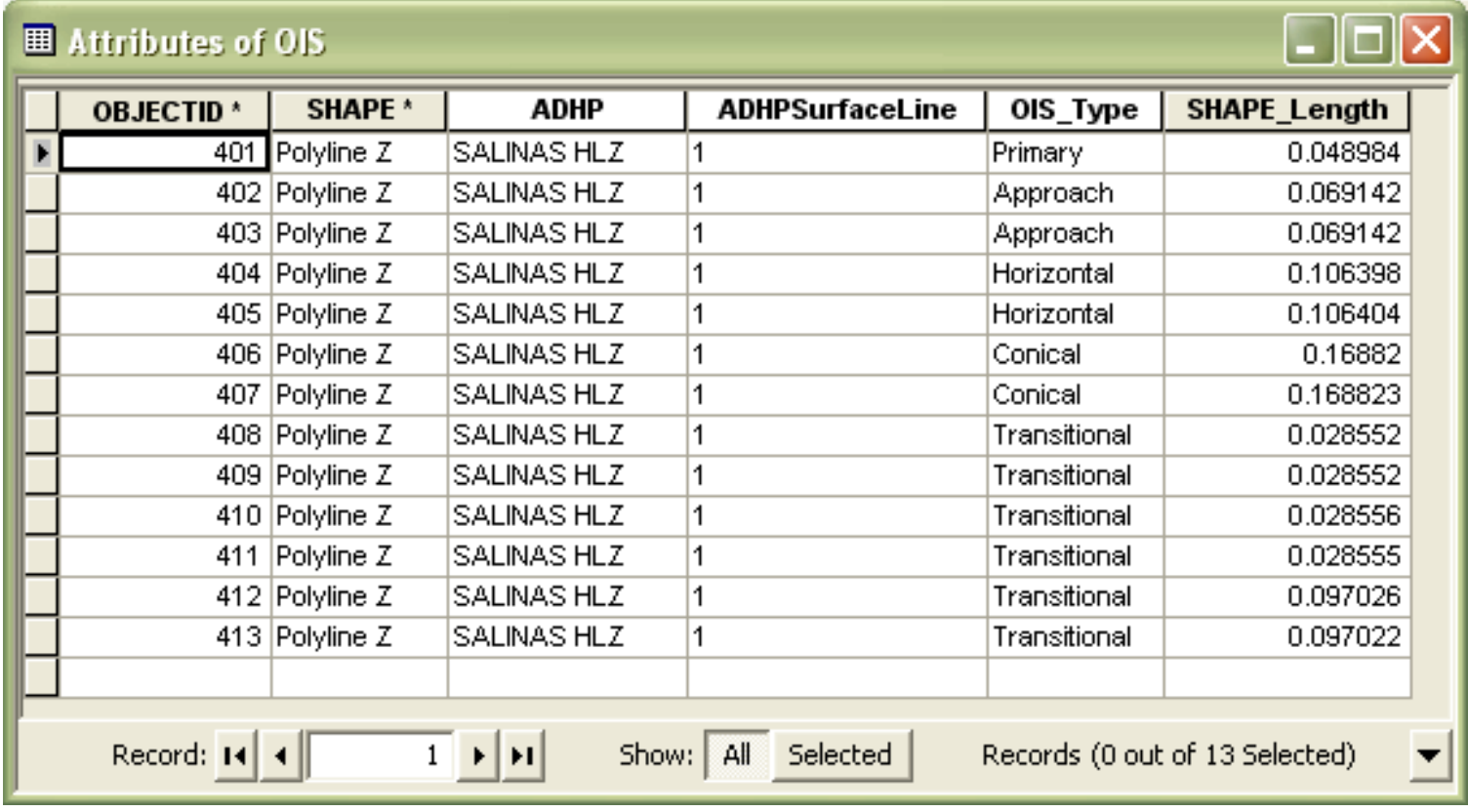

Figure 27: FAA OIS Attribute Table 
Similar to the FAA OIS attribute table, the eTOD OIS attribute table shown in Figure 28 also contains the airport name (ADHP), the runway number (ADHPSurfaceLine), and the type of Area2 (Area2). Both the FAA and eTOD OIS attribute tables indicate the shape of OIS contains a $\mathrm{Z}$ value which is equal to the elevation of the OIS. Because of this characteristic, further results and analysis can be observed in a three-dimensional view.

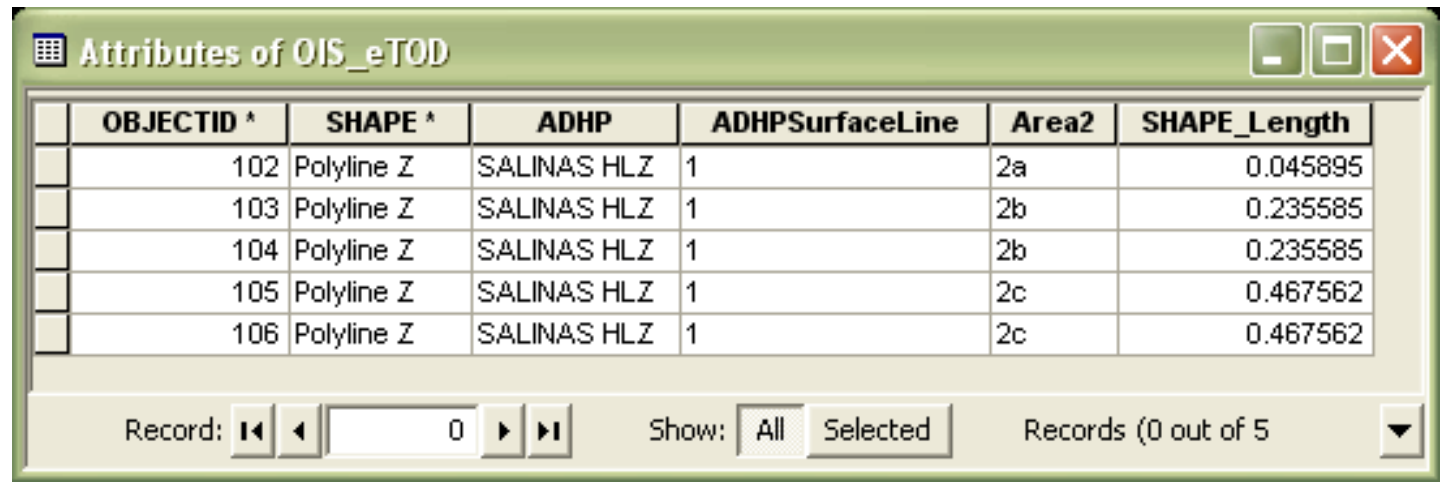

Figure 28: eTOD OIS Attribute Table

\subsection{OIS in Three-Dimensional View}

ArcGlobe is an application well suited for displaying three-dimensional models for the purpose of fully understanding the OIS in a three-dimensional view. By adding the FAA_OIS feature class or eTOD_OIS feature class in the ArcGlobe, exaggerating the elevation conversion factor by 10, the results are simply displayed in Figures 29 and 30. (Kennedy H. K., 2009, pp. 123-142). 


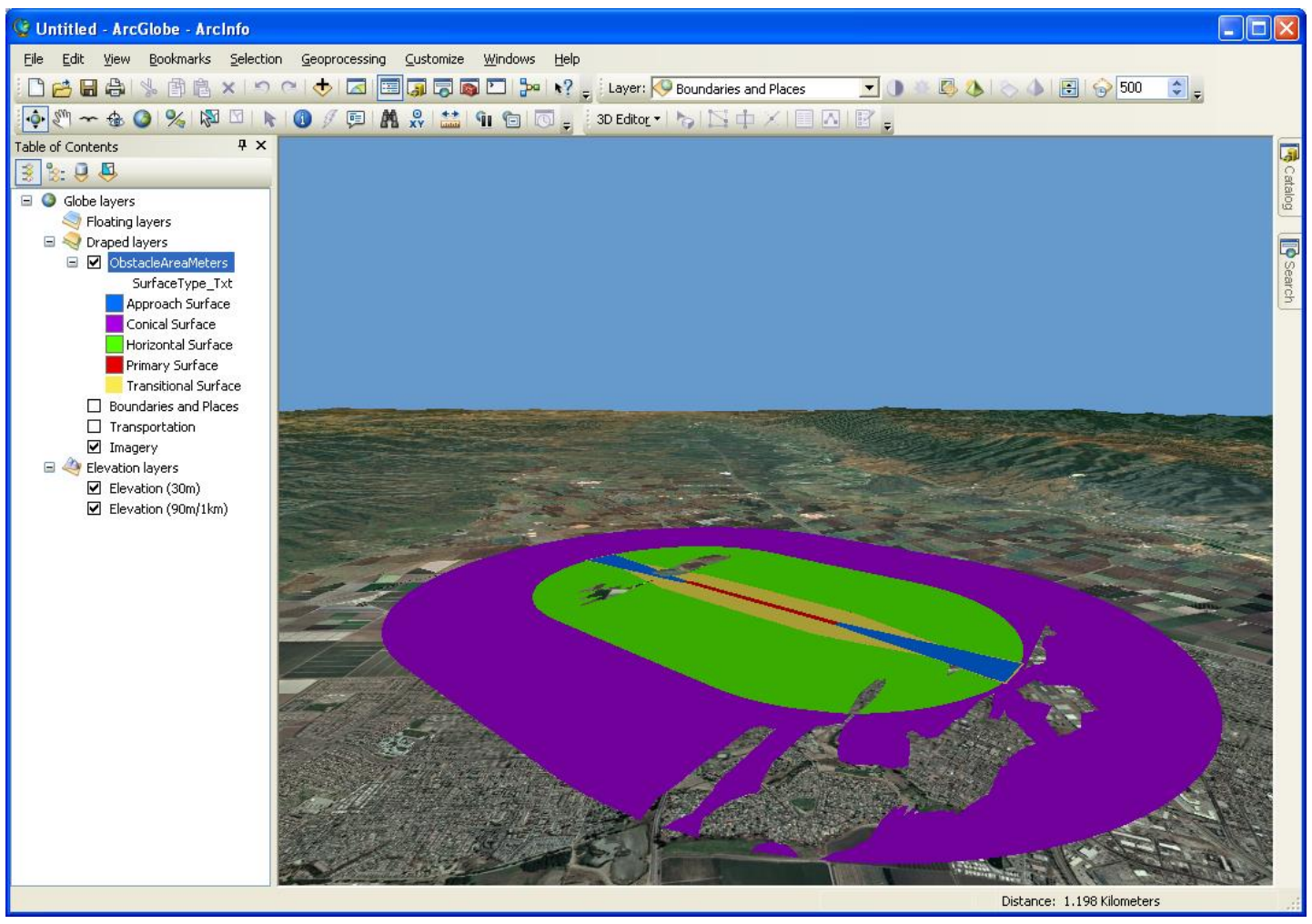

\section{Figure 29: FAA OIS in Three-Dimensional View}

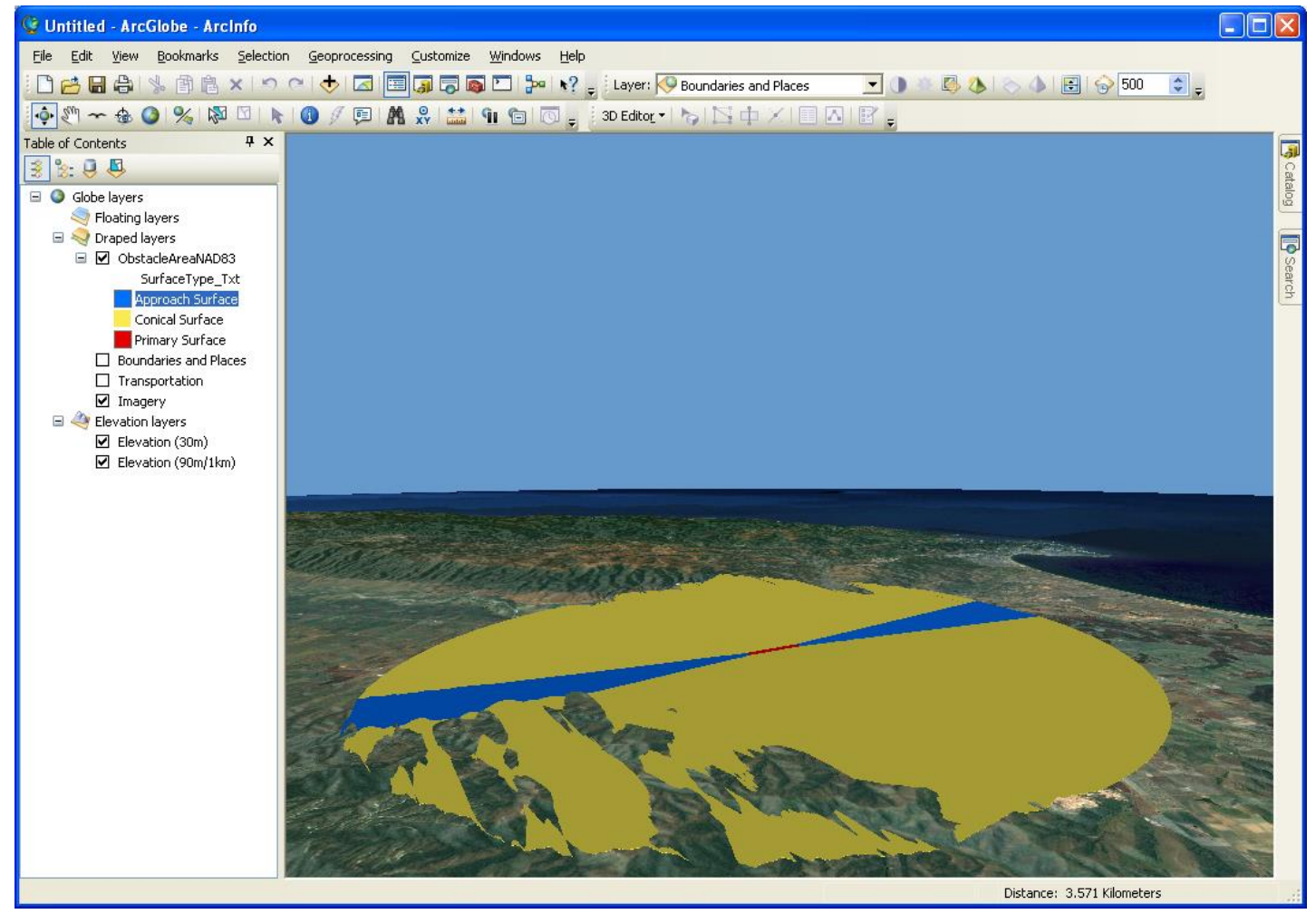

Figure 30: eTOD OIS in Three-Dimensional View 


\subsection{OIS TIN Surfaces}

Another way to explore OIS in a three-dimensional environment is to create Triangulated Irregular Network (TIN) surfaces for the generated OIS features. Figure 31 and Figure 32 show the original TIN surfaces created by the OIS features. Because the slopes of these surfaces are small and the area of these surfaces is relatively large, these two surfaces do not appear to contain any elevation value. For example, the approach surface in FAA OIS is 10,000 feet long but the slope is only $5 \%$. Likewise, the area $2 \mathrm{~b}$ in eTOD OIS is 10,000 meters but the slope is only $1.2 \%$.

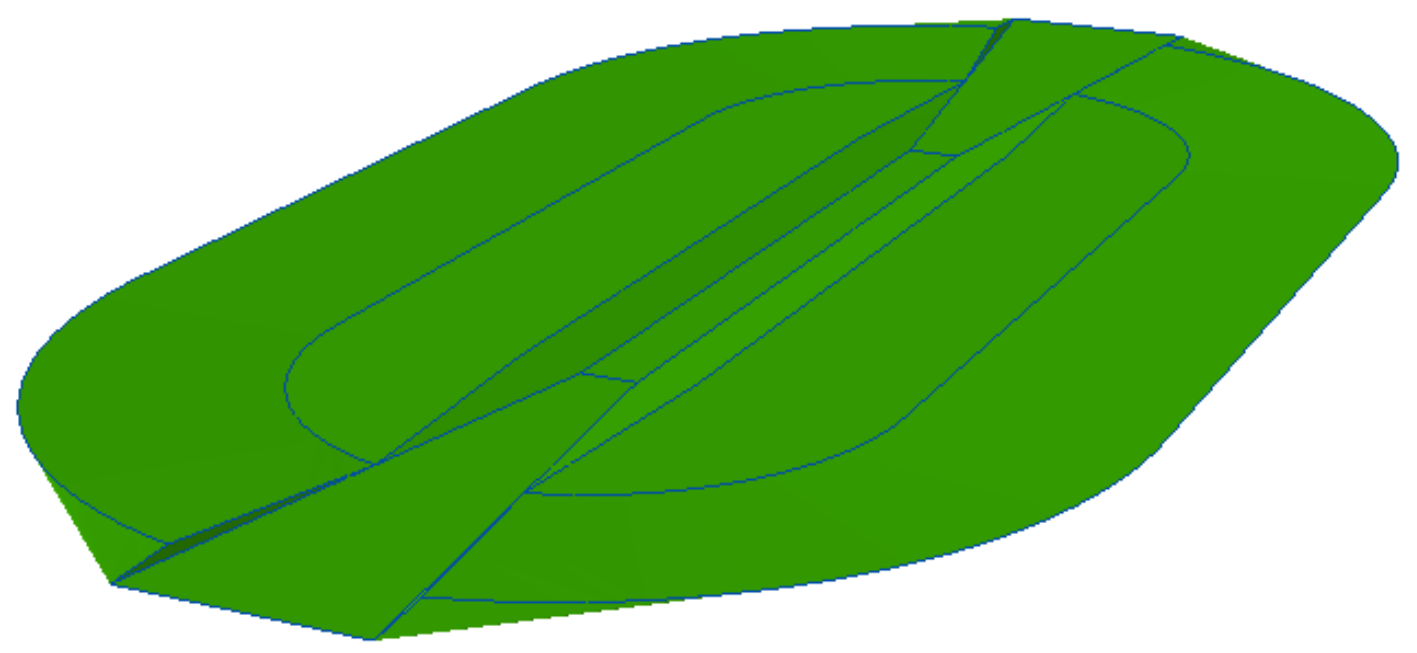

Figure 31: FAA TIN Surface 


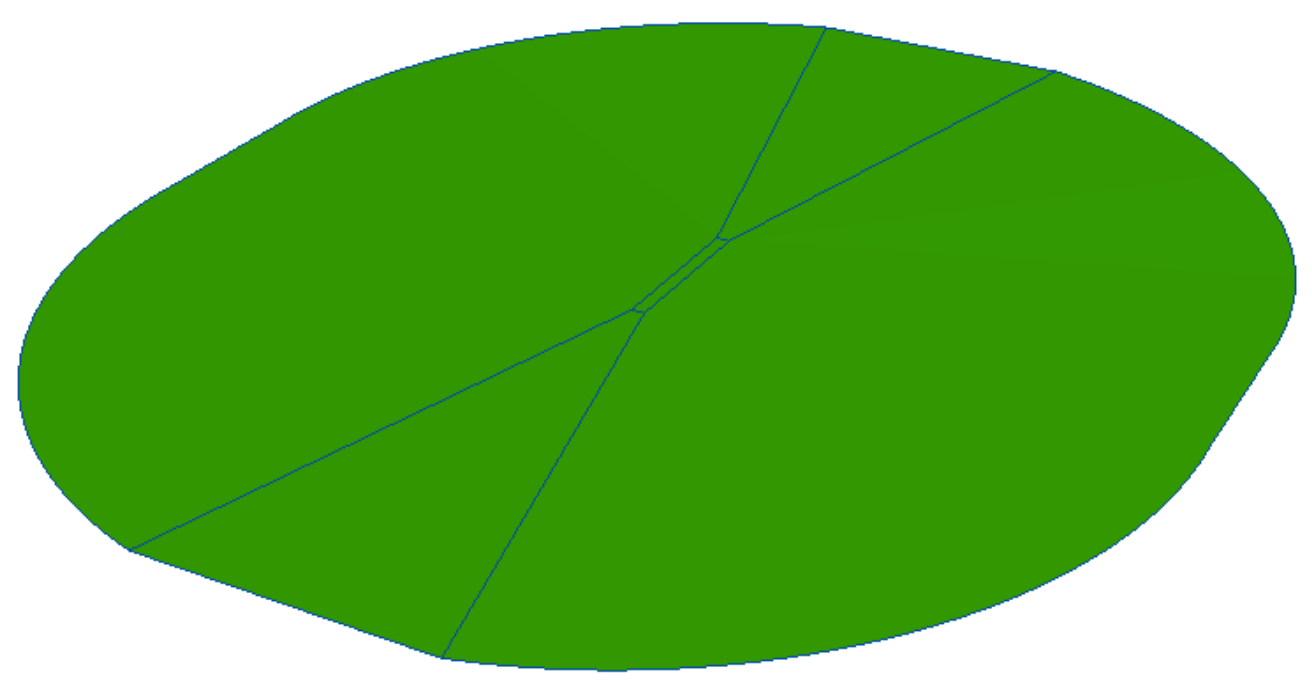

Figure 32: eTOD TIN Surface

In order to yield a better representation of the OIS TIN surfaces in ArcScene, there is a method to exaggerate the slope to produce a better visualization. To do this, the user simply needs to bring up the layer property and locate the "Base Height" tab page. There is a group box called "Elevation from features" and an option called "Use elevation values in the layer features." When this radio button is clicked, the unit and the value can be adjusted. (Kennedy H. , 2004, pp. 18 - 19) For instance, in Figure 33 and Figure 34, both OIS TIN surfaces have been exaggerated by a factor of 10 in custom units and the representation of the elevation is much better than in Figure 31 and Figure 32. 


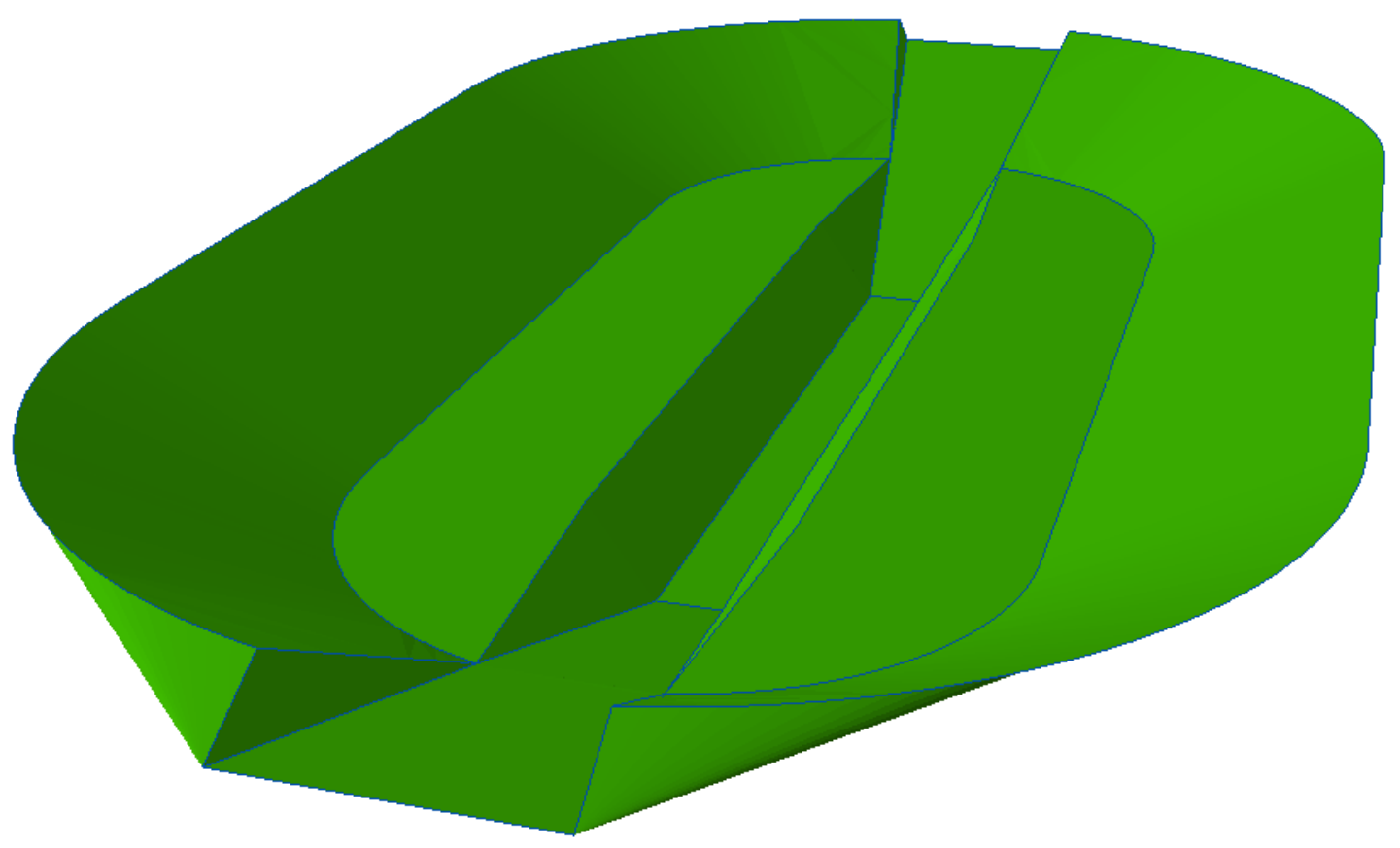

Figure 33: FAA TIN Surface Exaggerated by Factor of 10

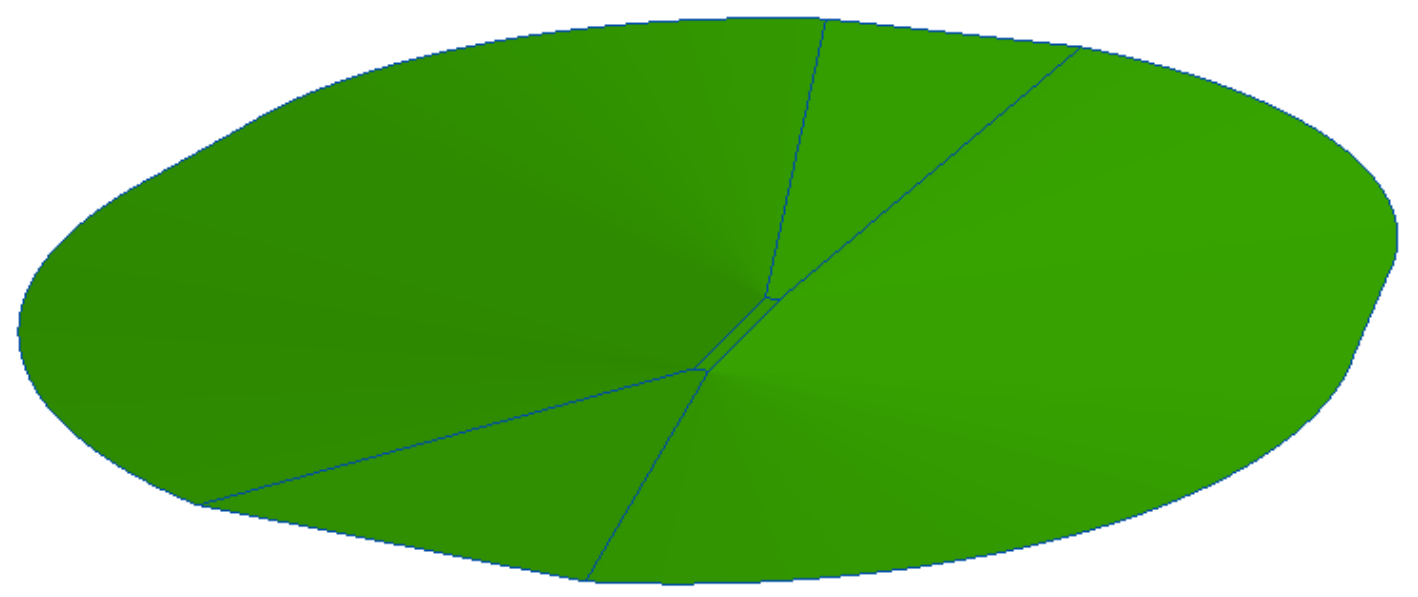

Figure 34: eTOD TIN Surface Exaggerated by Factor 10 
Although the OIS TIN surfaces offer a better representation after exaggerating the slope, these surfaces still look like polygons filled with color. In order to better understand how the OIS TIN Surfaces are formatted, node and edge symbols can be used to aid in this analysis. (Kennedy H. , 2004, p. 174) To replace the current symbols, the user first opens the layer property dialog and locates the "Symbology" tab page, then clicks the "Add" button to add the node and edge symbols in the symbol list. After that, the user unchecks the "Edge" and "Edge Type" and the OIS TIN surfaces will display in nodes and edges. Figure 35 and Figure 37 show the FAA and eTOD TIN surfaces with node and edge symbols. The red points specify the nodes and the green lines and the blues line indicate the edges. Figure 36 and Figure 38 display the zoomed TIN surfaces with node and edge symbols. Characteristics that can be discerned from these two figures are the density of the nodes and that the edges are highly concentrated at the arc. This phenomenon is due to many nodes being added along the arc to make it smoother and the programmatic method to add nodes along the arc with uniform length is called "Densify". For the FAA OIS TIN surfaces, it was constructed using 4474 triangles and 2724 nodes. For eTOD OIS TIN surfaces, it was constructed with 5514 triangles and 5366 nodes. 


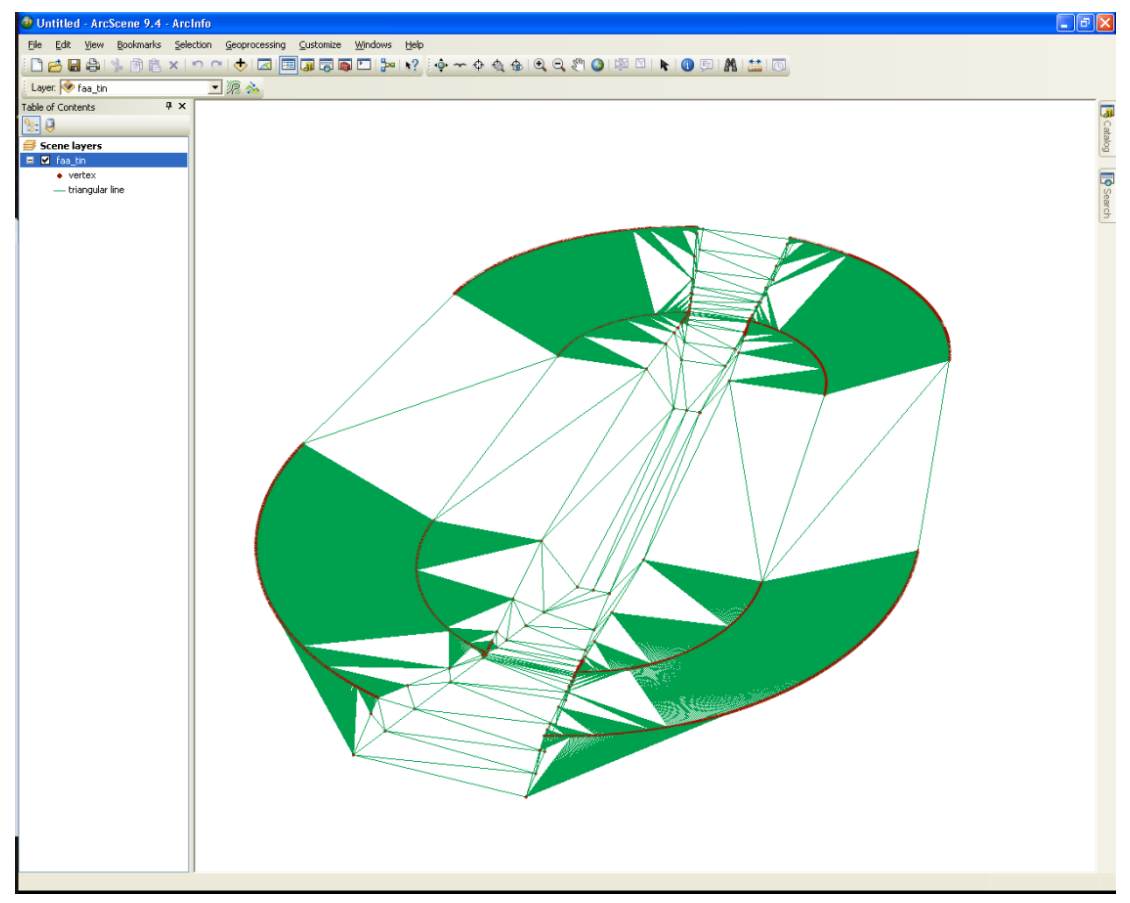

Figure 35: FAA TIN Surface with Node and Edges Symbols

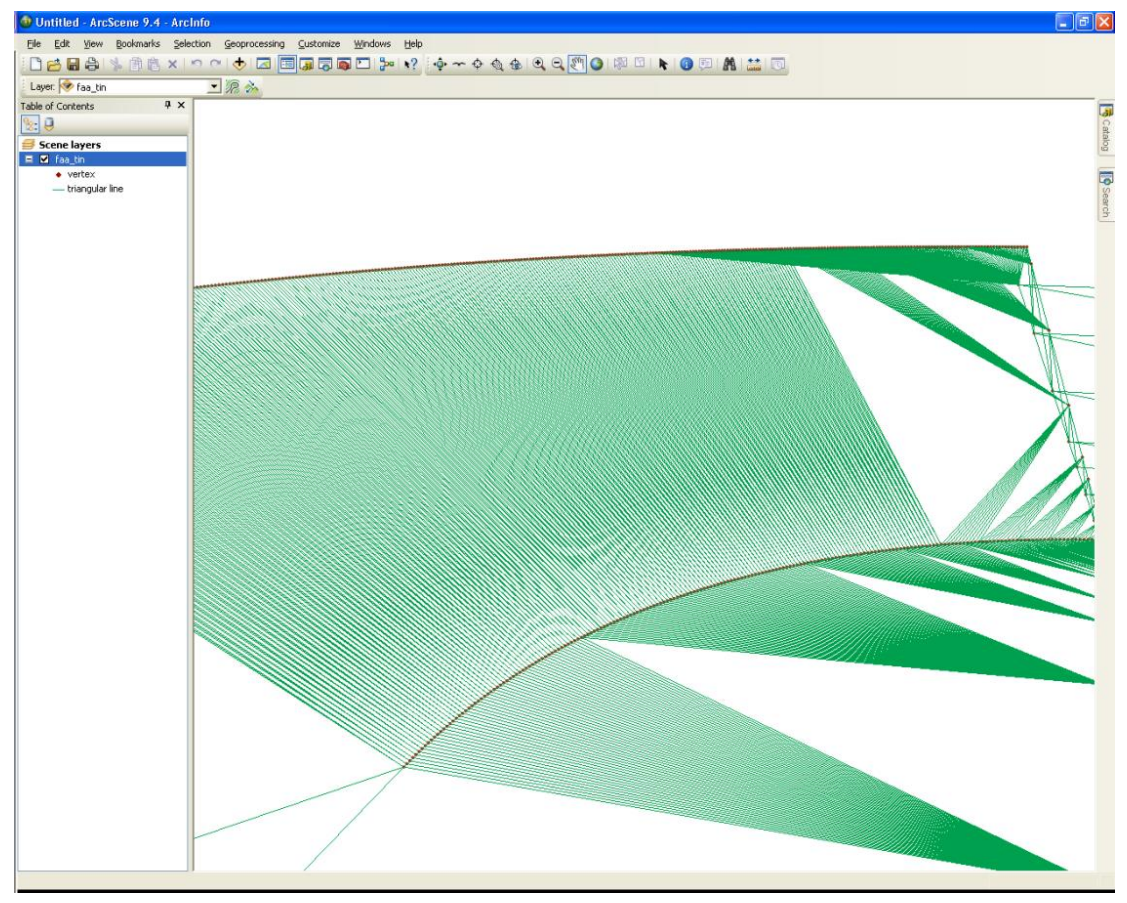

Figure 36: Zoomed FAA TIN Surface with Node and Edge Symbols 


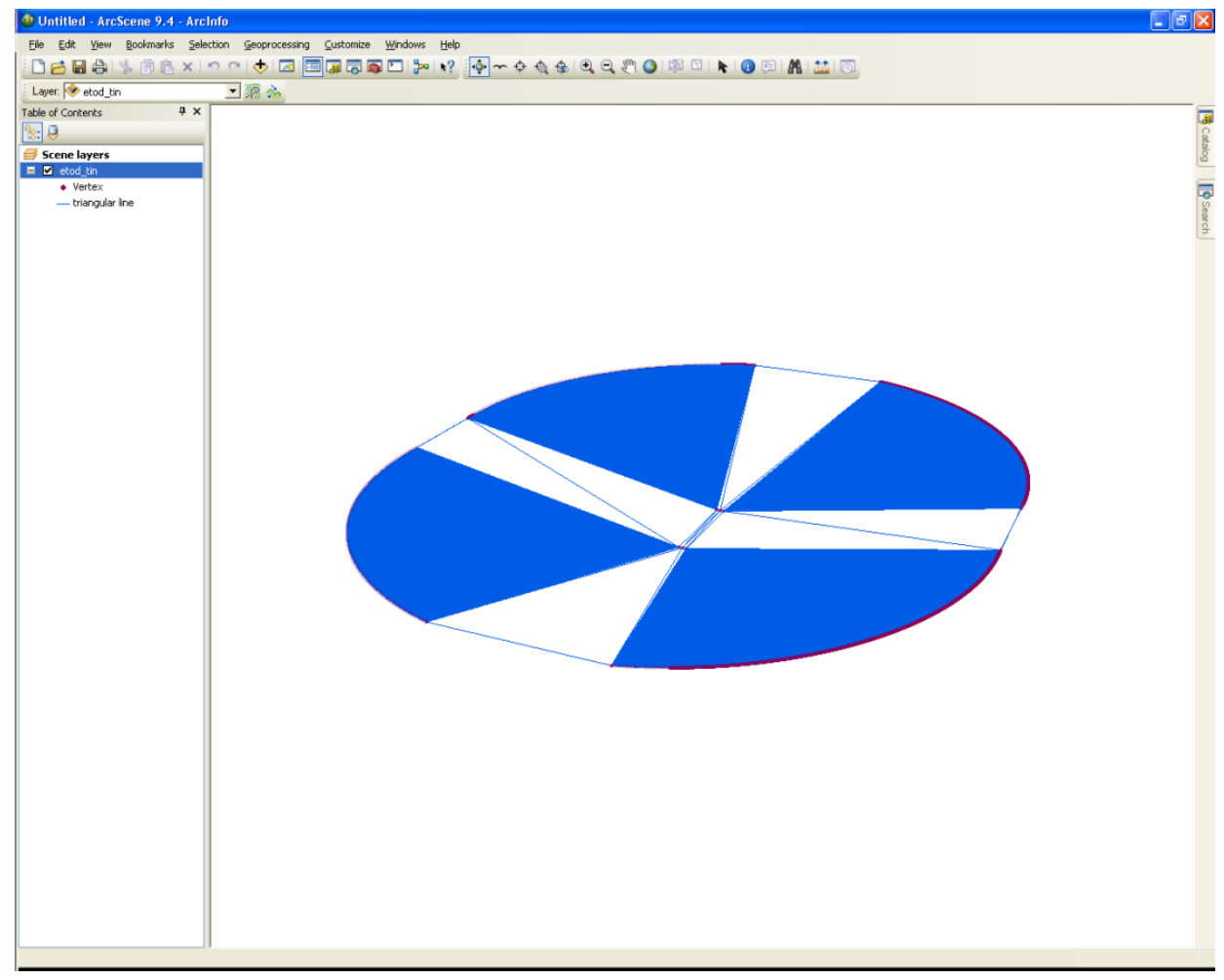

Figure 37: eTOD TIN Surface with Node and Edge Symbols

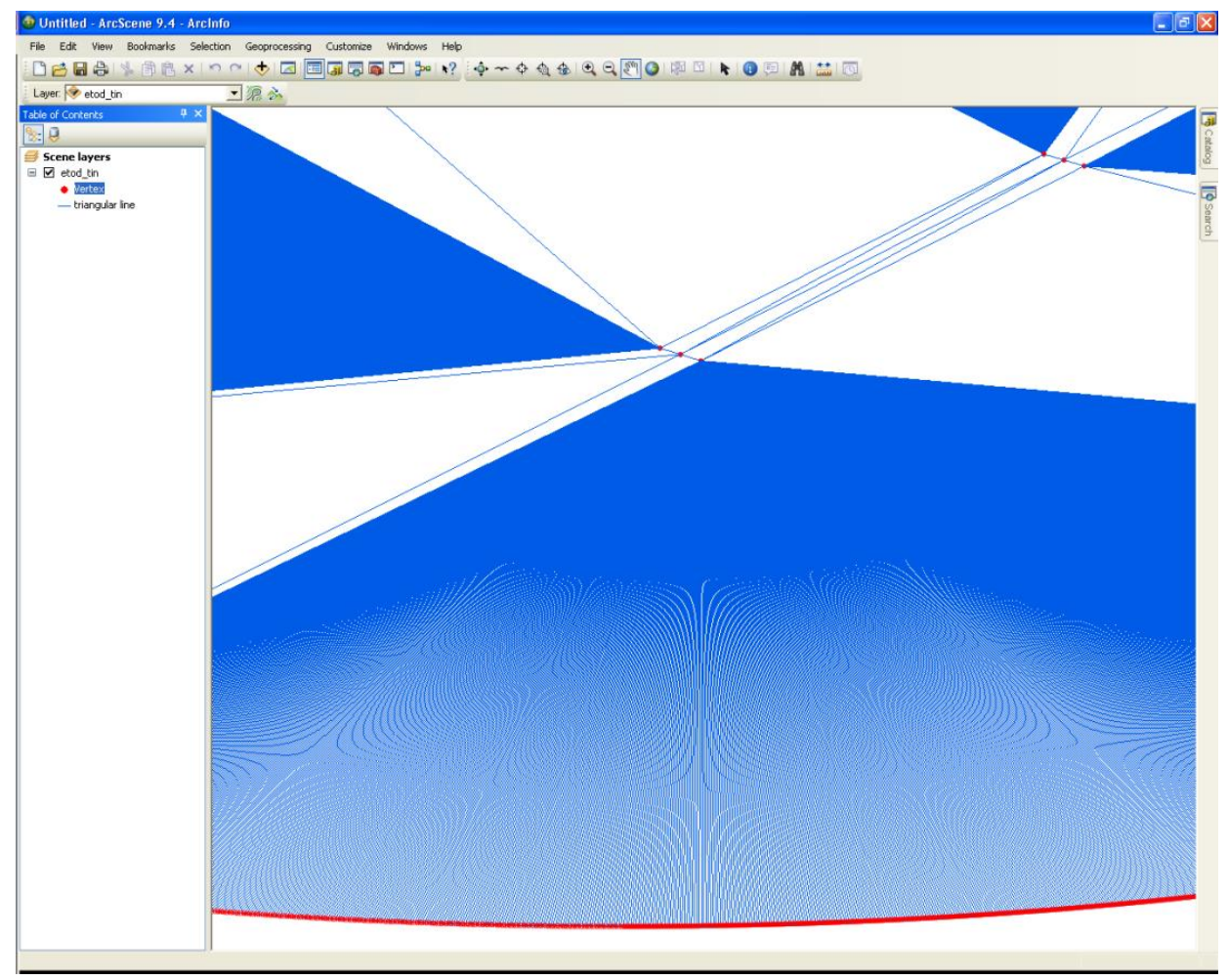

Figure 38: Zoomed eTON TIN Surface with Node and Edge Symbols 
In addition to the node and edge symbols, aspect symbols can be chosen from the symbology list to aid in understanding the direction of the TIN surface. (Kennedy H. , 2004, p. 175) When the aspect symbol replaces the node and edge symbols, the FAA and the eTOD OIS TIN surfaces change to several colored areas. In Figures 39 and 40, the aspect symbol in the table of contents classifies the OIS into nine categories. Red represents that the surface is facing north, orange represents that the surface is facing north-east, yellow represents that the surface is facing east, green represents that the surface is facing south-east, cyan represents that the surface is facing south, light-blue represents that the surface is facing south-west, blue represents that the surface is facing west, pink represents that the surface is facing north-west, and gray represents that the surface is flat.

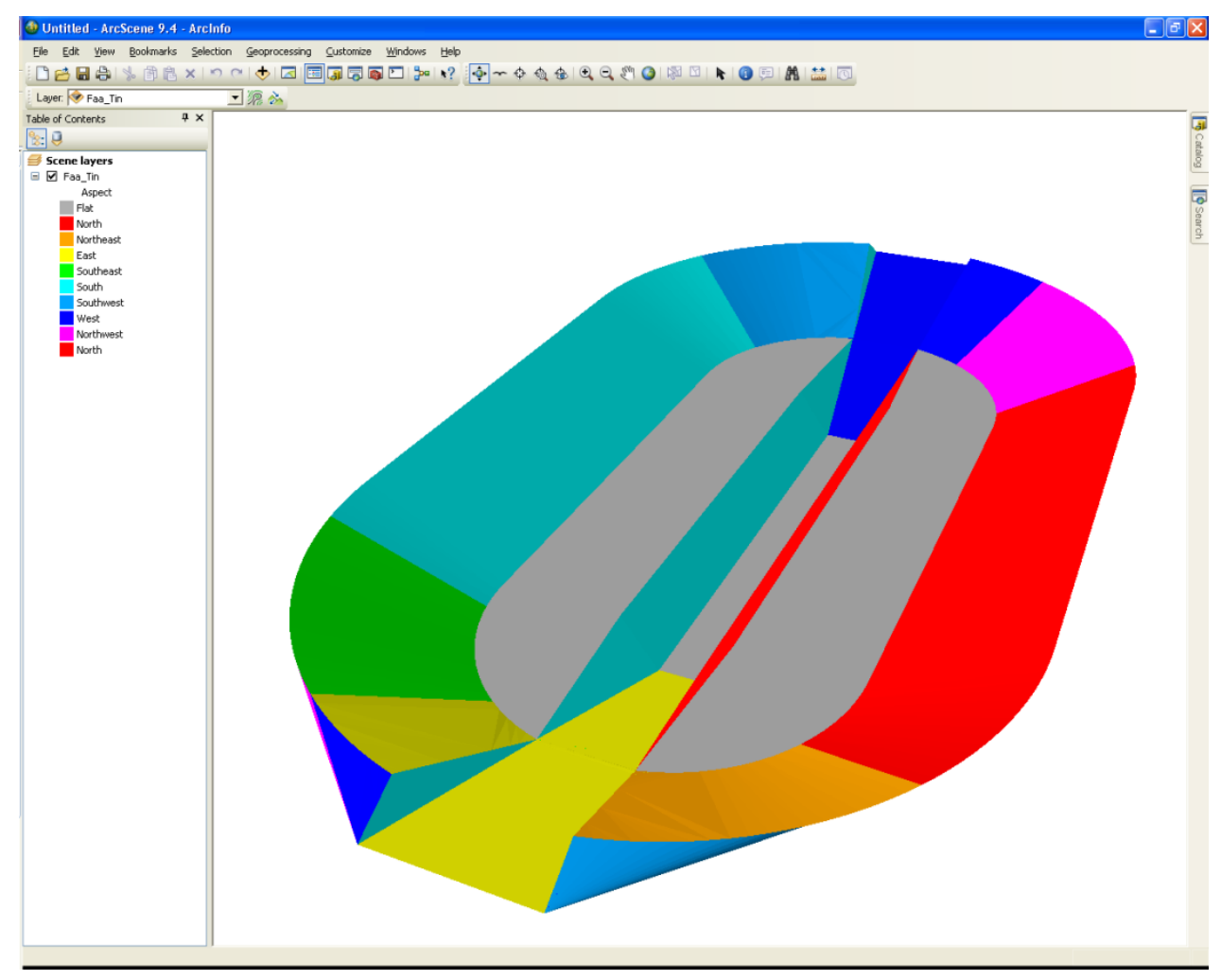

Figure 39: FAA OIS TIN Surface with Aspect Symbol 


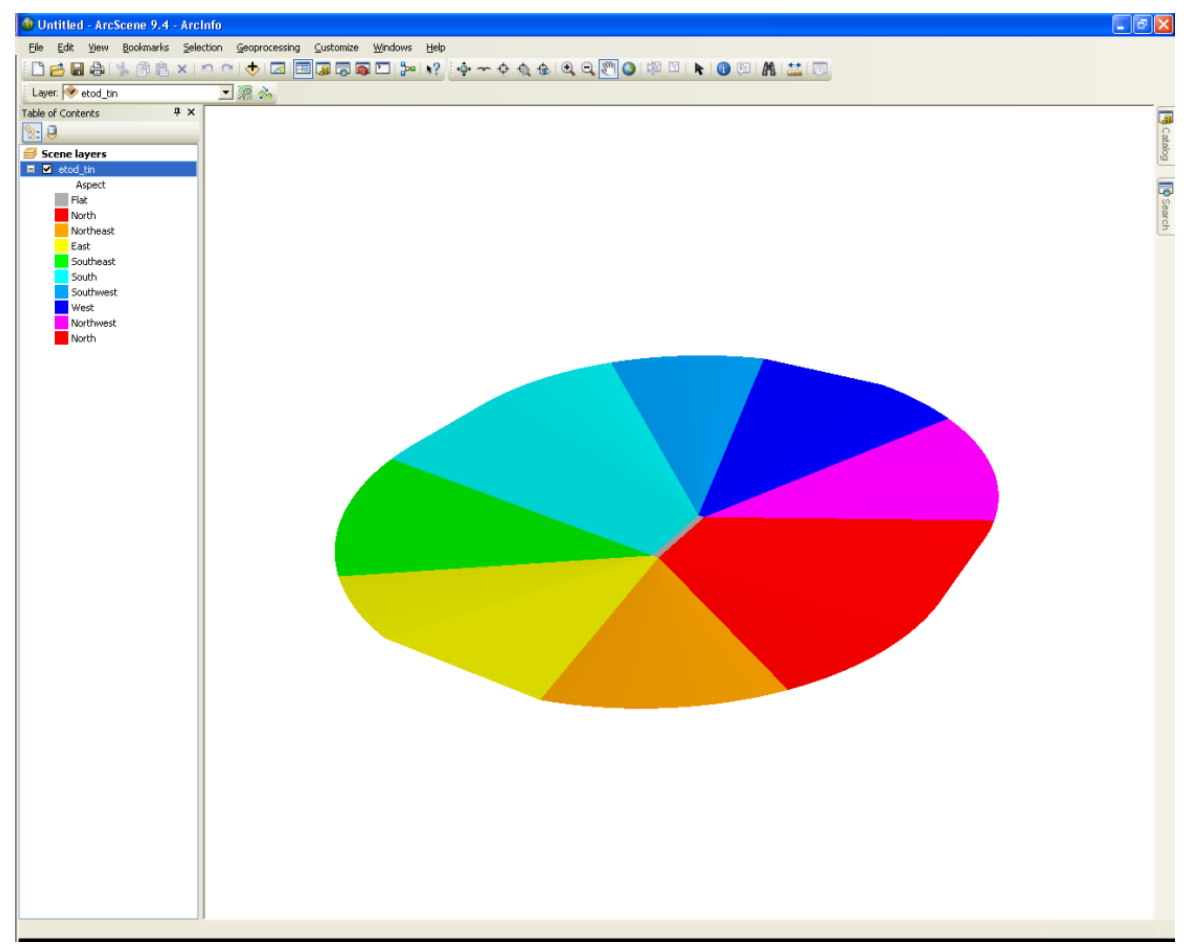

Figure 40: eTOD OIS TIN Surface with Aspect Symbol

The last symbol, elevation representation of the OIS, is of greater relative importance in this section. (Kennedy H. , 2004, p. 178) It is especially useful for future development in areas such as obstacle penetration analysis. In Figures 41 and 42, the elevation symbols in the table of contents categorize the elevation into nine levels. The highest elevation for the FAA OIS is 106.63 meters and for the eTOD OIS is 188.49 meters. 


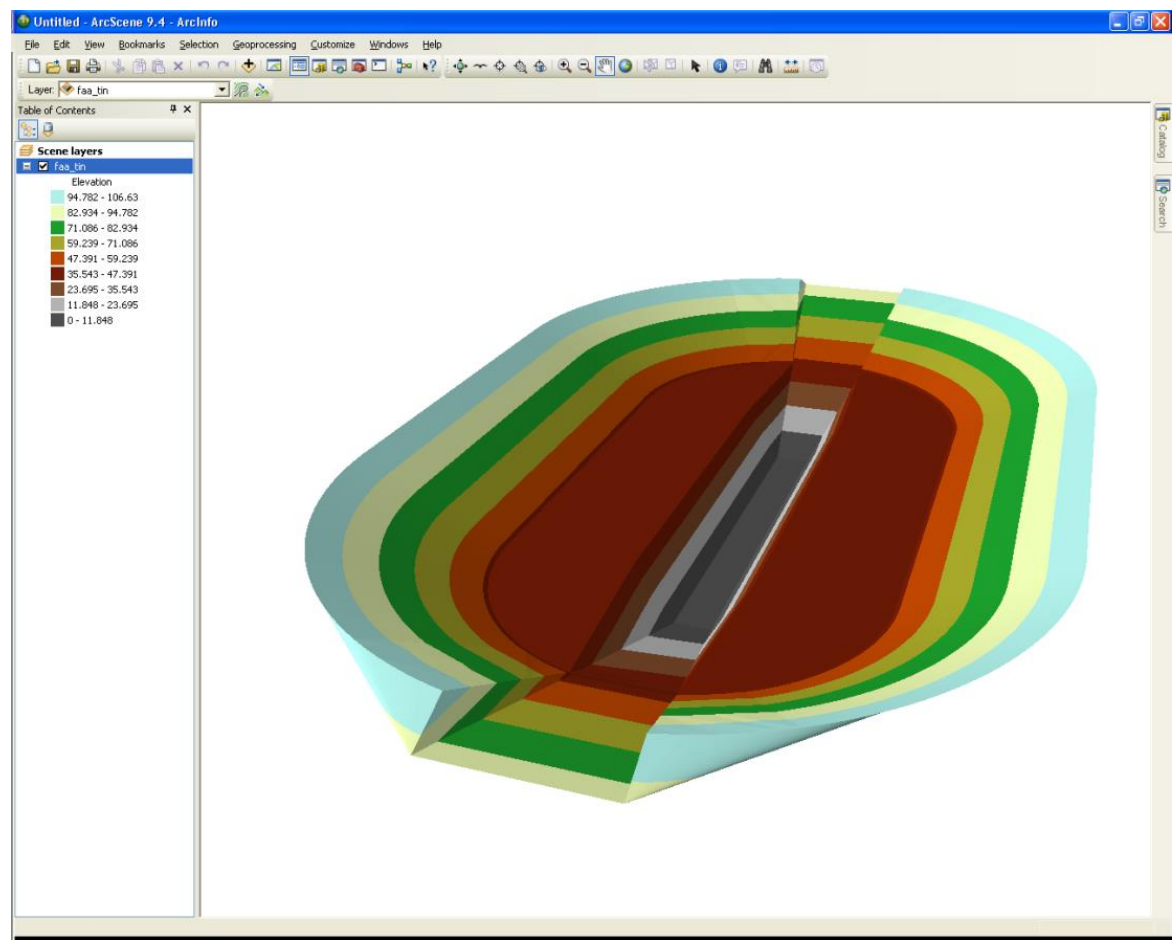

Figure 41： FAA TIN Surface with Elevation Symbol

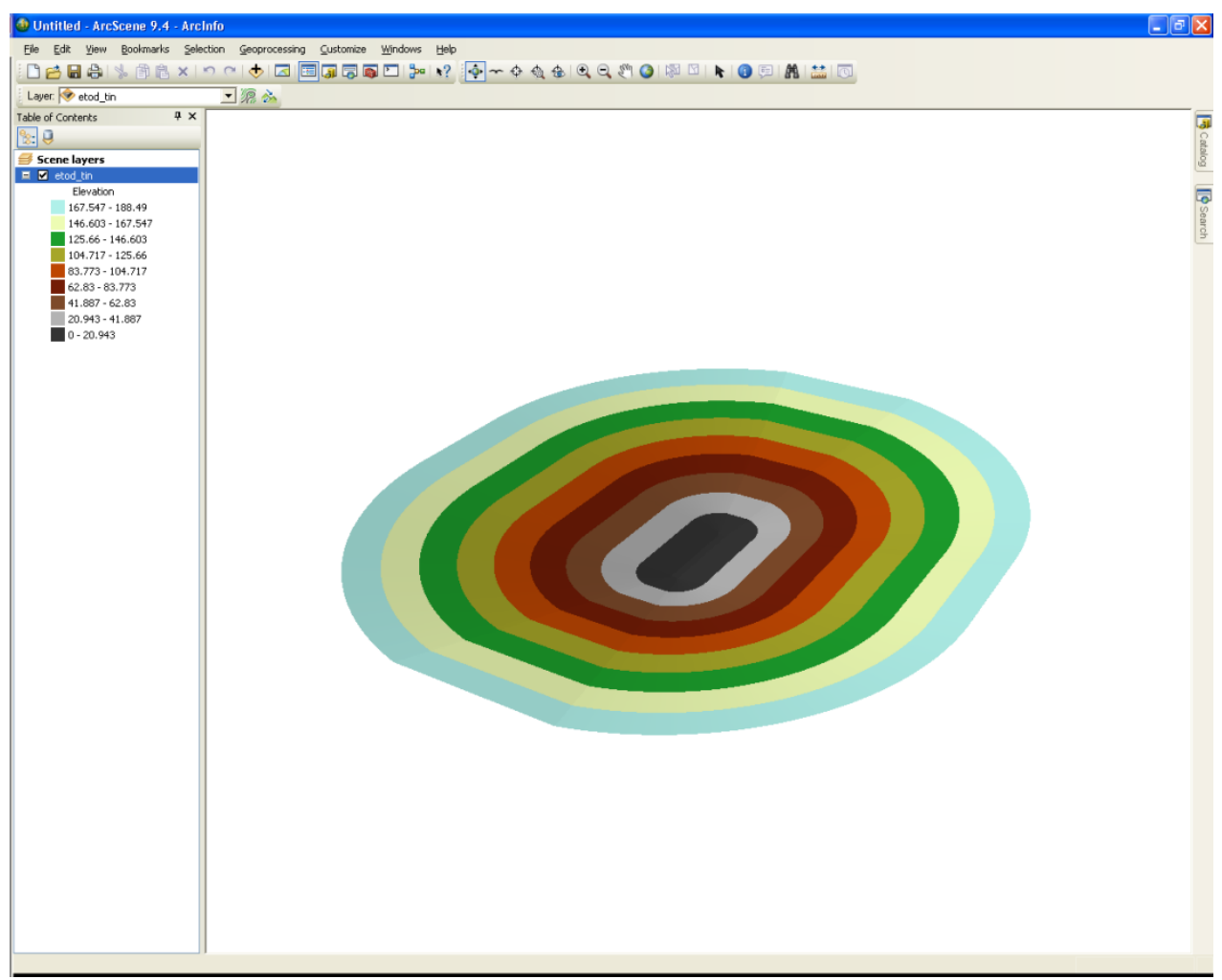

Figure 42: eTOD TIN Surface with Elevation Symbol 



\section{Chapter 8 - Conclusion and Future Work}

This final chapter is composed of section 8.1 and 8.2. Section 8.1 explains why this project was done, what the project did, how its goals were accomplished, and then describes the results. Finally this chapter concludes with a discussion of future work that can be built upon this project.

\subsection{Conclusion}

Air traffic control is an important element in providing a safe air travel environment. Many accidents or near misses involving aircraft occur near landing sites due to aeronautical charts being in different formats, using outdated aeronautical information, or employing different aeronautical language. As a leading company in the field of GIS, ESRI has established PLTS for ArcGIS - Aeronautical Solution to provide a more universal and therefore safer method to model and track flight paths, manage aeronautical information, analyze aeronautical data, and produce complex charts from a central database.

In order to have a safe travel environment, OIS is one of the major elements available to help manage facilities which govern both air and land, to model and monitor noise, to track environmental compliance, to manage construction, and to plan traffic. Because of the significance of OIS, this project aimed to develop a digital method which would increase the accuracy of OIS, improve the effectiveness of its generation, and digitize the OIS data.

There were two major goals in functional requirements which were accomplished in this project. The first one was to design and develop a tool that would be able to generate the OIS features in a more effective way. The second was to store the generated 
OIS feature in a more globalized format. To accomplish the above goals, a GP tool was developed to generate the OIS features. This tool greatly improved the OIS in terms of processing time and maintenance. When there is an OIS update, customers can benefit from this tool by being able to more quickly and accurately generate a new set of OIS and print a new OIS chart without having to start from scratch. In addition, because all the OIS features are stored in the geodatabase, users can easily share the OIS features with other user communities. With the digital OIS data, local governments can enhance city planning by overlaying this data on city infrastructure data.

This project also completed several non-functional requirements. First, it was completed under the ESRI Aeronautical and PLTS development network system and allows the developers from ESRI Aeronautical Solution to plug the OIS GP tool into the Aeronautical Solution. Second, the tool is built upon the current version of ArcGIS libraries and thus will increase its compatibility with the next release. Third, this project followed the PLTS standard for development and documentation. Fourth, the tool testing plan was prepared and the initial testing completed by a developer in the Aeronautical Solution team. The only element yet to be accomplished is the proposed unit test solution in N-Unit application. This could not be completed due to the limitations of the development cycle. Therefore, the unit testing solution has been moved to the future work section.

Overall the project fulfilled the client's requirements and expectations. The result of this project is a significant step toward safer air travel by creating OIS in an effective way in a digital, globalized format. In addition, the OIS GP tool has great potential for 
future development based upon this project. The following section describes these potential future developments.

\subsection{Future Work}

After the client presented the OIS GP tool at the aeronautical user group meeting, significant interest was expressed by other users. Based on discussions with the client after the aeronautical user group meeting, there are several potential enhancements or extensions to this project.

One extended function that can be added into the current OIS GP tool is the intersection for two OIS. Many airports contain more than one runway and it is a major challenge for OIS representation. No matter how the runways intersect or even if they run parallel to each other, some area from one set of OIS should be overlapped to another set of OIS. Hence, many calculations will be involved in this type of OIS generation. Also an in-depth interpretation and thorough explanations are needed in order to create a solid presentation for this OIS model. Another extended function that can be added to the current tool is the one open-ended runway OIS generation. Due to their geographic location, some airports can only have one open-ended runway which allows airplanes to approach and depart from a single direction. With this particular type of runway, the OIS GP tool needs to add an additional parameter for OIS generation because the direction of the runway is involved in the OIS generation. In addition to these extended functions, a further way to extend the current tool is to combine it with another GP tool by using Model Builder from the core ArcGIS to perform more complex processes such as TIN surface creation. 
The major objective of OIS features is to identify obstacles around an airfield. By overlaying a terrain surface or building features to the OIS features in three-dimensional applications such as ArcScene or ArcGlobe, users can easily locate obstacles around the airfield. Beyond this, more GP tool development can be implemented or combined. For instance, terrain surface or building features which intersect with a TIN surface can be used to generate the obstacle feature layer.

This OIS project is a vital first step towards completing translation of aeronautical data from paper charts to digital format. In addition, with the above enhancements, combined with GIS technologies and concepts, a safer air travel environment can be achieved. 


\section{Works Cited}

Aviation Safety Network. (2008, Febrary 21). Accident description. Retrieved March 16, 2009, from aviation-safety.net: http://aviation-

safety.net/database/record.php?id=20080221-0

Belrose, M. H. (2005, 12). March Air Reserve Base Identification and Characterization of Obstructions to Flight. March Air Reserve Base Identification and Characterization of Obstructions to Flight (Unpublished master thesis). Redlands, California, USA: University of Redlands.

BFU Switzerland. (2004). Final Report No. 1793 by the Aircraft Accident Investigation Bureau. Berne: The Aircraft Accident Investigation Bureau (AAIB) of Switzerland.

ESRI. (2006, June 27). ArcGIS Desktop Help 9.3. Retrieved 5 13, 2009, from An overview of commonly used tools: http://webhelp.esri.com/arcgisdesktop/9.3/index.cfm?TopicName=An_overview_ of_commonly_used_tools

ESRI. (2009, March). PLTS ${ }^{\text {TM }}$ for ArcGIS $®$ —Aeronautical Solution: Implementing eTOD in the AIS Data model. Redlands, CA, US: ESRI.

ESRI. (n.d.). ArcGIS Resources Center Geoprocessing. Retrieved 5 13, 2009, from Geoprocessing Object Model version 9.3: http://resources.esri.com/help/9.3/geoprocessing/pdf/GeoprocessingObjectMode19 3.pdf

ESRI. (n.d.). Building a Custom Geoprocessing Function Tool. Retrieved 5 15, 2009, from ArcGIS Resource Centers ArcGIS Engine using .NET: 
http://resources.esri.com/help/9.3/ArcGISEngine/dotnet/concepts_start.htm\#e7d0 6ae9-a6d1-4248-a7a3-9d5f375f088c.htm

ESRI, Geoprocessing Data Types of Parameters and Environments. (n.d.). Geoprocessing Data Types of Parameters and Environments. Retrieved 5 13, 2009, from ArcGIS Resource Center Geoprocessing: http://webhelp.esri.com/arcgisdesktop/9.3/pdf/geoprocessing_data_types.pdf EUROCONTROL. (2009, January). Draft Proposal For The Provision of Data for Area 2.

FAA. (1993, March). Part 77, Objections Affecting Navigable Airspace. Federal Aviation Regulations.

Harris, R. L., \& Johnson, A. R. (n.d.). Detecting Airfield Vertical Obstructions using Digital Photogrammetry and GIS. Retrieved 5 10, 2009, from BAE Systems: http://www.socetset.com/docs/education/professional_papersBAK/detecting_aeronautical_obstructions.pdf Hughes, D. (1996, April 8). USAF, NTSB, Croatia Probe 737 Crash. Aviation Week \& Space Technology.

International Civil Aviation Organization. (2004). Annex 15 Aeronautical Information Services Twelfth Edition. International Civil Aviation Organization.

Kennedy, H. (2004). Data in Three Dimensions: A Guide to ArcGIS 3D Analyst. New York: Delmar Learning.

Kennedy, H. K. (2009). Introduction to 3D Data. New Jersey: John Wiley \& Sons.

Los Angeles International Airport. (2009, January). Volume of Air Traffic. Los Angeles, CA, US. 
McGaugh, C. L. (2004, June 25). Aeronautical Transformation: Paper to Digits changing the way the National Geospatial Intelligence Agency creates Aeronautical Products. Retrieved August 2009, from ESRI:

http://proceedings.esri.com/library/userconf/proc04/docs/pap2215.pdf

Vidal, Susan; Graham, Douglas; Steele, Jeffrey;. (n.d.). FAA Airport Surveying - GIS

Program. Retrieved 8 29, 2009, from National Oceanic and Atmospheric Administration: http://www.ngs.noaa.gov/AERO/eAOC/p1635.pdf 



\section{Appendix A. OIS Geoprocessing Tool Source Code}

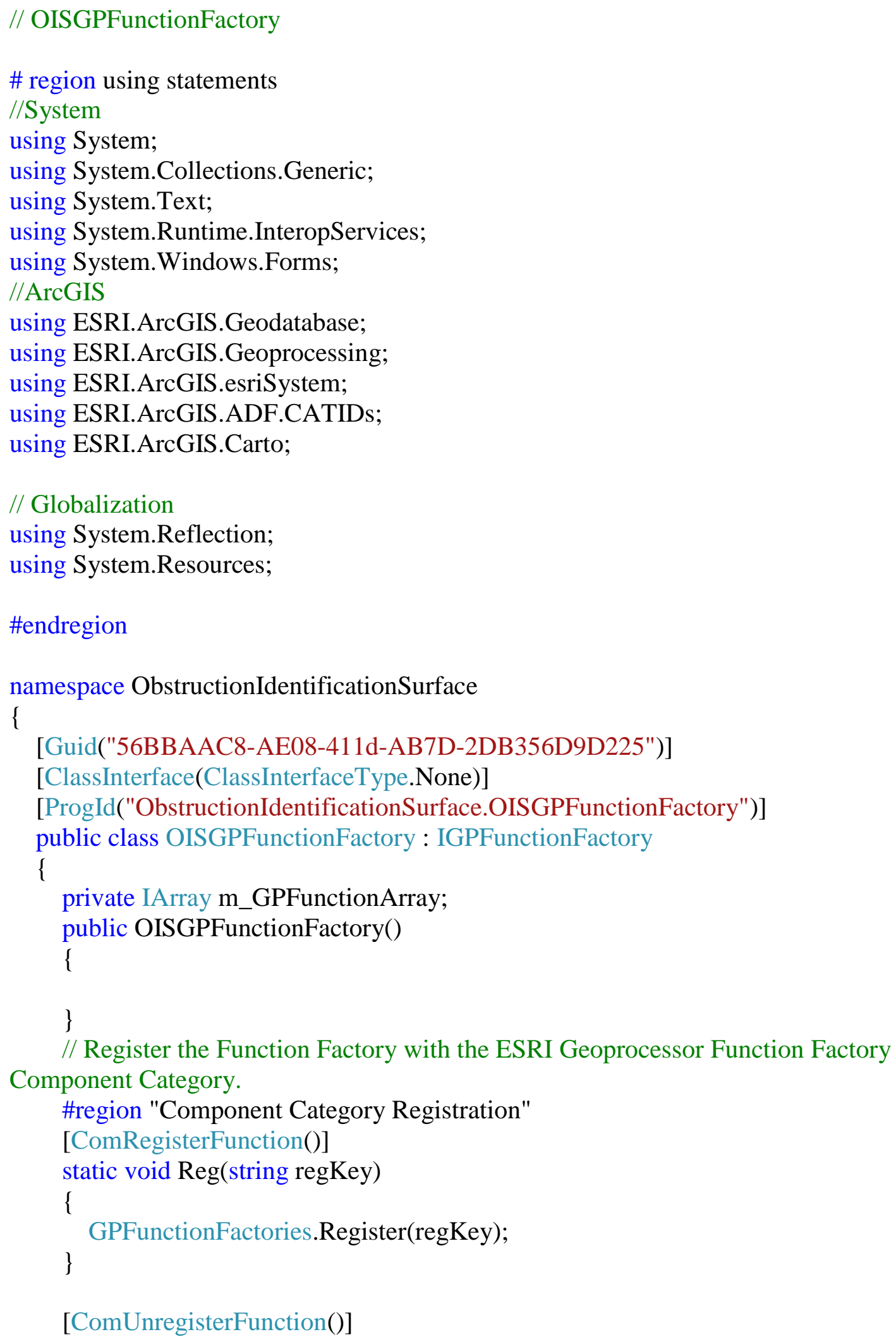




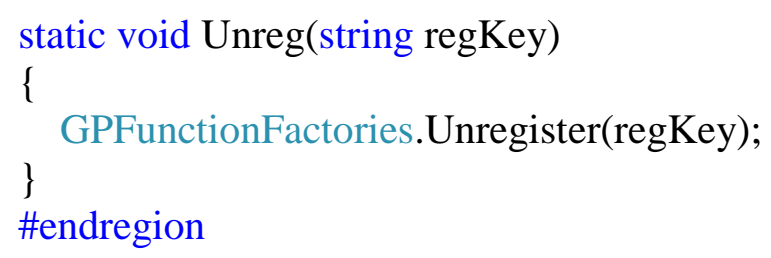


// This is the alias name of the factory.

public string Alias

\{

\}

$$
\text { get }\{\text { return "Obstruction Identification Surfaces"; \} }
$$

// This is the class id of the factory.

public UID CLSID

\{

get

\{

UID id = new UIDClass();

id. Value $=$ this.GetType().GUID.ToString("B");

return id;

\}

\}

// This method will create and return a function object based upon the input name. public IGPFunction GetFunction(string Name)

\{

try

\{

switch (Name)

\{ case ("OIS"):

IGPFunction gpFunction = new OISGP(); return gpFunction;

\}

\}

catch (Exception ex)

\{

MessageBox.Show(ex.Message);

\}

return null;

\}

// This method will create and return a function name object based upon the input name.

public IGPName GetFunctionName(string Name)

\{

try

\{

IGPName gpName = new GPFunctionNameClass();

switch (Name)

\{ 


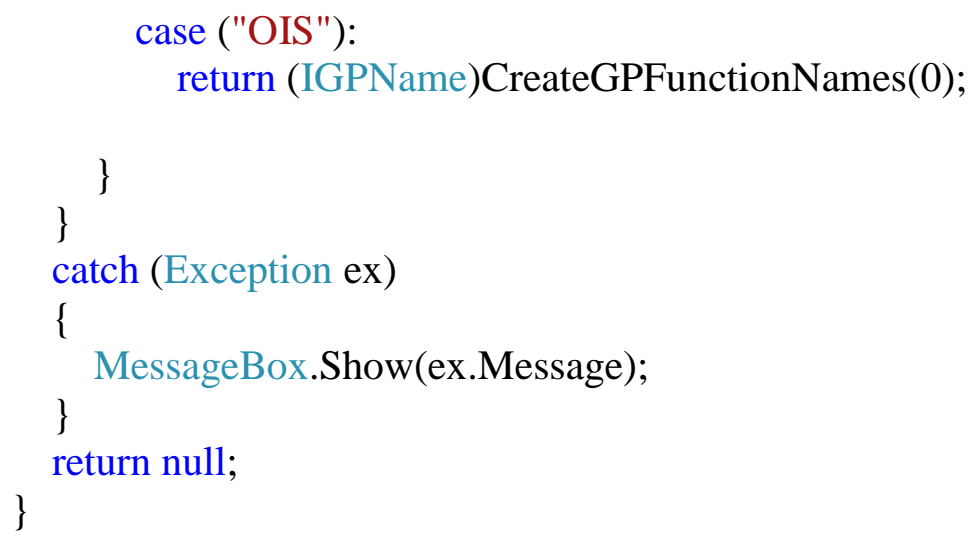

// This method will create and return an enumeration of GPEnvironment objects. // If tools published by this function factory required new environment settings, //then you would define the additional environment settings here.

// This would be similar to how parameters are defined. public IEnumGPEnvironment GetFunctionEnvironments() 


\section{// OISInputParameters}

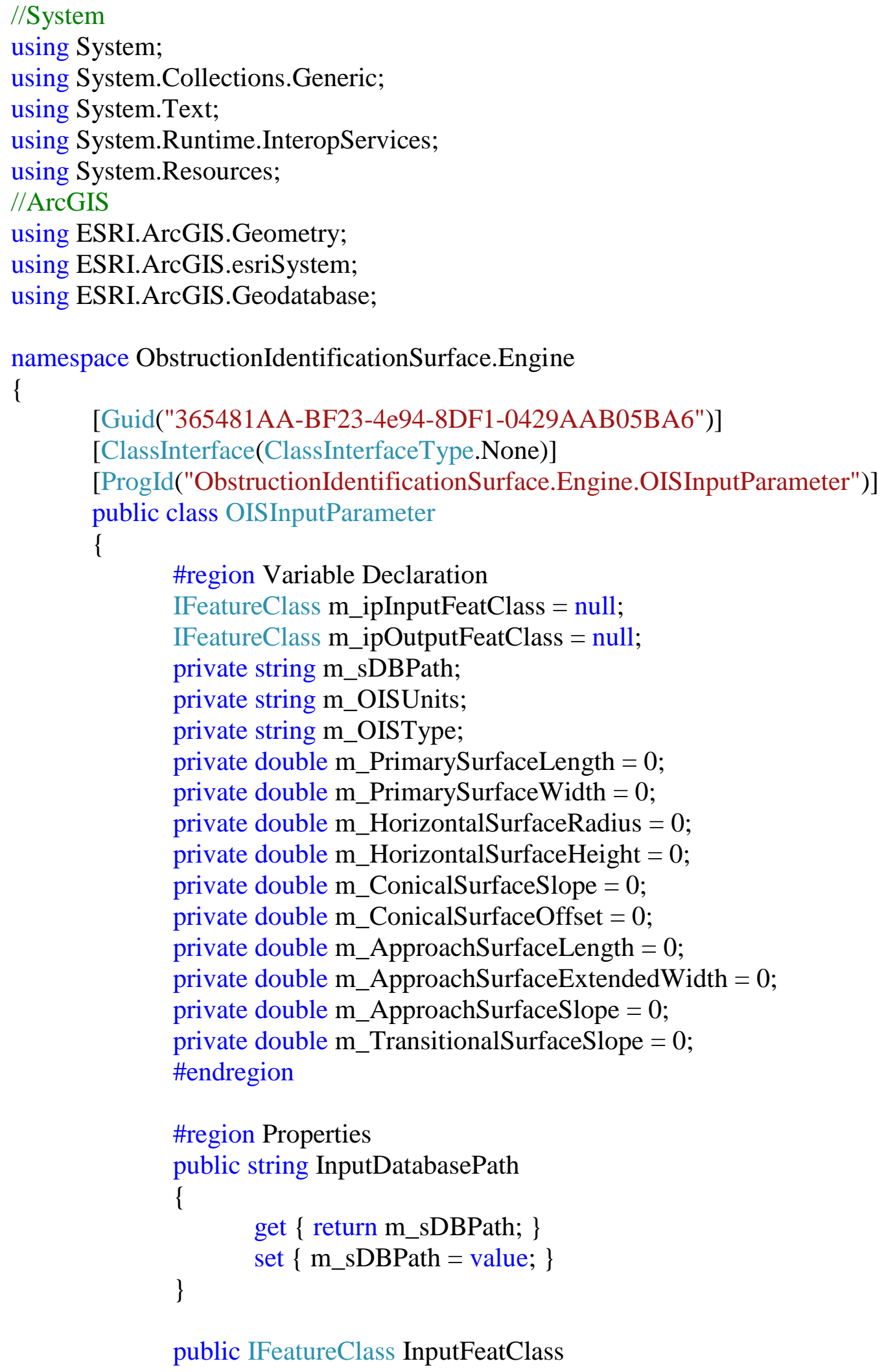




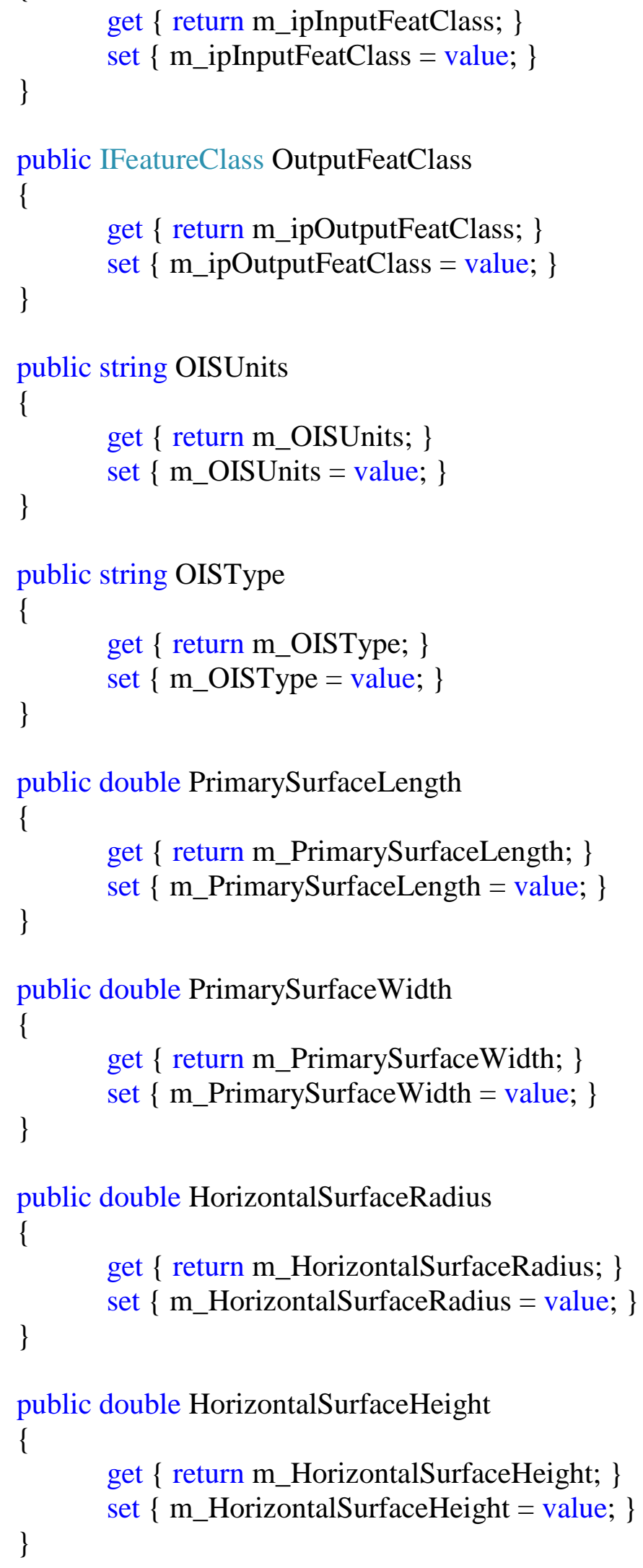


public double ConicalSurfaceOffset

\{ get $\{$ return m_ConicalSurfaceOffset; $\}$ 
using System.Resources;

using ESRI.ArcGIS.esriSystem;

using ESRI.ArcGIS.Geometry;

using ESRI.ArcGIS.Geodatabase;

using ESRI.ArcGIS.Geoprocessing;

using System.Windows.Forms;

using ObstructionIdentificationSurface.Engine;

namespace ObstructionIdentificationSurface

\{

[Guid("71CB68F9-A1D3-4f02-B158-979E0F16D9BF")]

[ClassInterface(ClassInterfaceType.None)]

[ProgId("ObstructionIdentificationSurface.OISGP")]

public class OISGP : IGPFunction2

\{

// Local members

private string $\mathrm{m}_{-}$ToolName = "OIS"; $\quad$ // GP Function Name

private string $\mathrm{m} \_$metadatafile $=$" ";

private IGPUtilities m_iGPUtilities; // GP Utilities object

private IDEUtilities $\mathrm{m} \_$ipDeUtilites $=$new DEUtilitiesClass();

private IWorkspace m_ipWorkspace;

private IFeatureWorkspace $m \_i p F e a t u r e W o r k s p a c e ;$

private ITable m_ipOISSpecTable;

public string $\mathrm{m} \_$strDBType $=$null;

\#region Constructor

public OISGP()

\{

m_iGPUtilities = new GPUtilitiesClass();

\}

\#endregion

\#region IGPFunction Members

// Set the name of the function tool.

// This name appears when executing the tool at the command line or in scripting.

// This name should be unique to each toolbox and must not contain spaces.

public string Name

\{

\}

get $\left\{\right.$ return $\mathrm{m}_{-}$ToolName; $\}$

// Set the function tool Display Name as seen in ArcToolbox.

public string DisplayName 


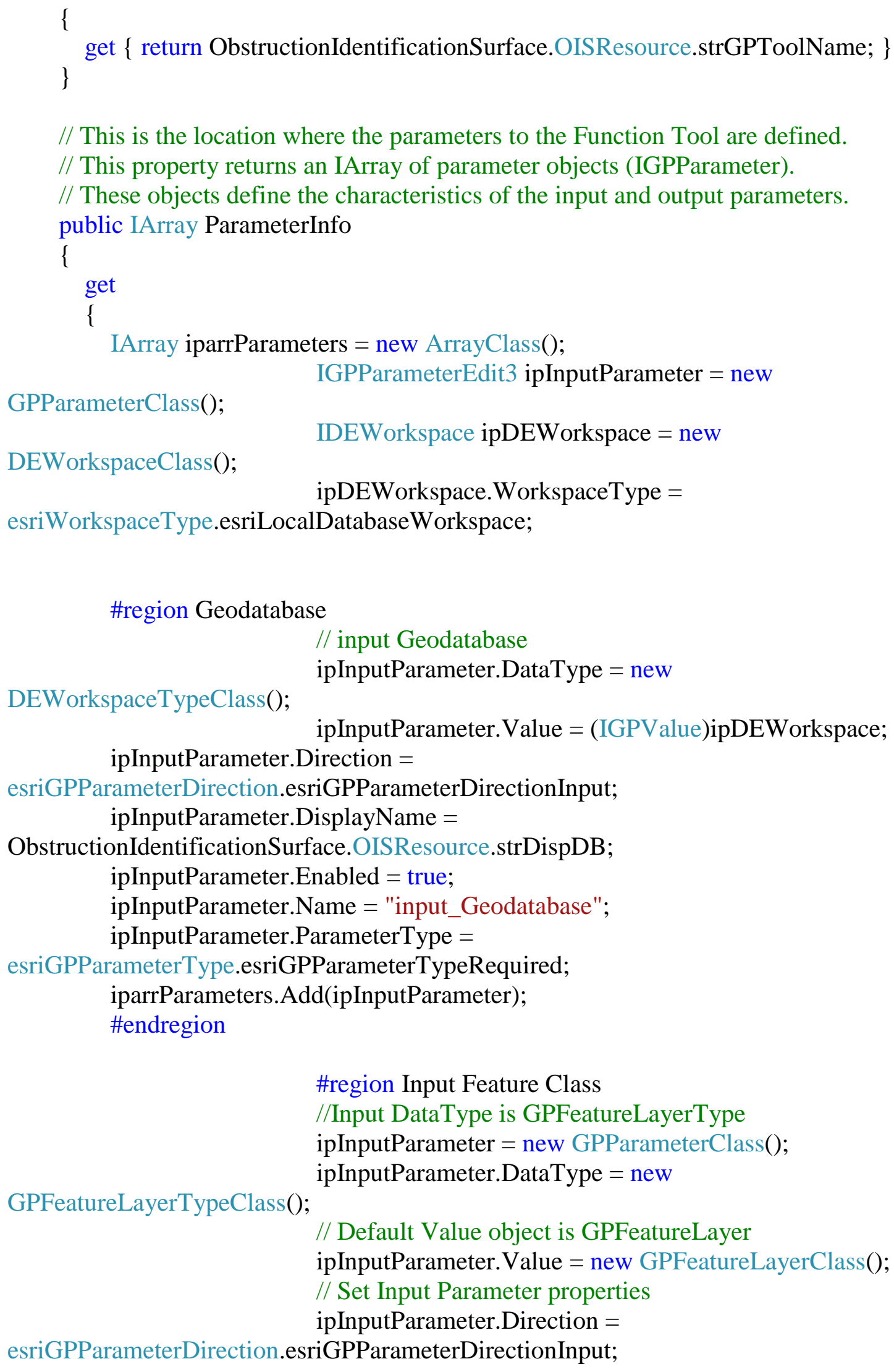




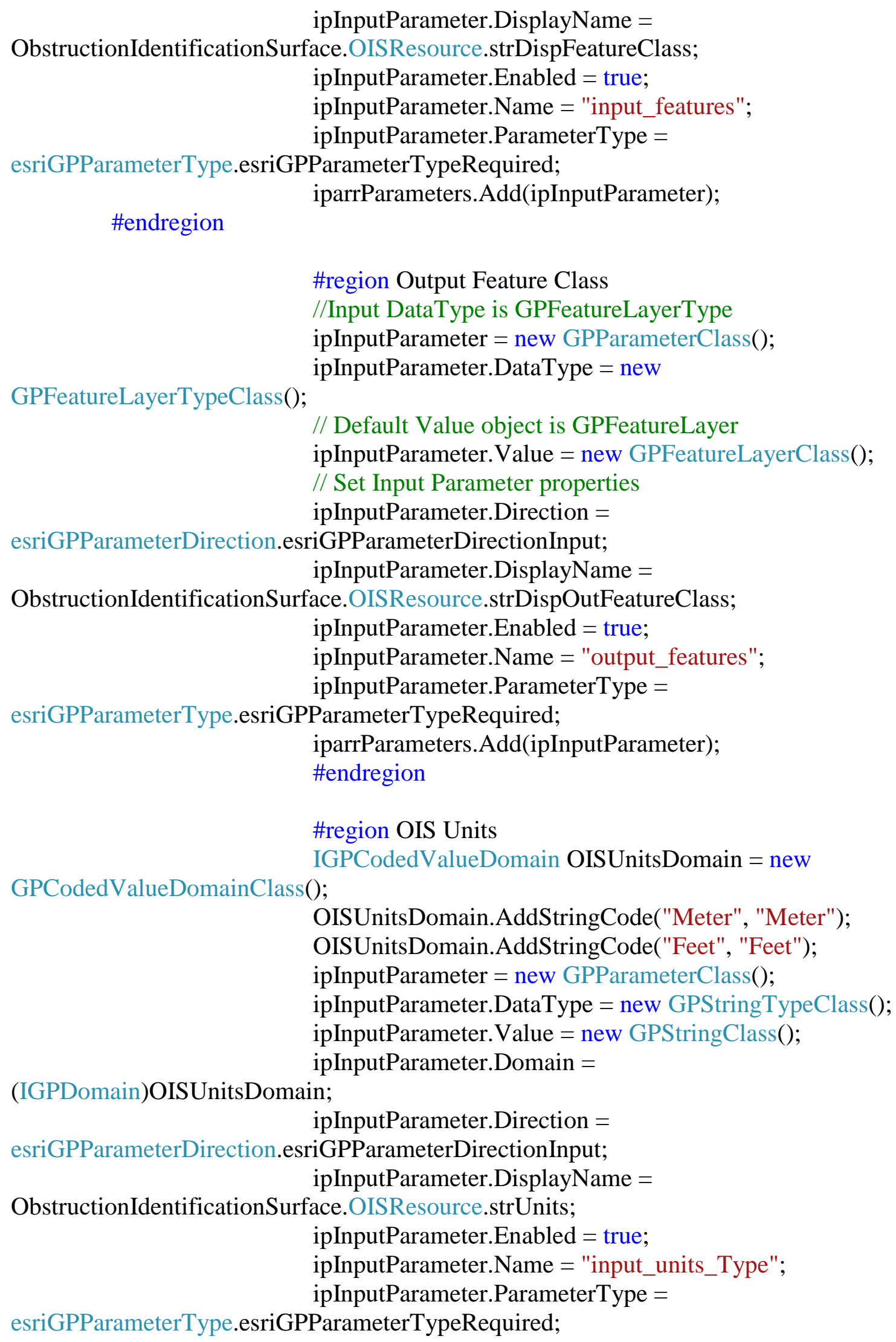


iparrParameters.Add(ipInputParameter);

\#endregion

\#region OIS Type

// input the OIS specification type, either FAA ot eTOD

IGPCodedValueDomain OISspecDomain $=$ new

GPCodedValueDomainClass();

OISspecDomain.AddStringCode("FAA", "FAA");

OISspecDomain.AddStringCode("eTOD", "eTOD");

ipInputParameter = new GPParameterClass();

ipInputParameter.DataType = new GPStringTypeClass();

ipInputParameter.Value = new GPStringClass();

ipInputParameter .Domain $=($ IGPDomain $)$ OISspecDomain;

ipInputParameter.Direction $=$

esriGPParameterDirection.esriGPParameterDirectionInput;

ipInputParameter.DisplayName $=$

ObstructionIdentificationSurface.OISResource.strOISType;

ipInputParameter.Enabled = true;

ipInputParameter.Name = "input_Specification_Type";

ipInputParameter.ParameterType $=$

esriGPParameterType.esriGPParameterTypeRequired;

iparrParameters.Add(ipInputParameter);

\#endregion

\#region Primary Surface

ipInputParameter $=$ new GPParameterClass();

ipInputParameter.DataType = new GPDoubleTypeClass();

ipInputParameter.Value = new GPDoubleClass();

ipInputParameter.Direction $=$

esriGPParameterDirection.esriGPParameterDirectionInput;

ipInputParameter.DisplayName $=$

ObstructionIdentificationSurface.OISResource.strOISPSLength;

ipInputParameter.Enabled = true;

ipInputParameter.Name = "input_ps_length";

ipInputParameter.AddDependency("input_Specification_Type"); ipInputParameter.ParameterType =

esriGPParameterType.esriGPParameterTypeRequired;

iparrParameters.Add(ipInputParameter);

ipInputParameter $=$ new GPParameterClass();

ipInputParameter.DataType $=$ new GPDoubleTypeClass();

ipInputParameter.Value = new GPDoubleClass();

ipInputParameter.Direction $=$

esriGPParameterDirection.esriGPParameterDirectionInput; 
ipInputParameter.DisplayName $=$

ObstructionIdentificationSurface.OISResource.strOISPSWidth;

ipInputParameter.Enabled $=$ true;

ipInputParameter.Name = "input_ps_width";

ipInputParameter.AddDependency("input_Specification_Type");

ipInputParameter.ParameterType $=$

esriGPParameterType.esriGPParameterTypeRequired;

iparrParameters.Add(ipInputParameter);

\#endregion

\#region Horizontal Surface

ipInputParameter $=$ new GPParameterClass();

ipInputParameter.DataType = new GPDoubleTypeClass();

ipInputParameter.Value = new GPDoubleClass();

ipInputParameter.Direction =

esriGPParameterDirection.esriGPParameterDirectionInput; ipInputParameter.DisplayName =

ObstructionIdentificationSurface.OISResource.strOISHSRadius;

ipInputParameter.Enabled = true;

ipInputParameter.Name = "input_hs_radius";

ipInputParameter.AddDependency("input_Specification_Type");

ipInputParameter.ParameterType $=$

esriGPParameterType.esriGPParameterTypeRequired;

iparrParameters.Add(ipInputParameter);

ipInputParameter $=$ new GPParameterClass();

ipInputParameter.DataType = new GPDoubleTypeClass();

ipInputParameter.Value = new GPDoubleClass();

ipInputParameter.Direction $=$

esriGPParameterDirection.esriGPParameterDirectionInput;

ipInputParameter.DisplayName =

ObstructionIdentificationSurface.OISResource.strOISHSHeight;

ipInputParameter.Enabled = true;

ipInputParameter.Name = "input_hs_height";

ipInputParameter.AddDependency("input_Specification_Type");

ipInputParameter.ParameterType $=$

esriGPParameterType.esriGPParameterTypeRequired;

iparrParameters.Add(ipInputParameter);

\#endregion

\#region Conical Surface

ipInputParameter $=$ new GPParameterClass();

ipInputParameter.DataType $=$ new GPDoubleTypeClass(); 
ipInputParameter.Value $=$ new GPDoubleClass();

ipInputParameter.Direction $=$

esriGPParameterDirection.esriGPParameterDirectionInput; ipInputParameter.DisplayName $=$

ObstructionIdentificationSurface.OISResource.strOISCSSlope;

ipInputParameter.Enabled = true;

ipInputParameter.Name = "input_cs_slope";

ipInputParameter.AddDependency("input_Specification_Type");

ipInputParameter.ParameterType $=$

esriGPParameterType.esriGPParameterTypeRequired;

iparrParameters.Add(ipInputParameter);

ipInputParameter $=$ new GPParameterClass();

ipInputParameter.DataType = new GPDoubleTypeClass();

ipInputParameter.Value = new GPDoubleClass();

ipInputParameter.Direction $=$

esriGPParameterDirection.esriGPParameterDirectionInput;

ipInputParameter.DisplayName =

ObstructionIdentificationSurface.OISResource.strOISCSOffset;

ipInputParameter.Enabled = true;

ipInputParameter.Name = "input_cs_offset";

ipInputParameter.AddDependency("input_Specification_Type");

ipInputParameter.ParameterType $=$

esriGPParameterType.esriGPParameterTypeRequired;

iparrParameters.Add(ipInputParameter);

\#endregion

\#region Approach Surface

ipInputParameter $=$ new GPParameterClass();

ipInputParameter.DataType = new GPDoubleTypeClass();

ipInputParameter.Value = new GPDoubleClass();

ipInputParameter. Direction $=$

esriGPParameterDirection.esriGPParameterDirectionInput;

ipInputParameter.DisplayName $=$

ObstructionIdentificationSurface.OISResource.strOISASLength;

ipInputParameter.Enabled = true;

ipInputParameter.Name = "input_as_length";

ipInputParameter.AddDependency("input_Specification_Type");

ipInputParameter.ParameterType =

esriGPParameterType.esriGPParameterTypeRequired;

iparrParameters.Add(ipInputParameter);

ipInputParameter $=$ new GPParameterClass(); 
ipInputParameter.DataType = new GPDoubleTypeClass();

ipInputParameter.Value = new GPDoubleClass();

ipInputParameter.Direction $=$

esriGPParameterDirection.esriGPParameterDirectionInput;

ipInputParameter.DisplayName =

ObstructionIdentificationSurface.OISResource.strOISASSlope;

ipInputParameter.Enabled = true;

ipInputParameter.Name = "input_as_slope";

ipInputParameter.AddDependency("input_Specification_Type");

ipInputParameter.ParameterType $=$

esriGPParameterType.esriGPParameterTypeRequired;

iparrParameters.Add(ipInputParameter);

ipInputParameter $=$ new GPParameterClass();

ipInputParameter.DataType = new GPDoubleTypeClass();

ipInputParameter.Value = new GPDoubleClass();

ipInputParameter.Direction $=$

esriGPParameterDirection.esriGPParameterDirectionInput;

ipInputParameter.DisplayName $=$

ObstructionIdentificationSurface.OISResource.strOISASExtendWidth;

ipInputParameter.Enabled = true;

ipInputParameter.Name = "input_as_extendedwidth";

ipInputParameter.AddDependency("input_Specification_Type");

ipInputParameter.ParameterType $=$

esriGPParameterType.esriGPParameterTypeRequired;

iparrParameters.Add(ipInputParameter);

\#endregion

\#region Transitional Surface

ipInputParameter $=$ new GPParameterClass();

ipInputParameter.DataType = new GPDoubleTypeClass();

ipInputParameter.Value = new GPDoubleClass();

ipInputParameter.Direction $=$

esriGPParameterDirection.esriGPParameterDirectionInput; ipInputParameter.DisplayName =

ObstructionIdentificationSurface.OISResource.strOISTSSlope;

ipInputParameter.Enabled = true;

ipInputParameter.Name = "input_ts_slope";

ipInputParameter.AddDependency("input_Specification_Type");

ipInputParameter.ParameterType $=$

esriGPParameterType.esriGPParameterTypeRequired;

iparrParameters.Add(ipInputParameter);

\#endregion 


$$
\begin{aligned}
& \text { return iparrParameters; } \\
& \quad \text { /* } \\
& \text { Called each time the user changes a parameter in the tool dialog or }
\end{aligned}
$$$$
\text { Command Line. }
$$

This updates the output data of the tool, which extremely useful for building models.

After returning from UpdateParameters(), geoprocessing calls its internal validation routine checkng that a given set of parameter values

are of the appropriate number, DataType, and value.

This method will update the output parameter value with the unique field.

$* /$

public void UpdateParameters(IArray paramvalues,

IGPEnvironmentManager pEnvMgr)

\{

IGPParameter 3 ipGPDatabase $=$ (IGPParameter3)paramvalues.get_Element(0);

IGPValue ipDatabaseParameterValue $=$

m_iGPUtilities.UnpackGPValue(ipGPDatabase);

IDEWorkspace ipDeWorkspace $=$ ipDatabaseParameterValue as

IDEWorkspace;

as IDataElement);

IDataset ipDataset $=\mathrm{m} \_$ipDeUtilites.OpenDataset $(\mathrm{ipDeWorkspace}$

\author{
m_ipWorkspace $=$ ipDataset as IWorkspace; \\ $\mathrm{m} \_$ipFeatureWorkspace $=($IFeatureWorkspace $) \mathrm{m} \_$ipWorkspace; \\ m_ipOISSpecTable $=$ \\ m_ipFeatureWorkspace.OpenTable("OIS_Spec"); \\ // Retrieve the parameter value \\ IGPParameterEdit3 ipSpecParam = \\ (IGPParameterEdit3)paramvalues.get_Element(4); \\ IGPParameterEdit 3 ipPSLength $=$ \\ (IGPParameterEdit3)paramvalues.get_Element(5); \\ IGPParameterEdit3 ipPSWidth $=$ \\ (IGPParameterEdit3)paramvalues.get_Element(6); \\ IGPParameterEdit 3 ipHSRadius $=$ \\ (IGPParameterEdit3)paramvalues.get_Element(7); \\ IGPParameterEdit 3 ipHSHeight $=$ \\ (IGPParameterEdit3)paramvalues.get_Element(8); \\ IGPParameterEdit3 ipCSSlope $=$ \\ (IGPParameterEdit3)paramvalues.get_Element(9);
}


IGPParameterEdit 3 ipCSOffset $=$ (IGPParameterEdit3)paramvalues.get_Element(10);

IGPParameterEdit3 ipASLength = (IGPParameterEdit3)paramvalues.get_Element(11);

IGPParameterEdit 3 ipASSlope $=$ (IGPParameterEdit3)paramvalues.get_Element(12);

IGPParameterEdit3 ipASExtendedWidth =

(IGPParameterEdit3)paramvalues.get_Element(13);

IGPParameterEdit3 ipTSSlope $=$ (IGPParameterEdit3)paramvalues.get_Element(14);

// If we have an unique field value, create a new field based on the unique field name the user entered.

if ((ipSpecParam as IGPParameter3).Value.IsEmpty ()$==$ false $)$ \{ string sSepecType $=(\mathrm{ipSpecParam}$ as

IGPParameter3).Value.GetAsText().ToLower();

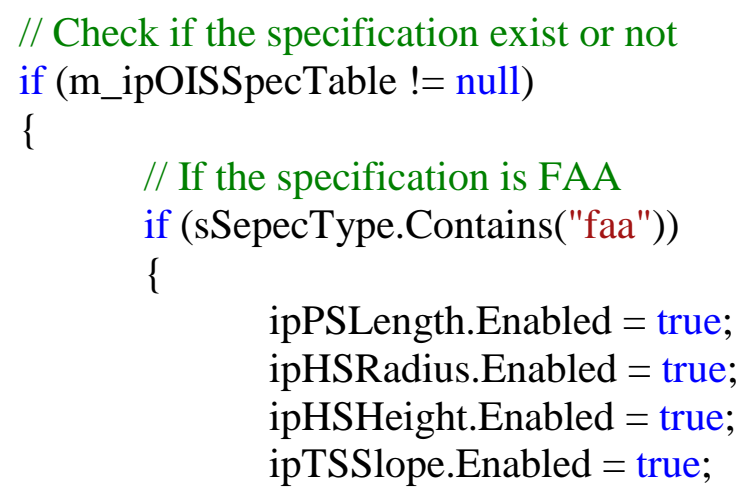

// Check if the specification exist or not if (m_ipOISSpecTable != null)

\{

// If the specification is FAA

if (sSepecType.Contains("faa"))

\{

ipPSLength.Enabled = true;

ipHSRadius.Enabled = true;

ipHSHeight.Enabled = true;

ipTSSlope. Enabled = true;

// Get the cusor from the table and loop

through all the rows and find the right specification

m_ipOISSpecTable.Search(null, false);

ICursor ipCursor $=$

IRow ipRow = ipCursor.NextRow();

while (ipRow != null)

\{

if

(ipRow.get_Value(ipRow.Fields.FindField("Type")).ToString().ToLower().Contains("faa "))

\author{
\{ \\ break; \\ \} \\ \} \\ ipRow $=$ ipCursor.NextRow(); \\ if (ipRow != null)
}


IGPParameter3).Altered == false)

if ((ipPSLength as

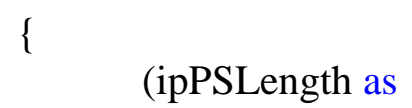

IGPParameter3).Value.SetAsText(ipRow.get_Value(ipRow.Fields.FindField("PS_Length ")).ToString());

\}

if ((ipPSWidth as

IGPParameter3).Altered == false)

(ipPSWidth as

IGPParameter3).Value.SetAsText(ipRow.get_Value(ipRow.Fields.FindField("PS_Width ")).ToString());

IGPParameter3).Altered $==$ false)

\} if ((ipHSRadius as

\{

(ipHSRadius as

IGPParameter3).Value.SetAsText(ipRow.get_Value(ipRow.Fields.FindField("HS_Radiu s")).ToString());

IGPParameter3).Altered == false)

if ((ipHSHeight as

\{

(ipHSHeight as

IGPParameter3).Value.SetAsText(ipRow.get_Value(ipRow.Fields.FindField("HS_Heigh t")).ToString());

IGPParameter3).Altered == false)

\} if ((ipCSSlope as

\{

(ipCSSlope as

IGPParameter3).Value.SetAsText(ipRow.get_Value(ipRow.Fields.FindField("CS_Slope" )).ToString());

IGPParameter3).Altered $==$ false)

\}

if ((ipCSOffset as

IGPP

(ipCSOffset as

IGPParameter3).Value.SetAsText(ipRow.get_Value(ipRow.Fields.FindField("CS_Offset ")).ToString());

IGPParameter3).Altered == false)

\} if ((ipASLength as 
(ipASLength as

IGPParameter3).Value.SetAsText(ipRow.get_Value(ipRow.Fields.FindField("AS_Lengt h")).ToString());

IGPParameter3).Altered == false)

\}

if ((ipASSlope as

\{

IGPParameter3).Value.SetAsText(ipRow.get_Value(ipRow.Fields.FindField("AS_Slope ")).ToString());

IGPParameter3).Altered $==$ false)

\}

if ((ipASExtendedWidth as

\{ (ipASExtendedWidth as

IGPParameter3).Value.SetAsText(ipRow.get_Value(ipRow.Fields.FindField("AS_Exten dedWidth")).ToString());

IGPParameter3).Altered $==$ false)

\}

if ((ipTSSlope as

(ipTSSlope as

IGPParameter3).Value.SetAsText(ipRow.get_Value(ipRow.Fields.FindField("TS_Slope" )).ToString());

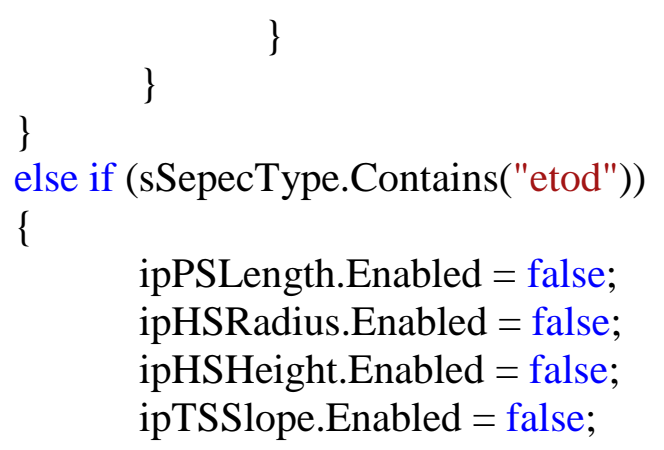

// Get the cusor from the table and loop

through all the rows and find the right specification

m_ipOISSpecTable.Search(null, false);

ICursor ipCursor $=$

IRow ipRow $=$ ipCursor.NextRow();

while (ipRow != null)

\{

if

(ipRow.get_Value(ipRow.Fields.FindField("Type")).ToString().ToLower().Contains("eto d"))

break; 


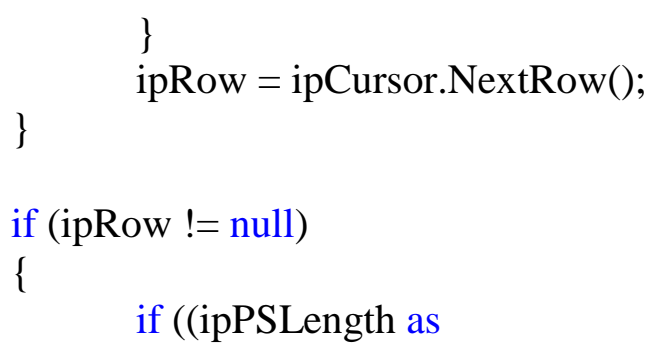

IGPParameter3).Value.SetAsText(ipRow.get_Value(ipRow.Fields.FindField("PS_Length ")). ToString());

IGPParameter3).Altered == false)

\}

if ((ipPSWidth as

\{

IGPParameter3).Value.SetAsText(ipRow.get_Value(ipRow.Fields.FindField("PS_Width ")).ToString());

IGPParameter3).Altered == false)

\} if ((ipHSRadius as

\{

IGPParameter3).Value.SetAsText(ipRow.get_Value(ipRow.Fields.FindField("HS_Radiu s")).ToString());

IGPParameter3).Altered == false)

\}

if ((ipHSHeight as

\{

(ipHSHeight as

IGPParameter3).Value.SetAsText(ipRow.get_Value(ipRow.Fields.FindField("HS_Heigh t")).ToString());

IGPParameter3).Altered == false)

\} if ((ipCSSlope as

\{ (ipCSSlope as

IGPParameter3).Value.SetAsText(ipRow.get_Value(ipRow.Fields.FindField("CS_Slope" )).ToString());

IGPParameter3).Altered == false)

\}

if ((ipCSOffset as 
(ipCSOffset as

IGPParameter3).Value.SetAsText(ipRow.get_Value(ipRow.Fields.FindField("CS_Offset ")).ToString());

IGPParameter3).Altered == false)

\}

if ((ipASLength as

\{

IGPParameter3).Value.SetAsText(ipRow.get_Value(ipRow.Fields.FindField("AS_Lengt h")).ToString());

IGPParameter3).Altered == false)

\}

if ((ipASSlope as

\{ (ipASSlope as

IGPParameter3).Value.SetAsText(ipRow.get_Value(ipRow.Fields.FindField("AS_Slope ")).ToString());

IGPParameter3).Altered == false)

\}

if ((ipASExtendedWidth as

\{

(ipASExtendedWidth as

IGPParameter3).Value.SetAsText(ipRow.get_Value(ipRow.Fields.FindField("AS_Exten dedWidth")).ToString());

IGPParameter3).Altered == false)

\}

if ((ipTSSlope as

\{

(ipTSSlope as

IGPParameter3).Value.SetAsText(ipRow.get_Value(ipRow.Fields.FindField("TS_Slope" )).ToString());

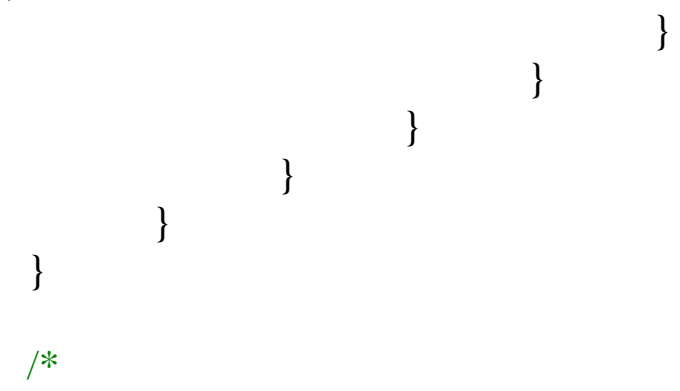

Called after returning from the update parameters routine.

You can examine the messages created from internal validation and

change them if desired.

$* /$

public void UpdateMessages(IArray paramvalues, IGPEnvironmentManager pEnvMgr, IGPMessages Messages)

\{ 
return;

\}

public IGPMessages Validate(IArray paramvalues, bool updateValues, IGPEnvironmentManager envMgr)

\{

return null;

\}

// Execute: Execute the function given the array of the parameters

public void Execute(IArray paramvalues, ITrackCancel trackcancel,

IGPEnvironmentManager envMgr, IGPMessages message)

\{

try

\{

IGPParameter3 parameter;

OISInputParameter oOISInputParameter $=$ new

OISInputParameter();

\#region GeoDatabase

$/ /$ parameter $=$

(IGPParameter)paramvalues.get_Element(m_ciInputDatabaseIndex);

parameter $=($ IGPParameter 3$)$ paramvalues.get_Element $(0)$;

IGPValue ipDatabaseParameterValue =

m_iGPUtilities.UnpackGPValue(parameter);

IDEWorkspace ipDeWorkspace $=$ ipDatabaseParameterValue as

IDEWorkspace;

IDataset ipDataset $=$ m_ipDeUtilites.OpenDataset $($ ipDeWorkspace as IDataElement);

m_ipWorkspace $=$ ipDataset as IWorkspace;

if (m_ipWorkspace != null)

\{

oOISInputParameter.InputDatabasePath =

m_ipWorkspace.PathName;

\#endregion

\#region Input Feature Class

IFeatureClass ipInFeatClass;

parameter $=($ IGPParameter3 $)$ paramvalues.get_Element $(1)$;

m_iGPUtilities.UnpackGPValue(parameter);

IGPValue ipInFeatureClass Value $=$

IQueryFilter QF;

out QF);

m_iGPUtilities.DecodeFeatureLayer(ipInFeatureClassValue, out ipInFeatClass, 
class.");

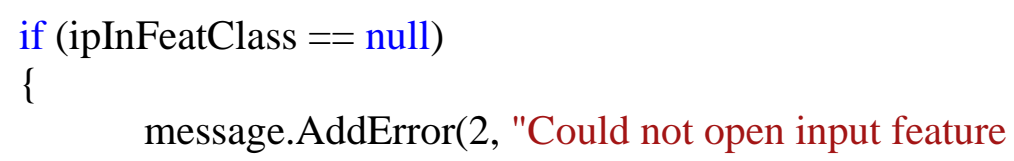

ipInFeatClass;

\}

\#endregion

\#region Output Feature Class

IFeatureClass ipOutFeatClass;

parameter $=($ IGPParameter3 $)$ paramvalues.get_Element $(2)$;

m_iGPUtilities.UnpackGPValue(parameter);

IGPValue ipOutFeatureClass Value =

IQueryFilter outQF;

m_iGPUtilities.DecodeFeatureLayer(ipOutFeatureClassValue, out

ipOutFeatClass, out outQF);

if (ipOutFeatClass $==$ null)

\{

message.AddError(2, "Could not open output

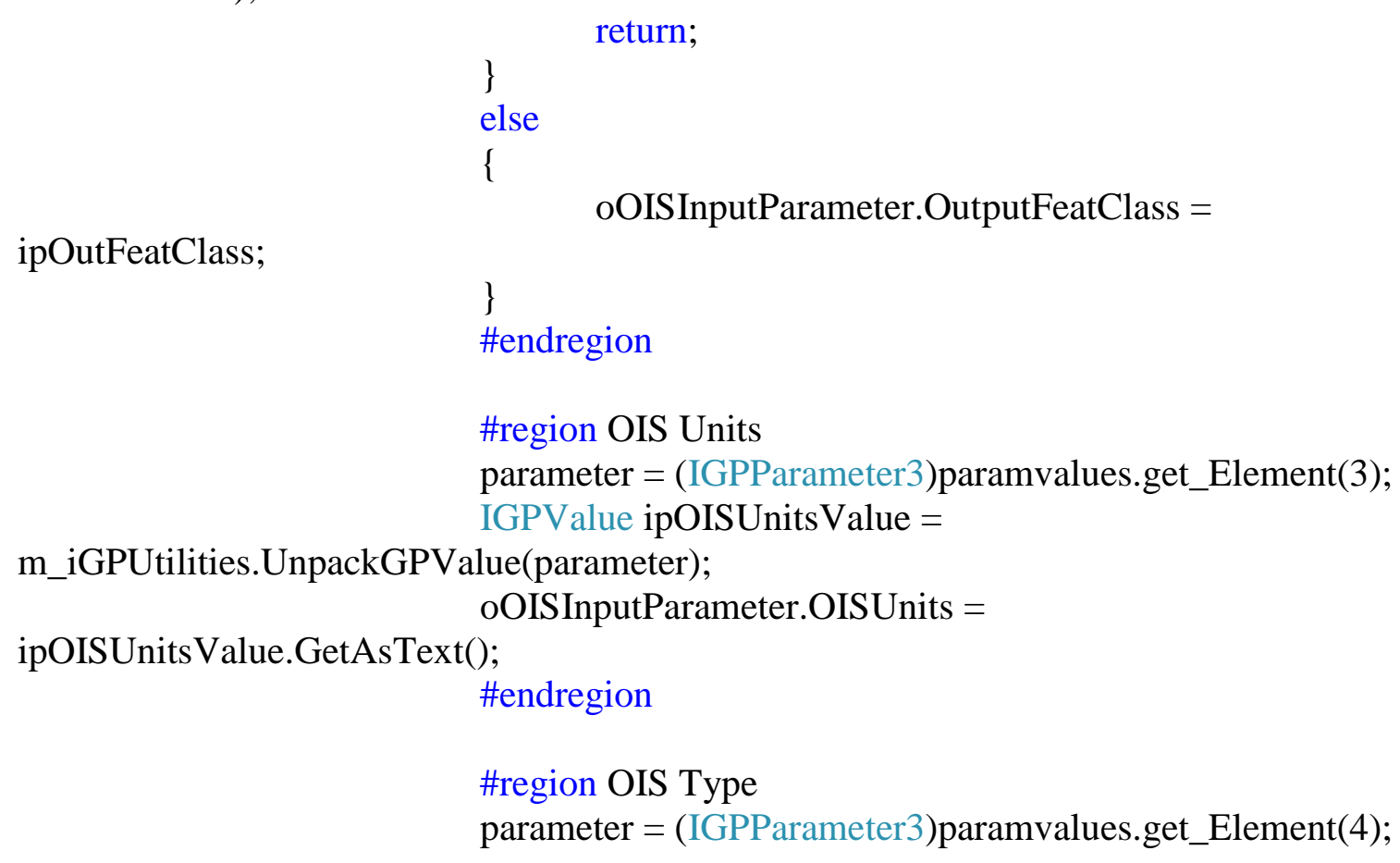


IGPValue ipOISTypeValue $=$

m_iGPUtilities.UnpackGPValue(parameter);

oOISInputParameter.OISType $=$

ipOISTypeValue.GetAsText();

message.AddMessage("OIS are generating by using " +

ipOISTypeValue.GetAsText() + " specification");

\#endregion

\#region Primary Surfaces

parameter $=($ IGPParameter3) $)$ aramvalues.get_Element(5);

m_iGPUtilities.UnpackGPValue(parameter);

IGPValue ipPSLengthValue $=$

oOISInputParameter.PrimarySurfaceLength $=$

(ipPSLengthValue as IGPDouble).Value;

parameter $=($ IGPParameter3 $)$ paramvalues.get_Element $(6)$;

m_iGPUtilities.UnpackGPValue(parameter);

IGPValue ipPSWidthValue $=$

(ipPSWidthValue as IGPDouble).Value;

\#endregion

\#region Horizontal Surface

parameter $=($ IGPParameter3) $)$ aramvalues.get_Element(7);

m_iGPUtilities.UnpackGPValue(parameter);

IGPValue ipHSRadiusValue $=$

(ipHSRadiusValue as IGPDouble).Value;

oOISInputParameter. HorizontalSurfaceRadius =

m_iGPUtilities.UnpackGPValue(parameter);

parameter $=($ IGPParameter3)paramvalues.get_Element $(8)$;

IGPValue ipHSHeightValue $=$

(ipHSHeightValue as IGPDouble).Value;

\#endregion

\#region Conical Surface

parameter $=($ IGPParameter3) $)$ paramvalues.get_Element(9);

IGPValue ipCSSlopeValue $=$

m_iGPUtilities.UnpackGPValue(parameter);

oOISInputParameter. ConicalSurfaceSlope $=$

(ipCSSlopeValue as IGPDouble).Value;

parameter $=$

(IGPParameter3)paramvalues.get_Element(10); 
IGPValue ipCSOffsetValue =

m_iGPUtilities.UnpackGPValue(parameter);

oOISInputParameter.ConicalSurfaceOffset $=$

(ipCSOffsetValue as IGPDouble).Value;

\#endregion

\#region Approach Surface

parameter $=$

(IGPParameter3)paramvalues.get_Element(11);

IGPValue ipAPLengthValue $=$

m_iGPUtilities.UnpackGPValue(parameter);

oOISInputParameter.ApproachSurfaceLength $=$

(ipAPLengthValue as IGPDouble).Value;

parameter $=$

(IGPParameter3)paramvalues.get_Element(12);

IGPValue ipAPSlopeValue $=$

m_iGPUtilities.UnpackGPValue(parameter);

oOISInputParameter. ApproachSurfaceSlope $=$

(ipAPSlopeValue as IGPDouble).Value;

parameter $=$

(IGPParameter3)paramvalues.get_Element(13);

IGPV alue ipAPExtendedWidth Value $=$

m_iGPUtilities.UnpackGPValue(parameter);

oOISInputParameter. ApproachSurfaceExtendedWidth =

(ipAPExtendedWidthValue as IGPDouble).Value;

\#endregion

\#region Transitional Surface

parameter $=$

(IGPParameter3)paramvalues.get_Element(14);

IGPValue ipTSSlopeValue $=$

m_iGPUtilities.UnpackGPValue(parameter);

oOISInputParameter.TransitionalSurfaceSlope $=$

(ipTSSlopeValue as IGPDouble).Value;

\#endregion

OISCreation(oOISInputParameter);

OISCreation oOISCreation $=$ new

bool blncheck = true;

blncheck = oOISCreation.execute(message);

\}

catch (Exception ex)

\{

MessageBox.Show(ex.Message); 


\section{\}}

// This is the function name object for the Geoprocessing Function Tool.

// This name object is created and returned by the Function Factory.

// The Function Factory must first be created before implementing this property. public IName FullName

\{

get

\{

// Add CalculateArea.FullName getter implementation

IGPFunctionFactory functionFactory = new OISGPFunctionFactory();

\} return (IName)functionFactory.GetFunctionName(m_ToolName);

\}

// This is used to set a custom renderer for the output of the Function Tool.

public object GetRenderer(IGPParameter pParam)

\{

return null;

\}

// This is the unique context identifier in a [MAP] file (.h).

// ESRI Knowledge Base article \#27680 provides more information about creating a [MAP] file.

public int HelpContext

\{

get $\{$ return $0 ;\}$

\}

// This is the path to a .chm file which is used to describe and explain the function and its operation.

public string HelpFile

\{

\}

get $\{$ return " "; $\}$

// This is used to return whether the function tool is licensed to execute.

public bool IsLicensed()

\{

return true;

\}

// This is the name of the (.xml) file containing the default metadata for this function tool. 
// The metadata file is used to supply the parameter descriptions in the help panel in the dialog.

// If no (.chm) file is provided, the help is based on the metadata file.

// ESRI Knowledge Base article \#27000 provides more information about creating a metadata file.

public string MetadataFile

\{

\}

get $\{$ return m_metadatafile; $\}$

// This is the class id used to override the default dialog for a tool.

// By default, the Toolbox will create a dialog based upon the parameters returned

// by the ParameterInfo property.

public UID DialogCLSID

\{

\}

get $\{$ return null; $\}$

\#endregion

\} 
private string m_ToolName = "OIS"; $\quad$ // GP Function Name private string $\mathrm{m} \_$metadatafile $=$" ";

private IGPUtilities m_iGPUtilities; // GP Utilities object private IDEUtilities m_ipDeUtilites = new DEUtilitiesClass(); private IWorkspace m_ipWorkspace;

private IFeatureWorkspace m_ipFeatureWorkspace; private ITable m_ipOISSpecTable;

public string m_strDBType = null;

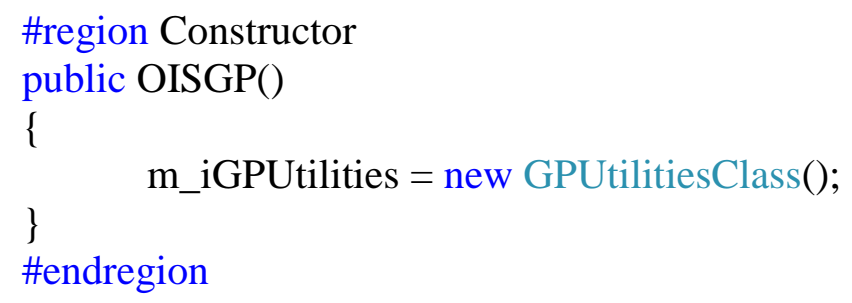

// Set the name of the function tool.

// This name appears when executing the tool at the command line or in scripting. // This name should be unique to each toolbox and must not contain spaces. public string Name

// Set the function tool Display Name as seen in ArcToolbox. public string DisplayName

// This is the location where the parameters to the Function Tool are defined.

// This property returns an IArray of parameter objects (IGPParameter).

// These objects define the characteristics of the input and output parameters. public IArray ParameterInfo

\{

get

\{

IArray iparrParameters = new ArrayClass();

IGPParameterEdit3 ipInputParameter $=$ new

GPParameterClass();

IDEWorkspace ipDEWorkspace $=$ new

DEWorkspaceClass();

ipDEWorkspace. WorkspaceType $=$ esriWorkspaceType.esriLocalDatabaseWorkspace; 
\#region Geodatabase

// input Geodatabase

ipInputParameter.DataType $=$ new

DEWorkspaceTypeClass();

ipInputParameter. Value $=($ IGPValue $)$ ipDEWorkspace;

ipInputParameter. Direction =

esriGPParameterDirection.esriGPParameterDirectionInput;

ipInputParameter.DisplayName $=$

ObstructionIdentificationSurface.OISResource.strDispDB;

ipInputParameter.Enabled = true;

ipInputParameter.Name = "input_Geodatabase";

ipInputParameter.ParameterType $=$

esriGPParameterType.esriGPParameterTypeRequired;

iparrParameters.Add(ipInputParameter);

\#endregion

\#region Input Feature Class

//Input DataType is GPFeatureLayerType

ipInputParameter $=$ new GPParameterClass();

ipInputParameter.DataType $=$ new

GPFeatureLayerTypeClass();

// Default Value object is GPFeatureLayer

ipInputParameter.Value = new GPFeatureLayerClass();

// Set Input Parameter properties

ipInputParameter.Direction $=$

esriGPParameterDirection.esriGPParameterDirectionInput;

ipInputParameter.DisplayName =

ObstructionIdentificationSurface.OISResource.strDispFeatureClass;

ipInputParameter.Enabled = true;

ipInputParameter.Name = "input_features";

ipInputParameter.ParameterType $=$

esriGPParameterType.esriGPParameterTypeRequired;

\#endregion

iparrParameters.Add(ipInputParameter);

\#region Output Feature Class

//Input DataType is GPFeatureLayerType

ipInputParameter $=$ new GPParameterClass();

GPFeatureLayerTypeClass(); ipInputParameter.DataType $=$ new

// Default Value object is GPFeatureLayer

ipInputParameter.Value $=$ new GPFeatureLayerClass();

// Set Input Parameter properties 


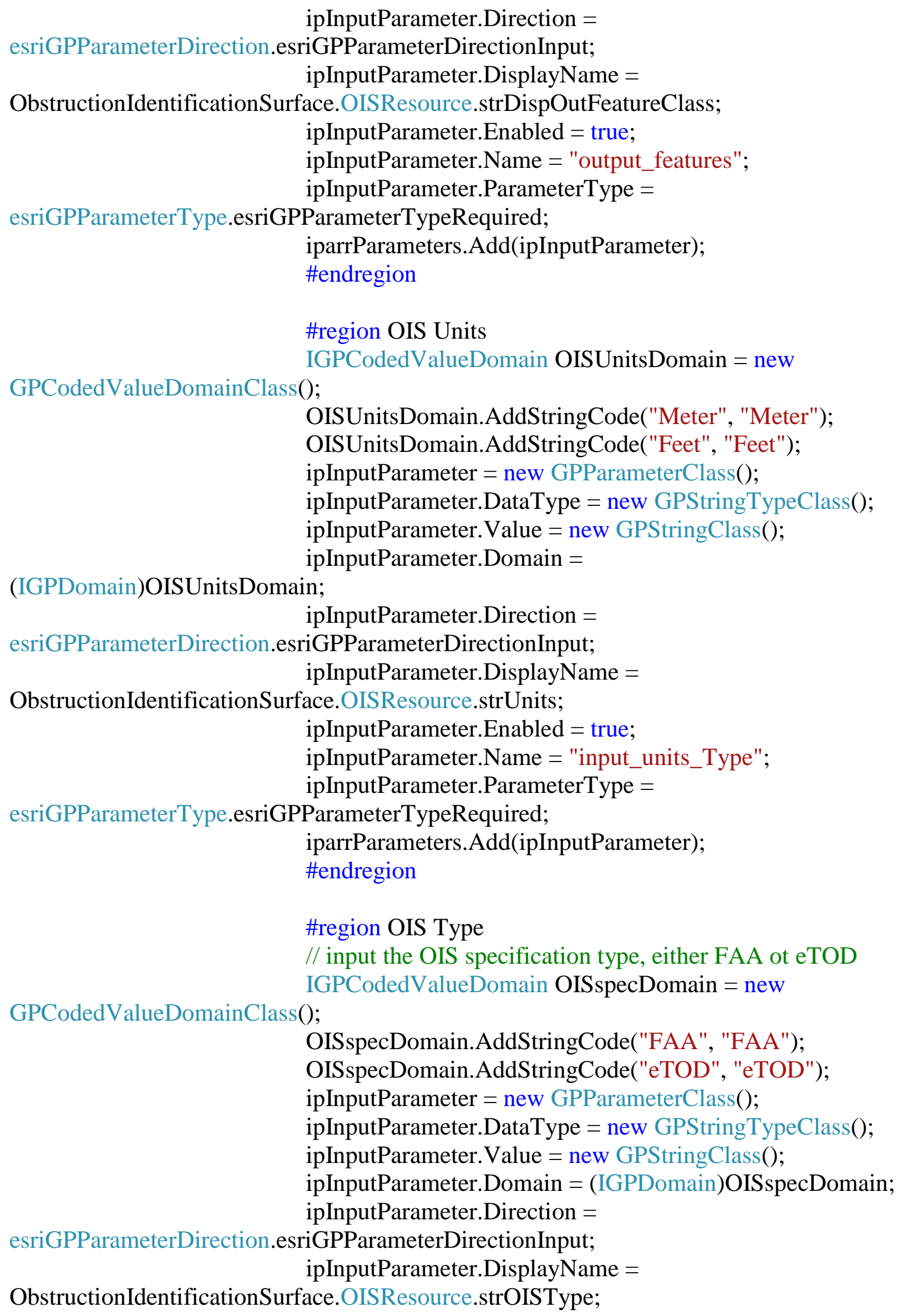


ipInputParameter.Enabled = true;

ipInputParameter.Name = "input_Specification_Type";

ipInputParameter.ParameterType $=$

esriGPParameterType.esriGPParameterTypeRequired;

iparrParameters.Add(ipInputParameter);

\#endregion

\#region Primary Surface

ipInputParameter $=$ new GPParameterClass();

ipInputParameter.DataType = new GPDoubleTypeClass ( $)$;

ipInputParameter.Value = new GPDoubleClass();

ipInputParameter. Direction $=$

esriGPParameterDirection.esriGPParameterDirectionInput;

ipInputParameter.DisplayName $=$

ObstructionIdentificationSurface.OISResource.strOISPSLength;

ipInputParameter.Enabled = true;

ipInputParameter.Name = "input_ps_length";

ipInputParameter.AddDependency("input_Specification_Type");

ipInputParameter.ParameterType =

esriGPParameterType.esriGPParameterTypeRequired;

iparrParameters.Add(ipInputParameter);

ipInputParameter $=$ new GPParameterClass();

ipInputParameter.DataType = new GPDoubleTypeClass();

ipInputParameter.Value = new GPDoubleClass();

ipInputParameter. Direction $=$

esriGPParameterDirection.esriGPParameterDirectionInput;

ipInputParameter.DisplayName =

ObstructionIdentificationSurface.OISResource.strOISPSWidth;

ipInputParameter.Enabled = true;

ipInputParameter.Name = "input_ps_width";

ipInputParameter.AddDependency("input_Specification_Type");

ipInputParameter.ParameterType $=$

esriGPParameterType.esriGPParameterTypeRequired;

iparrParameters.Add(ipInputParameter);

\#endregion

\#region Horizontal Surface

ipInputParameter $=$ new GPParameterClass () ;

ipInputParameter.DataType = new GPDoubleTypeClass();

ipInputParameter. Value $=$ new GPDoubleClass () ;

ipInputParameter.Direction =

esriGPParameterDirection.esriGPParameterDirectionInput; 


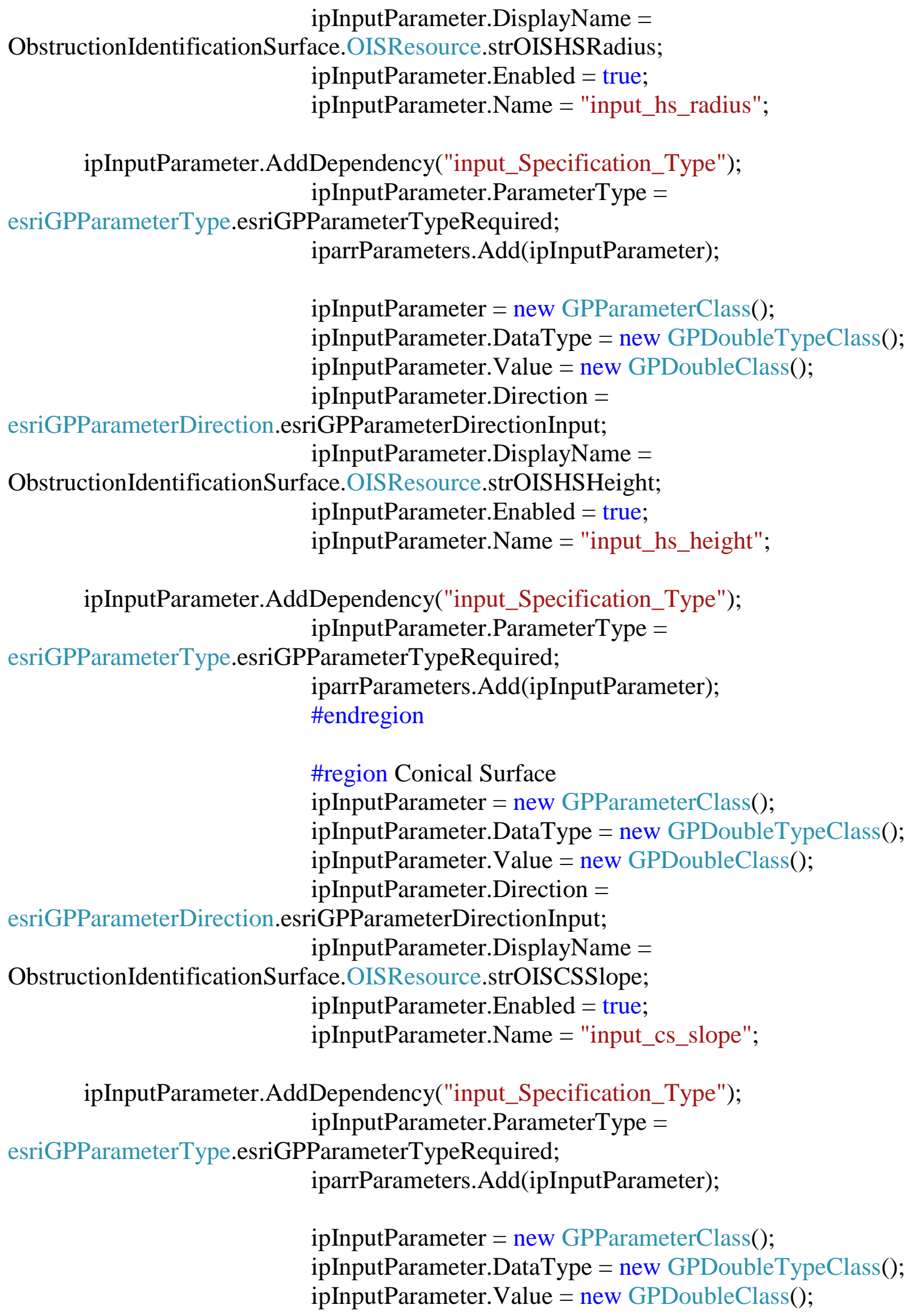




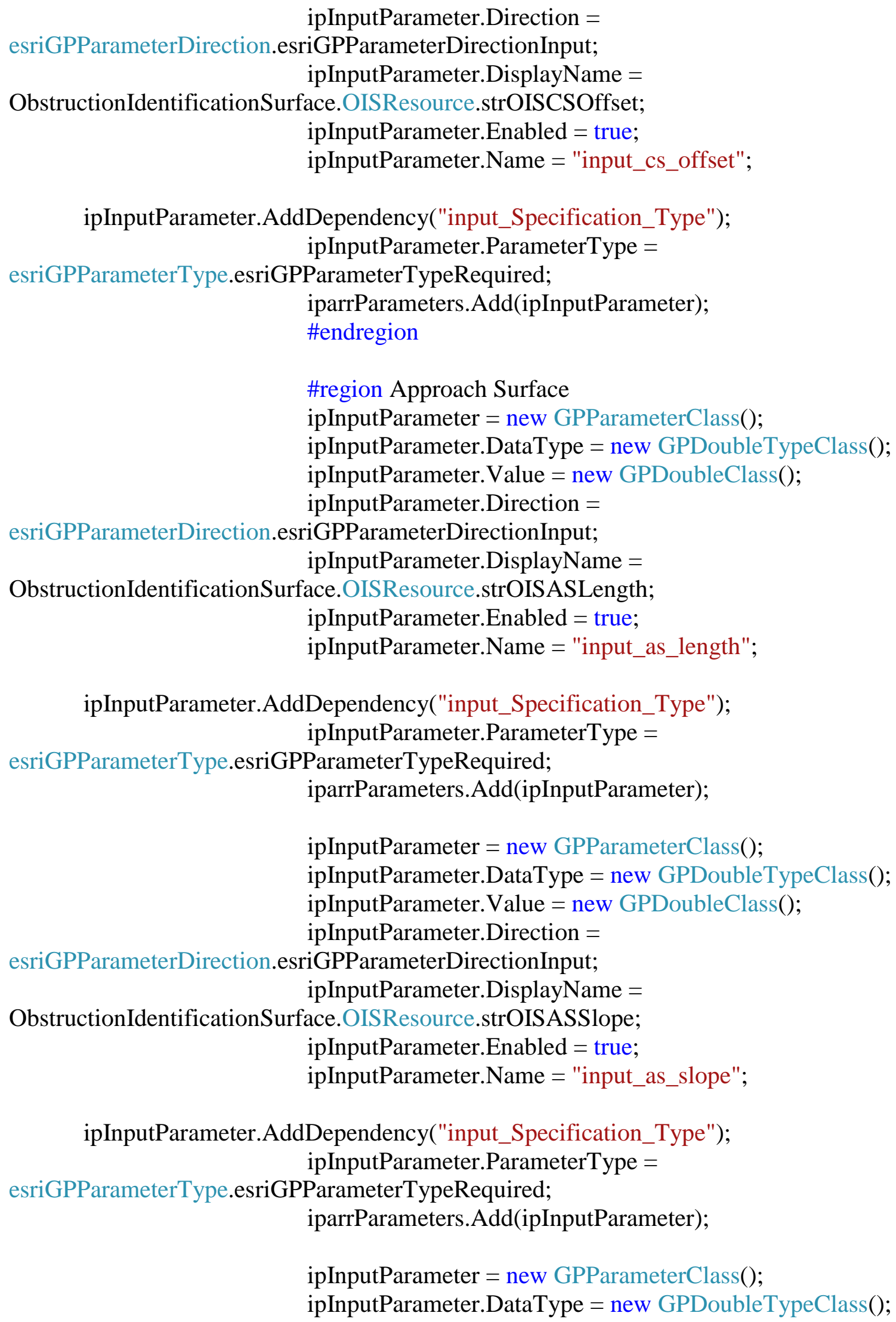




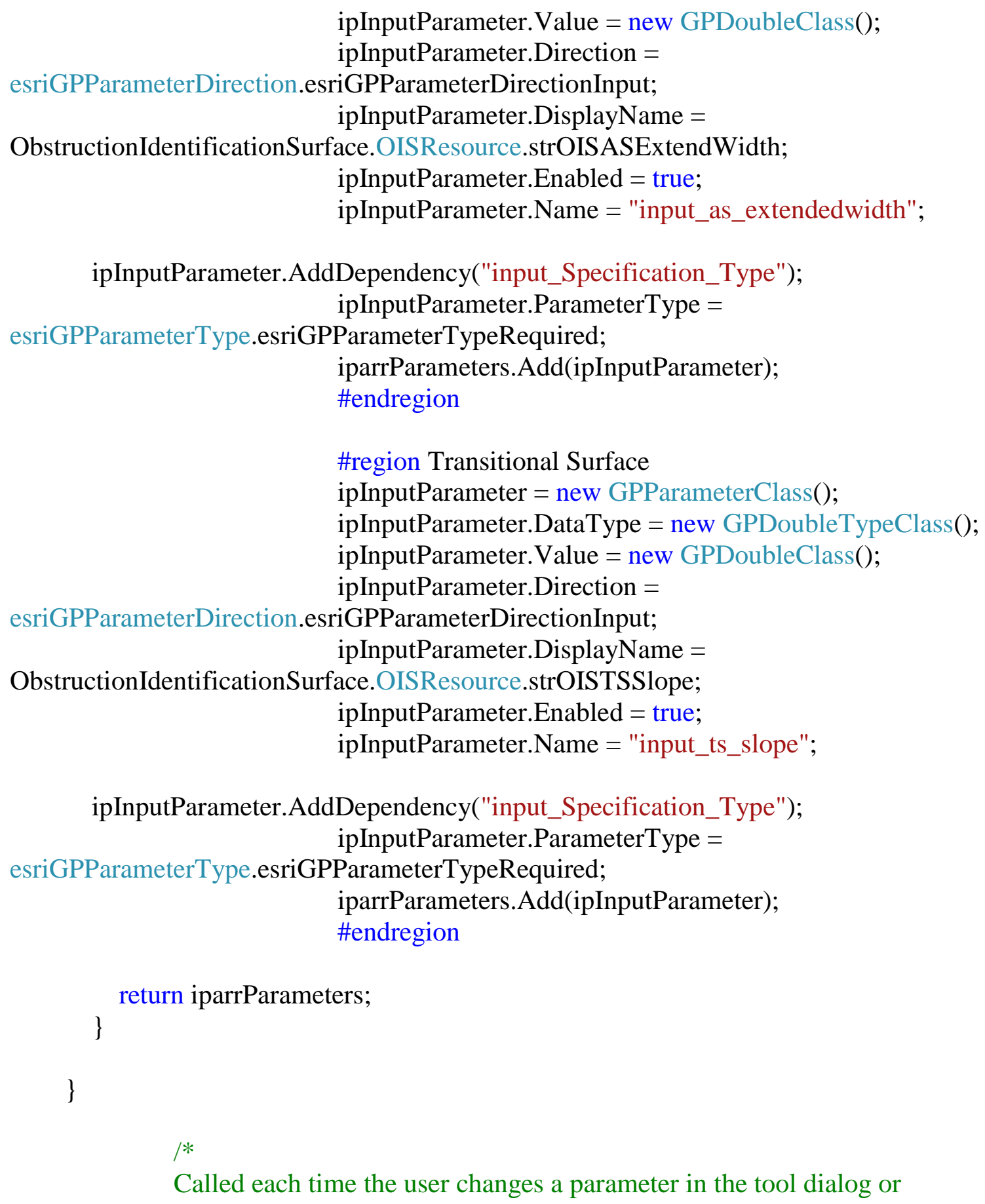

Called each time the user changes a parameter in the tool dialog or building models.

This updates the output data of the tool, which extremely useful for

After returning from UpdateParameters(), geoprocessing calls its internal validation routine checkng that a given set of parameter values

are of the appropriate number, DataType, and value.

This method will update the output parameter value with the unique field. $* /$ 
public void UpdateParameters(IArray paramvalues,

IGPEnvironmentManager pEnvMgr)

IGPParameter3 ipGPDatabase $=$

(IGPParameter3)paramvalues.get_Element(0);

IGPValue ipDatabaseParameterValue $=$

m_iGPUtilities.UnpackGPValue(ipGPDatabase);

IDEWorkspace ipDeWorkspace $=$ ipDatabaseParameterValue as

IDEWorkspace;

as IDataElement);

IDataset ipDataset $=$ m_ipDeUtilites.OpenDataset $(\mathrm{ipDeWorkspace}$

m_ipWorkspace $=$ ipDataset as IWorkspace;

$\mathrm{m} \_$ipFeatureWorkspace $=($IFeatureWorkspace $) \mathrm{m} \_$ipWorkspace;

m_ipOISSpecTable $=$

m_ipFeatureWorkspace.OpenTable("OIS_Spec");

// Retrieve the parameter value

IGPParameterEdit 3 ipSpecParam =

(IGPParameterEdit3)paramvalues.get_Element(4);

IGPParameterEdit 3 ipPSLength $=$

(IGPParameterEdit3)paramvalues.get_Element(5);

IGPParameterEdit3 ipPSWidth $=$

(IGPParameterEdit3)paramvalues.get_Element(6);

IGPParameterEdit 3 ipHSRadius $=$

(IGPParameterEdit3)paramvalues.get_Element(7);

IGPParameterEdit 3 ipHSHeight $=$

(IGPParameterEdit3)paramvalues.get_Element(8);

IGPParameterEdit3 ipCSSlope $=$

(IGPParameterEdit3)paramvalues.get_Element(9);

IGPParameterEdit3 ipCSOffset $=$

(IGPParameterEdit3)paramvalues.get_Element(10);

IGPParameterEdit3 ipASLength =

(IGPParameterEdit3)paramvalues.get_Element(11);

IGPParameterEdit3 ipASSlope $=$

(IGPParameterEdit3)paramvalues.get_Element(12);

IGPParameterEdit 3 ipASExtendedWidth $=$

(IGPParameterEdit3)paramvalues.get_Element(13);

IGPParameterEdit3 ipTSSlope =

(IGPParameterEdit3)paramvalues.get_Element(14);

// If we have an unique field value, create a new field based on the unique field name the user entered.

if ((ipSpecParam as IGPParameter3).Value.IsEmpty ()$==$ false $)$

\{

string sSepecType $=($ ipSpecParam as

IGPParameter3).Value.GetAsText().ToLower(); 
(ipHSRadius as

IGPParameter3).Value.SetAsText(ipRow.get_Value(ipRow.Fields.FindField("HS_Radiu s")).ToString());

IGPParameter3).Altered == false)

if ((ipHSHeight as

$\{\quad$ (ipHSHeight as

IGPParameter3).Value.SetAsText(ipRow.get_Value(ipRow.Fields.FindField("HS_Heigh t")).ToString());

IGPParameter3).Altered == false)

\}

if ((ipCSSlope as

\{

(ipCSSlope as

IGPParameter3).Value.SetAsText(ipRow.get_Value(ipRow.Fields.FindField("CS_Slope" )). ToString());

IGPParameter3).Altered == false)

\}

if ((ipCSOffset as

(ipCSOffset as

IGPParameter3).Value.SetAsText(ipRow.get_Value(ipRow.Fields.FindField("CS_Offset ")). ToString());

IGPParameter3).Altered $==$ false $)$

\}

if ((ipASLength as (ipASLength as

IGPParameter3).Value.SetAsText(ipRow.get_Value(ipRow.Fields.FindField("AS_Lengt h")).ToString());

IGPParameter3).Altered == false)

if ((ipASSlope as

\{

(ipASSlope as

IGPParameter3).Value.SetAsText(ipRow.get_Value(ipRow.Fields.FindField("AS_Slope ")). ToString());

IGPParameter3).Altered $==$ false)

\}

if ((ipASExtendedWidth as

(ipASExtendedWidth as

IGPParameter3).Value.SetAsText(ipRow.get_Value(ipRow.Fields.FindField("AS_Exten dedWidth")).ToString()); 


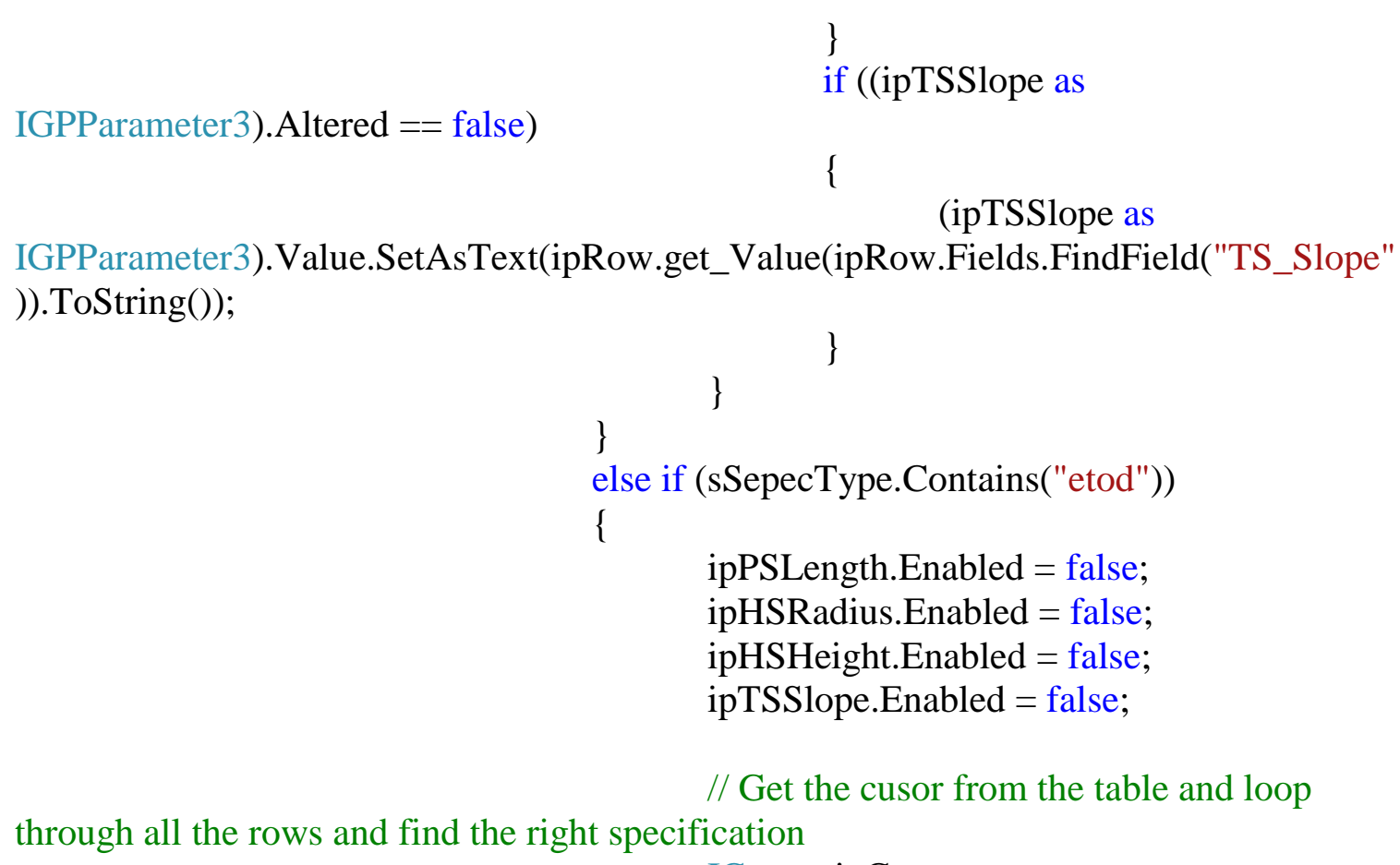

IGPParameter3).Altered == false)

$$
\text { if ((ipTSSlope as }
$$

// Get the cusor from the table and loop

through all the rows and find the right specification

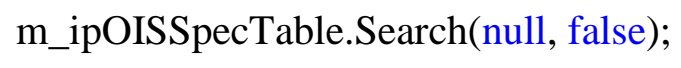

(ipRow.get_Value(ipRow.Fields.FindField("Type")).ToString().ToLower().Contains("eto d"))

IGPParameter3).Altered $==$ false)

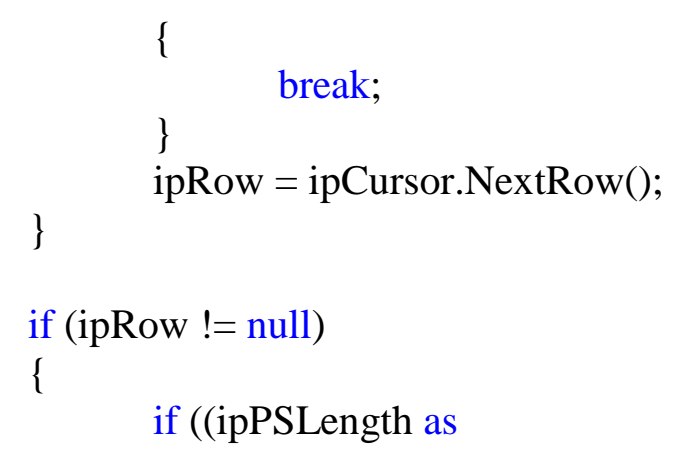

IGPParameter3).Value.SetAsText(ipRow.get_Value(ipRow.Fields.FindField("PS_Length ")).ToString());

IGPParameter3).Altered == false) 
(ipPSWidth as

IGPParameter3).Value.SetAsText(ipRow.get_Value(ipRow.Fields.FindField("PS_Width

")).ToString());

IGPParameter3).Altered $==$ false)

$$
\}
$$

if ((ipHSRadius as

\{

(ipHSRadius as

IGPParameter3).Value.SetAsText(ipRow.get_Value(ipRow.Fields.FindField("HS_Radiu s")).ToString());

IGPParameter3).Altered $==$ false)

\}

if ((ipHSHeight as

\{

(ipHSHeight as

IGPParameter3).Value.SetAsText(ipRow.get_Value(ipRow.Fields.FindField("HS_Heigh

t")).ToString());

IGPParameter3).Altered $==$ false)

if ((ipCSSlope as

(ipCSSlope as

IGPParameter3).Value.SetAsText(ipRow.get_Value(ipRow.Fields.FindField("CS_Slope" )). ToString());

IGPParameter3).Altered == false)

\}

if ((ipCSOffset as

\{

(ipCSOffset as

IGPParameter3).Value.SetAsText(ipRow.get_Value(ipRow.Fields.FindField("CS_Offset

")).ToString());

IGPParameter3).Altered == false)

if ((ipASLength as

(ipASLength as

IGPParameter3).Value.SetAsText(ipRow.get_Value(ipRow.Fields.FindField("AS_Lengt h")).ToString());

IGPParameter3).Altered == false)

if ((ipASSlope as

\{

(ipASSlope as

IGPParameter3).Value.SetAsText(ipRow.get_Value(ipRow.Fields.FindField("AS_Slope ")).ToString()); 
IGPParameter3).Altered == false)

if ((ipASExtendedWidth as

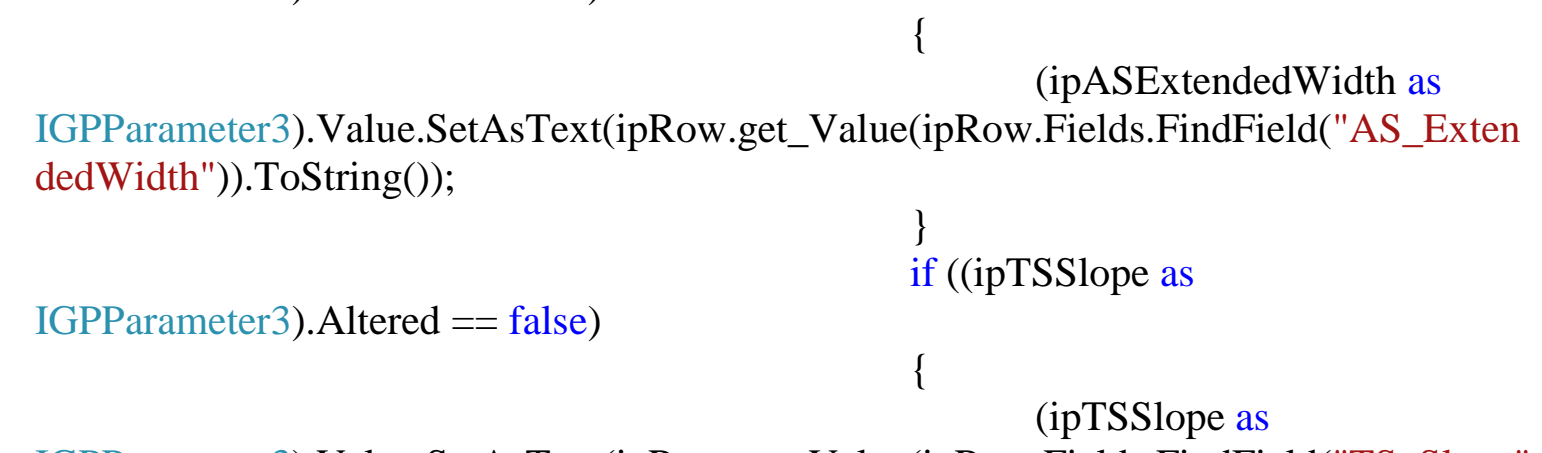

IGPParameter3).Value.SetAsText(ipRow.get_Value(ipRow.Fields.FindField("TS_Slope" )).ToString());

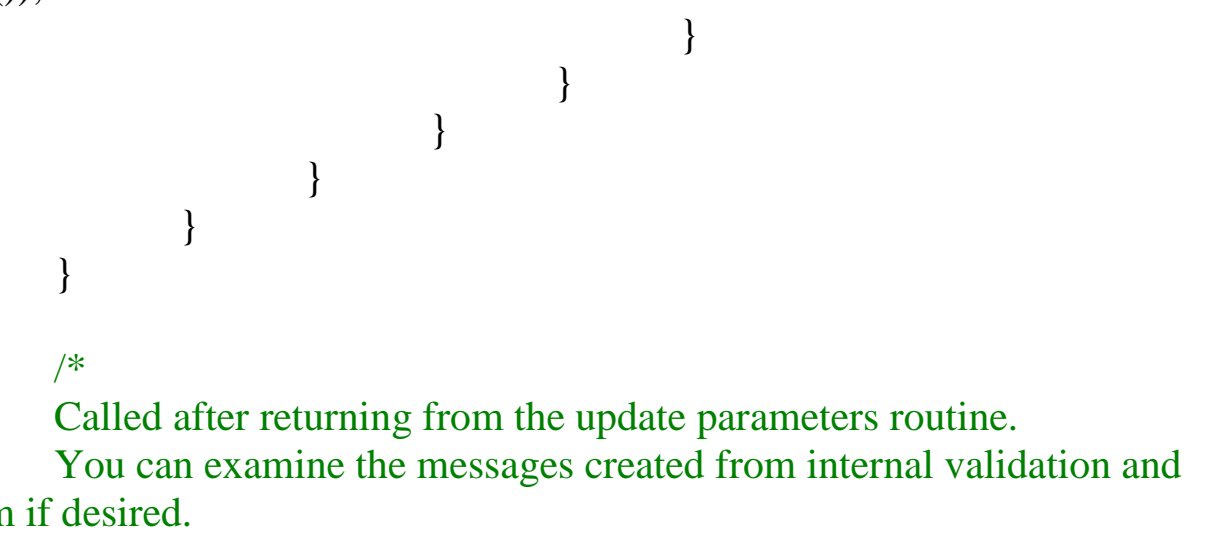

change them if desired.

*/

public void UpdateMessages(IArray paramvalues,

IGPEnvironmentManager pEnvMgr, IGPMessages Messages)

\{

\}

return;

public IGPMessages Validate(IArray paramvalues, bool updateValues, IGPEnvironmentManager envMgr)

\{

return null;

\}

// Execute: Execute the function given the array of the parameters public void Execute(IArray paramvalues, ITrackCancel trackcancel, IGPEnvironmentManager envMgr, IGPMessages message)

\{

try

\{

IGPParameter3 parameter;

OISInputParameter();

OISInputParameter oOISInputParameter $=$ new 
\#region GeoDatabase

$/ /$ parameter $=$

(IGPParameter)paramvalues.get_Element(m_ciInputDatabaseIndex);

parameter $=($ IGPParameter3 $)$ paramvalues.get_Element $(0)$;

IGPValue ipDatabaseParameterValue $=$

m_iGPUtilities.UnpackGPValue(parameter);

IDEWorkspace ipDeWorkspace $=$ ipDatabaseParameterValue as

IDEWorkspace;

IDataset ipDataset $=\mathrm{m} \_$ipDeUtilites.OpenDataset $(\mathrm{ipDeWorkspace}$ as

IDataElement);

m_ipWorkspace $=$ ipDataset as IWorkspace;

if (m_ipWorkspace != null)

\{

m_ipWorkspace.PathName;

oOISInputParameter.InputDatabasePath =

\}

\#endregion

\#region Input Feature Class

IFeatureClass ipInFeatClass;

parameter $=($ IGPParameter3 $)$ paramvalues.get_Element $(1)$;

IGPValue ipInFeatureClass Value =

m_iGPUtilities.UnpackGPValue(parameter);

IQueryFilter QF; out QF);

m_iGPUtilities.DecodeFeatureLayer(ipInFeatureClassValue, out ipInFeatClass,

$$
\text { if (ipInFeatClass }==\text { null) }
$$

\{

class.");

message.AddError(2, "Could not open input feature

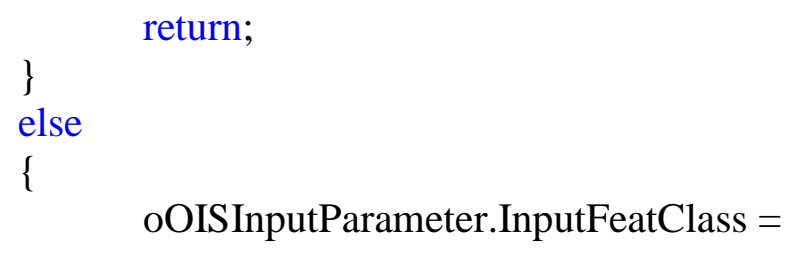

oOISInputParameter.InputFeatClass =

ipInFeatClass;

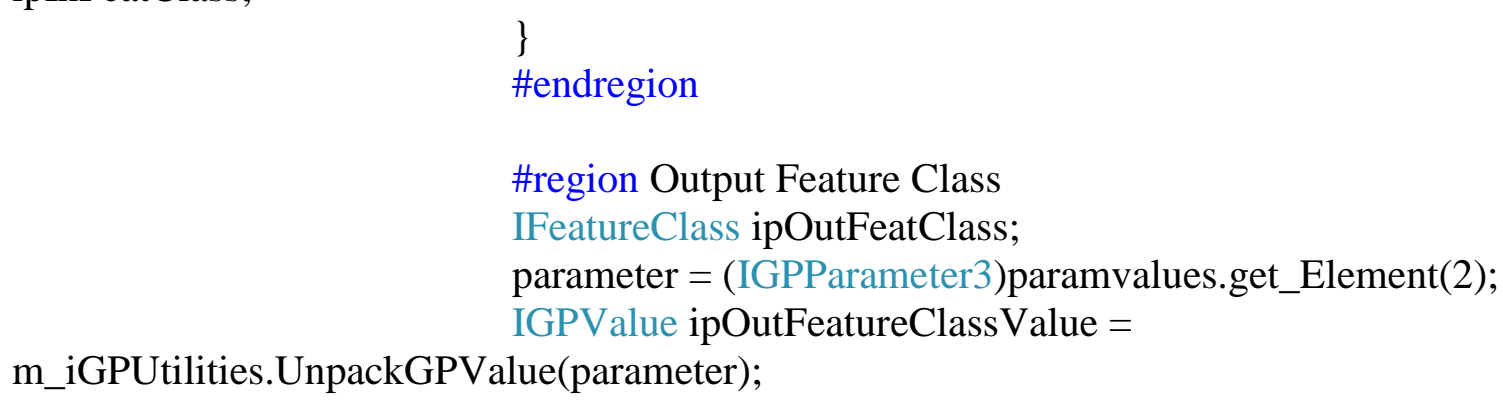




\section{IQueryFilter outQF;}

m_iGPUtilities.DecodeFeatureLayer(ipOutFeatureClassValue, out ipOutFeatClass, out outQF);

if (ipOutFeatClass $==$ null)

\{

feature class.");

message.AddError(2, "Could not open output

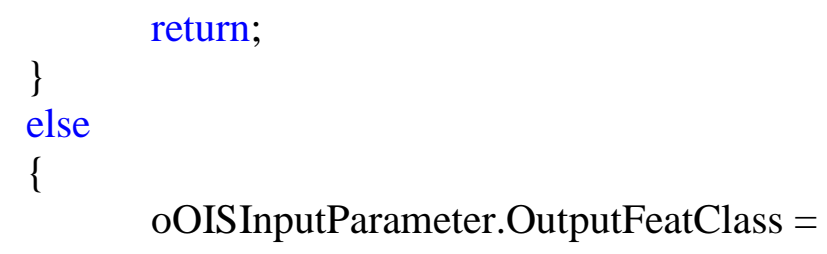

ipOutFeatClass;

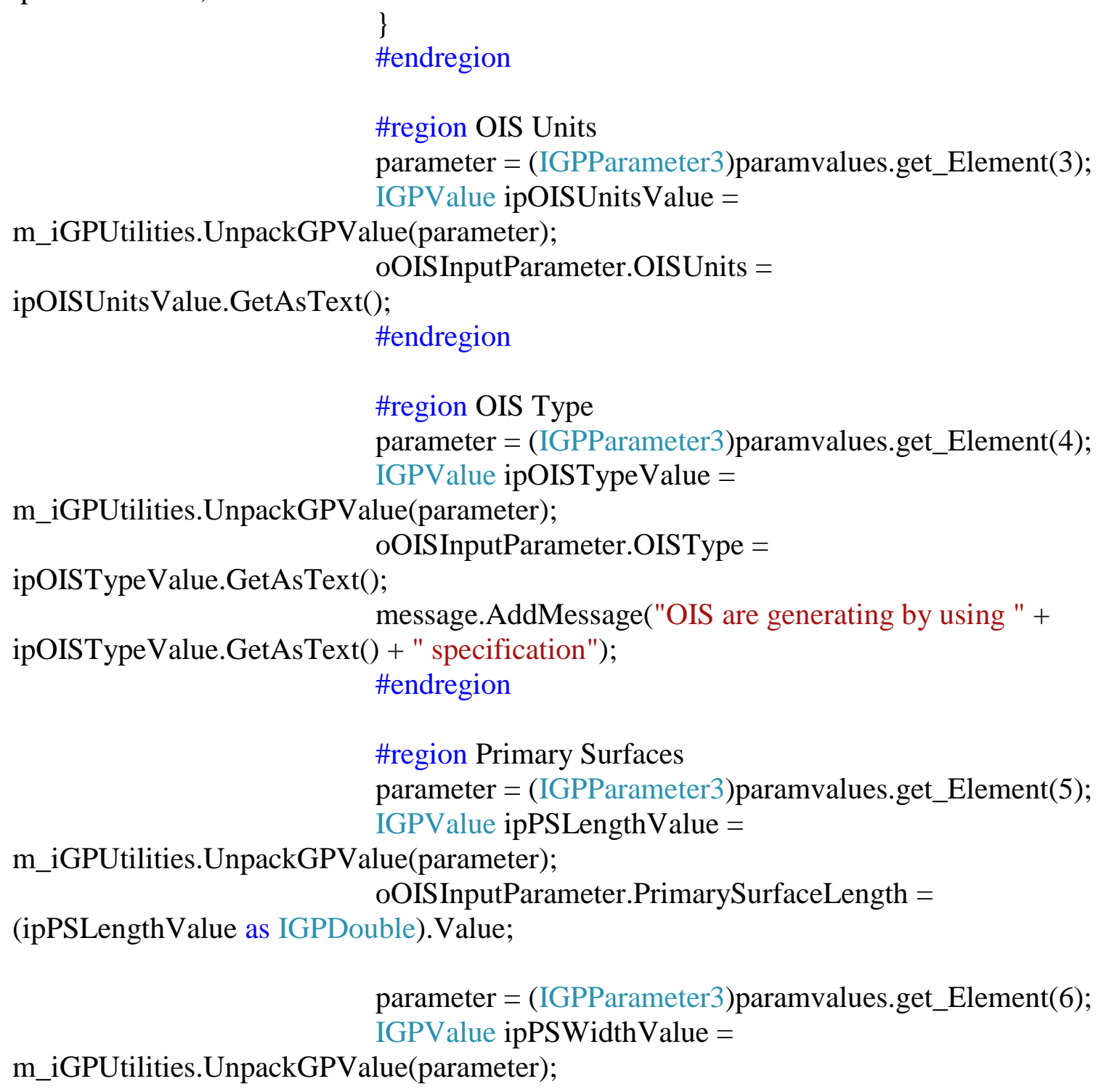


oOISInputParameter.PrimarySurfaceWidth =

(ipPSWidthValue as IGPDouble).Value;

\#endregion

\#region Horizontal Surface

parameter $=($ IGPParameter3) paramvalues.get_Element $(7)$;

m_iGPUtilities.UnpackGPValue(parameter);

IGPValue ipHSRadiusValue $=$ oOISInputParameter.HorizontalSurfaceRadius = (ipHSRadiusValue as IGPDouble).Value;

parameter $=($ IGPParameter3) $)$ paramvalues.get_Element $(8)$;

IGPValue ipHSHeightValue $=$

m_iGPUtilities.UnpackGPValue(parameter); oOISInputParameter .HorizontalSurfaceHeight $=$

(ipHSHeightValue as IGPDouble).Value;

\#endregion

\#region Conical Surface

parameter $=($ IGPParameter3)$)$ paramvalues.get_Element $(9)$;

IGPValue ipCSSlopeValue =

m_iGPUtilities.UnpackGPValue(parameter);

oOISInputParameter. ConicalSurfaceSlope =

(ipCSSlopeValue as IGPDouble).Value;

parameter $=$

(IGPParameter3)paramvalues.get_Element(10);

IGPValue ipCSOffsetValue $=$

m_iGPUtilities.UnpackGPValue(parameter); oOISInputParameter.ConicalSurfaceOffset $=$

(ipCSOffsetValue as IGPDouble).Value;

\#endregion

\#region Approach Surface

parameter $=$

(IGPParameter3)paramvalues.get_Element(11);

IGPValue ipAPLengthValue =

m_iGPUtilities.UnpackGPValue(parameter);

oOISInputParameter. ApproachSurfaceLength $=$

(ipAPLengthValue as IGPDouble).Value;

parameter $=$

(IGPParameter3)paramvalues.get_Element(12);

IGPValue ipAPSlopeValue $=$

m_iGPUtilities.UnpackGPValue(parameter); 


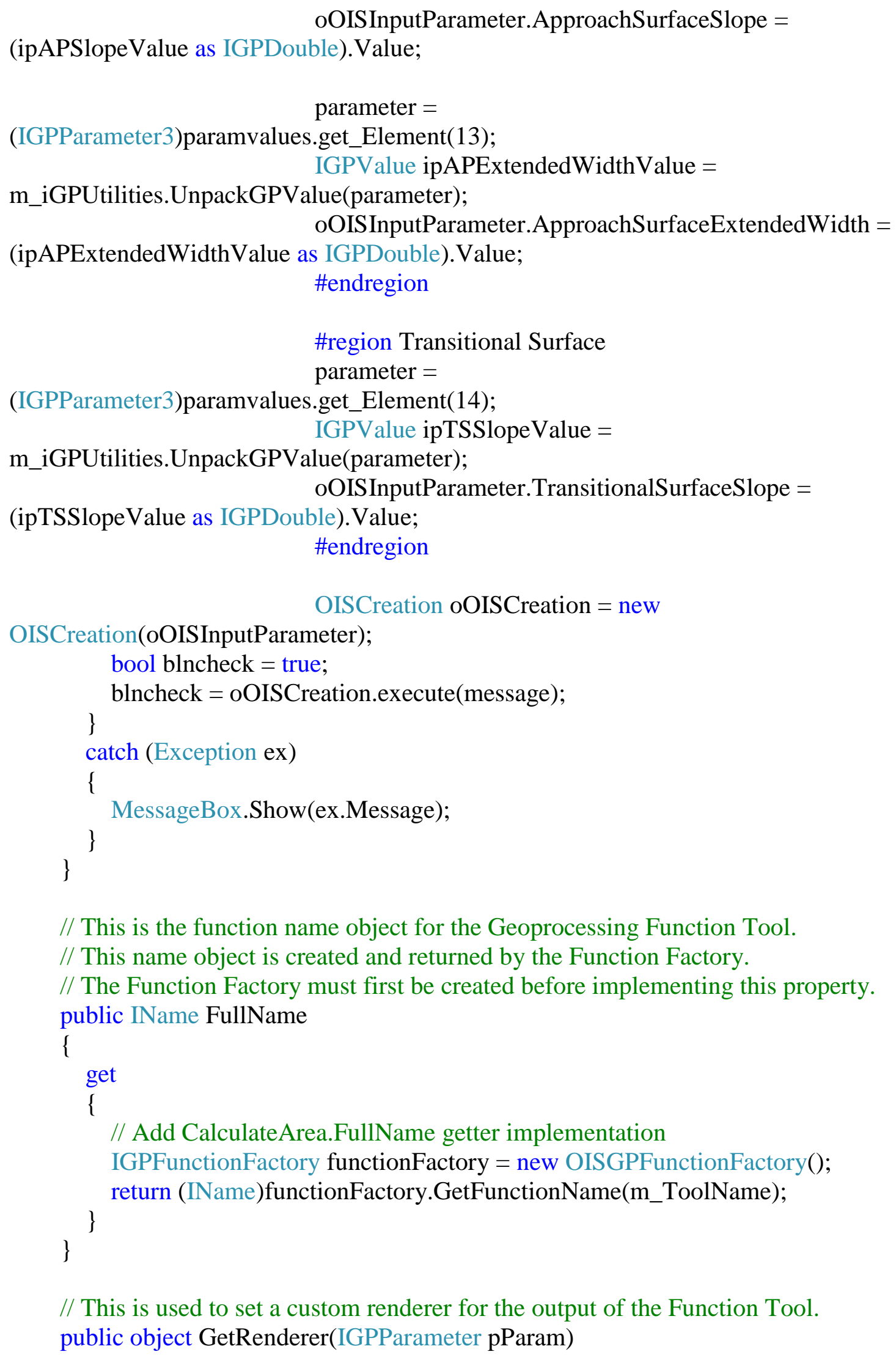

// This is used to set a custom renderer for the output of the Function Tool. public object GetRenderer(IGPParameter pParam) 
\{

return null;

\}

// This is the unique context identifier in a [MAP] file (.h).

// ESRI Knowledge Base article \#27680 provides more information about creating a [MAP] file.

public int HelpContext

\{

get $\{$ return 0 ; $\}$

\}

// This is the path to a .chm file which is used to describe and explain the function and its operation.

public string HelpFile

\{

\}

get $\{$ return " "; $\}$

// This is used to return whether the function tool is licensed to execute.

public bool IsLicensed()

\{

return true;

\}

// This is the name of the (.xml) file containing the default metadata for this function tool.

// The metadata file is used to supply the parameter descriptions in the help panel in the dialog.

// If no (.chm) file is provided, the help is based on the metadata file.

// ESRI Knowledge Base article \#27000 provides more information about creating a metadata file.

public string MetadataFile

\{

\}

get $\left\{\right.$ return $m \_$metadatafile; $\}$

// This is the class id used to override the default dialog for a tool.

// By default, the Toolbox will create a dialog based upon the parameters returned

// by the ParameterInfo property.

public UID DialogCLSID

\{

get $\{$ return null; $\}$

\}

\#endregion 
\}

\} 